\title{
Parameters and Duality for the Metaplectic Geometric Langlands Theory
}

\section{Citation}

Gaitsgory, D., and S. Lysenko. 2018. Parameters and Duality for the Metaplectic Geometric Langlands Theory. Selecta Mathematica 24, no. 1: 227-301.

\section{Permanent link}

http://nrs.harvard.edu/urn-3:HUL.InstRepos:41275771

\section{Terms of Use}

This article was downloaded from Harvard University's DASH repository, and is made available under the terms and conditions applicable to Open Access Policy Articles, as set forth at http:// nrs.harvard.edu/urn-3:HUL.InstRepos:dash.current.terms-of-use\#OAP

\section{Share Your Story}

The Harvard community has made this article openly available.

Please share how this access benefits you. Submit a story.

\section{Accessibility}




\title{
PARAMETERS AND DUALITY FOR THE METAPLECTIC GEOMETRIC LANGLANDS THEORY
}

\author{
D. GAITSGORY AND S. LYSENKO
}

\begin{abstract}
We introduce the space of parameters for the metaplectic Langlands theory as factorization gerbes on the affine Grassmannian, and develop metaplectic Langlands duality in the incarnation of the metaplectic geometric Satake functor.

We formulate a conjecture in the context of the global metaplectic Langlands theory, which is a metaplectic version of the "vanishing theorem" of [Ga5, Theorem 4.5.2].
\end{abstract}

\section{INTRODUCTION}

0.1. What is this paper about? The goal of this paper is to provide a summary of the metaplectic Langlands theory. Our main objectives are:

-Description of the set (rather, space) of parameters for the metaplectic Langlands theory;

-Construction of the metaplectic Langlands dual (see Sect. 0.1.6 for what we mean by this);

-The statement of the metaplectic geometric Satake.

0.1.1. The metaplectic setting. Let $\mathbf{F}$ be a local field and $G$ an algebraic group over $\mathbf{F}$. The classical representation theory of locally compact groups studies (smooth) representations of the group $G(\mathbf{F})$ on vector spaces over another field $E$. Suppose now that we are given a central extension

$$
1 \rightarrow E^{\times} \rightarrow \widetilde{G(\mathbf{F})} \rightarrow G(\mathbf{F}) \rightarrow 1 \text {. }
$$

We can then study representations of $\widetilde{G(\mathbf{F})}$ on which the central $E^{\times}$acts by the tautological character. We will refer to $(0.1)$ as a local metaplectic extension of $G(\mathbf{F})$, and to the above category of representations as metaplectic representations of $G(\mathbf{F})$ corresponding to the extension (0.1).

Let now $\mathbf{F}$ be a global field, and let $\mathbb{A}_{\mathbf{F}}$ be the corresponding ring of adèles. Let us be given a central extension

$$
1 \rightarrow E^{\times} \rightarrow \widetilde{G\left(\mathbb{A}_{\mathbf{F}}\right)} \rightarrow G\left(\mathbb{A}_{\mathbf{F}}\right) \rightarrow 1
$$

equipped with a splitting over $G(\mathbf{F}) \hookrightarrow G\left(\mathbb{A}_{\mathbf{F}}\right)$.

We can then study the space of $E$-valued functions on the quotient $\widetilde{G\left(\mathbb{A}_{\mathbf{F}}\right)} / G(\mathbf{F})$, on which the central $E^{\times}$acts by the tautological character. We will refer to (0.2) as a global metaplectic extension of $G(\mathbf{F})$, and to the above space of functions as metaplectic automorphic functions on $G(\mathbf{F})$ corresponding to the extension (0.2).

There has been a renewed interest in the study of metaplectic representations and metaplectic automorphic functions, e.g., by B.Brubaker-D.Bump-S.Friedberg, P.McNamara, W.T.Gan-F.Gao.

M. Weissman has initiated a program of constructing the L-groups corresponding to metaplectic extensions, to be used in the formulation of the Langlands program in the metaplectic setting, see [We].

Date: September 11, 2017. 
0.1.2. Parameters for metaplectic extensions. In order to construct metaplectic extensions, in both the local and global settings, one starts with a datum of algebro-geometric nature. Namely, one usually takes as an input what we call a Brylinski-Deligne datum, by which we mean a central extension

$$
1 \rightarrow\left(K_{2}\right)_{\text {Zar }} \rightarrow \widetilde{G} \rightarrow G \rightarrow 1,
$$

of sheaves of groups on the big Zariski site of $\mathbf{F}$, where $\left(K_{2}\right)$ Zar is the sheafification of the presheaf of abelian groups that assigns to an affine scheme $S=\operatorname{Spec}(A)$ the group $K_{2}(A)$.

For a local field $\mathbf{F}$, let $\mathbf{f}$ denote its residue field and let us choose a homomorphism

$$
\mathbf{f}^{\times} \rightarrow E^{\times} .
$$

Then taking the group of $\mathbf{F}$-points of $\widetilde{G}$ and pushing out with respect to

$$
K_{2}(\mathbf{F}) \stackrel{\text { symbol }}{\longrightarrow} \mathbf{f}^{\times} \rightarrow E^{\times},
$$

we obtain a central extension (0.1). A similar procedure applies also in the global setting.

0.1.3. The geometric theory. Let $k$ be a ground field and let $G$ be a reductive group over $k$.

In the local geometric Langlands theory one considers the loop group $G((t))$ along with its action on various spaces, such as the affine Grassmannian $\operatorname{Gr}_{G}=G((t)) / G \llbracket t \rrbracket$. Specfically one studies the behavior of categories of sheaves ${ }^{1}$ on such spaces with respect to this action.

In the global geometric Langlands theory one considers a smooth proper curve $X$, and one studies the stack $\operatorname{Bun}_{G}$ that classifies principal $G$-bundles on $X$. The main object of investigation is the category of sheaves on $\operatorname{Bun}_{G}$.

There are multiple ways in which the local and global theories interact. For example, given a ( $k$-rational) point $x \in X$, and identifying the local $\operatorname{ring} \mathcal{O}_{x}$ of $X$ at $x$ with $k \llbracket t \rrbracket$, we have the map

$$
\mathrm{Gr}_{G} \rightarrow \mathrm{Bun}_{G}
$$

where we interpret $\mathrm{Gr}_{G}$ as the moduli space of principal $G$-bundles on $X$, trivialized over $X-x$.

0.1.4. The setting of metaplectic geometric Langlands theory. Let $E$ denote the field of coefficients of the sheaf theory that we consider. Recall (see Sect. 1.7.4) that if $y$ is a space ${ }^{2}$ and $\mathcal{G}$ is a $E^{\times}$-gerbe on $y$, we can twist the category of sheaves on $y$, and obtain a new category, denoted

$$
\operatorname{Shv}_{\mathcal{G}}(y) \text {. }
$$

In the local metaplectic Langlands theory, the input datum (which is an analog of a central extension $(0.1))$ is an $E^{\times}$-gerbe over the loop group $G((t))$ that behaves multiplicaively, i.e., one that is compatible with the group-law on $G((t))$.

Similarly, whenever we consider an action of $G((t))$ on $y$, we equip $y$ with $E^{\times}$-gerbe that is compatible with the given multiplicative gerbe on $G((t))$. In this case we say that the category $\operatorname{Shv}_{\mathcal{G}}(y)$ carries a twisted action of $G((t))$, where the parameter of the twist is our gerbe on $G((t))$.

In the global setting we consider a gerbe $\mathcal{G}$ over $\operatorname{Bun}_{G}$, and the corresponding category $\operatorname{Shv}_{\mathcal{G}}\left(\operatorname{Bun}_{G}\right)$ of twisted sheaves.

Now, if we want to consider the local vs. global interaction, we need a compatibility structure on our gerbes. For example, we need that for every point $x \in X$, the pullback along (0.5) of the given gerbe on $\operatorname{Bun}_{G}$ be a gerbe compatible with some given multiplicative gerbe on $G((t))$.

So, it is natural to seek an algebro-geometric datum, akin to (0.3), that would provide such a compatible family of gerbes.

\footnotetext{
${ }^{1}$ See Sect. 1.1 for what we mean by the category of sheaves.

${ }^{2}$ By a "space" we mean a scheme, stack, ind-scheme, or more generally a prestack, see Sect. 1.1.3 for what the latter word means.
} 
0.1.5. Geometric metaplectic datum. It turns out that such a datum (let us call it "the geometric metaplectic datum") is not difficult to describe, see Sect. 2.4 .1 below. It amounts to the datum of a factorization gerbe with respect to $E^{\times}$on the affine Grassmannian ${ }^{3} \operatorname{Gr}_{G}$ of the group $G$.

In a way, this answer is more elementary than (0.3) in that we are dealing with étale cohomology rather than $K$-theory.

Moreover, in the original metaplectic setting, if the global field $\mathbf{F}$ is the function field corresponding to the curve $X$ over a finite ground field $k$, a geometric metaplectic datum gives rise directly to an extension (0.2).

Finally, a Brylinski-Deligne datum (i.e., an extension (0.3)) and a choice of a character $k^{\times} \rightarrow E^{\times}$ gives rise to a geometric metaplectic datum.

Thus, we could venture into saying that a geometric metaplectic datum is a more economical way, sufficient for most purposes, to encode also the datum needed to set up the classical metaplectic representation/automorphic theory.

0.1.6. The metaplectic Langlands dual. Given a geometric metaplectic datum, i.e., a factorization gerbe $\mathcal{G}$ on $\mathrm{Gr}_{G}$, we attach to it a certain reductive group $H$, a gerbe $\mathcal{G}_{Z_{H}}$ on $X$ with respect to the center $Z_{H}$ of $H$, and a character $\epsilon: \pm 1 \rightarrow Z_{H}$. We refer to the triple

$$
\left(H, \mathcal{G}_{Z_{H}}, \epsilon\right)
$$

as the metaplectic Langlands dual datum corresponding to $\mathcal{G}$.

The datum of $\mathcal{G}_{z_{H}}$ determines the notion of twisted $H$-local system of $X$. Such twisted local systems are supposed to play a role vis-à-vis metaplectic representations/automorphic functions of $G$ parallel to that of usual $\breve{G}$-local systems vis-à-vis usual representations/automorphic functions of $G$.

For example, in the context of the global geometric theory (in the setting of D-modules), we will propose a conjecture (namely, Conjecture 8.6.2) that says that the monoidal category QCoh ( $\operatorname{LocSys}_{H} \mathcal{G}_{Z_{H}}$ ) of quasi-coherent sheaves on the stack $\operatorname{LocSys}{ }_{H}{ }^{Z_{H}}$ classifying such twisted local systems, acts on the category $\operatorname{Shv}_{\mathcal{G}}\left(\operatorname{Bun}_{G}\right)$.

The geometric input for such an action is provided by the metaplectic geometric Satake functor, see Sect. 8.

Presumably, in the arithmetic context, the above notion of twisted $H$-local system coincides with that of homomorphism of the (arithmetic) fundamental group of $X$ to Weissman's L-group.

0.2. "Metaplectic" vs "Quantum". In the paper [Ga4], a program was proposed towards the quantum Langlands theory. Let us comment on the terminological difference between "metaplectic" and "quantum", and how the two theories are supposed to be related.

0.2.1. If $y$ is a scheme (resp., or more generally, a prestack) we can talk about $E^{\times}$-gerbes on it. As was mentioned above, such gerbes on various spaces associated with the group $G$ and the geometry of the curve $X$ are parameters for the metaplectic Langlands theory.

Let us now assume that $k$ has characteristic 0 , and let us work in the context of D-modules. Then, in addition to the notion of $E^{\times}$-gerbe on $y$, there is another one: that of twisting (see Sect. 1.9.5 for what the word "twisting" means).

There is a forgetful map from twistings to gerbes. Roughly speaking, a gerbe $\mathcal{G}$ on $y$ defines the corresponding twisted category of sheaves (=D-modules) $\operatorname{Shv}_{\mathcal{G}}(y)=D-\bmod _{\mathcal{G}}(y)$, while if we lift our gerbe to a twsiting, we also have a forgetful functor

$$
\operatorname{D-mod}_{\mathcal{G}}(y) \rightarrow \mathrm{QCoh}(y) .
$$

\footnotetext{
${ }^{3}$ Here the affine Grassmannian appears in its factorization (a.k.a, Beilinson-Drinfeld) incarnation. I.e., it is a prestack mapping to the Ran space of $X$, rather than $G((t)) / G \llbracket t \rrbracket$, which corresponds to a particular point of $X$.
} 
0.2.2. For the quantum Langlands theory, our parameter will be a factorizable twisting on the affine Grassmannian, which one can also interpret as a Kac-Moody level; we will denote it by $\kappa$.

Thus, for example, in the global quantum geometric Langlands theory, we consider the category

$$
\mathrm{D}-\bmod _{\kappa}\left(\operatorname{Bun}_{G}\right)
$$

which is the same as $\operatorname{Shv}_{\mathcal{G}}\left(\operatorname{Bun}_{G}\right)$, where $\mathcal{G}$ is the gerbe corresponding to $\kappa$.

As was mentioned above, the additional piece of datum that the twisting "buys" us is the forgetful functor

$$
\mathrm{D}-\bmod _{\kappa}\left(\operatorname{Bun}_{G}\right) \rightarrow \mathrm{QCoh}\left(\operatorname{Bun}_{G}\right) \text {. }
$$

In the TQFT interpretation of geometric Langlands, this forgetful functor is called "the big brane". It allows us to relate the category D- $\bmod _{\kappa}\left(\operatorname{Bun}_{G}\right)$ to representations of the Kac-Moody algebra attached to $G$ and the level $\kappa$.

0.2.3. Consider the usual Langlands dual group $\check{G}$ of $G$, and if $\kappa$ is non-degenerate, it gives rise to a twisting, denoted $-\kappa^{-1}$, on the affine Grassmannian $\operatorname{Gr}_{\breve{G}}$ of $\check{G}$.

In the global quantum geometric theory one expects to have an equivalence of categories

$$
\mathrm{D}-\bmod _{\kappa}\left(\operatorname{Bun}_{G}\right) \simeq \mathrm{D}-\bmod _{-\kappa^{-1}}\left(\operatorname{Bun}_{\check{G}}\right)
$$

We refer to (0.6) as the global quantum Langlands equivalence.

0.2.4. How are the two theories related? The relationship between the equivalence (0.6) and the metaplectic Langlands dual is the following:

Let $\mathcal{G}$ (resp., $\check{\mathcal{G}}$ ) be the gerbe on $\operatorname{Gr}_{G}$ (resp., $\mathrm{Gr}_{\breve{G}}$ ) corresponding to $\kappa$ (resp., $-\kappa^{-1}$ ). We conjecture that the metaplectic Langlands dual data $\left(H, \mathcal{G}_{Z_{H}}, \epsilon\right)$ corresponding to $\mathcal{G}$ and $\check{\mathcal{G}}$ are isomorphic.

Furthermore, we conjecture that the resulting actions of

$$
\mathrm{QCoh}\left(\operatorname{LocSys}_{H} \mathfrak{g}_{Z_{H}}\right)
$$

on D- $\bmod _{\kappa}\left(\operatorname{Bun}_{G}\right)$ and D-mod $-_{-\kappa^{-1}}\left(\operatorname{Bun}_{\check{G}}\right)$, respectively (see Sect. 0.1.6 above) are intertwined by the equivalence (0.6).

0.3. What is actually done in this paper? Technically, our focus is on the geometric metaplectic theory, with the goal of constructing the metaplectic geometric Satake functor.

0.3.1. The mathematical content of this paper is the following:

-We define a geometric metaplectic datum to be a factorization gerbe on the (factorization version) of affine Grassmannian $\mathrm{Gr}_{G}$. This is done in Sect. 2.

-We formulate the classification result that describes factorization gerbes on $\mathrm{Gr}_{G}$ in terms of étale cohomology on the classifying stack $B G$ of $G$. This is done in Sect. 3 .

This classification result is inspired by an analogous one in the topological setting, explained to us by J. Lurie.

-We study the relationship between factorization gerbes on $\operatorname{Gr}_{G}$ and those on $\operatorname{Gr}_{M}$, where $M$ is the Levi quotient of a parabolic $P \subset G$. This is done in Sect. 5 .

The main point is that the naive map from factorization gerbes on $\mathrm{Gr}_{G}$ to those on $\mathrm{Gr}_{M}$ needs to be corrected by a gerbe that has to do with signs. It is this correction that is responsible for the fact that the usual geometric Satake does not quite produce the category $\operatorname{Rep}(\check{G})$, but rather its modification where we alter the commutativity constraint by a canonical character $\pm 1 \rightarrow Z(\check{G})$.

-We define the notion of metaplectic Langlands dual datum, denoted $\left(H, \mathcal{G}_{Z_{H}}, \epsilon\right)$, attached to a given geometric metaplectic datum $\mathcal{G}$. We introduce the notion of $\mathcal{G}_{Z_{H}}$-twisted $H$-local system on $X$; when we work with D-modules, these local systems are $k$-points of a (derived) algebraic stack, denoted $\operatorname{LocSys}_{H}{ }^{\mathcal{S}_{Z}}$. This is done in Sect. 6 . 
-We show that a factorization gerbe on $\mathrm{Gr}_{G}$ gives rise to a multiplicative gerbe over the loop group $G((t))$ for every point $x \in X$. Moreover, these multiplicative gerbes also admit a natural factorization structure when instead of a single point $x$ we consider the entire Ran space. This is done in Sect. 7.

-We define metaplectic geometric Satake as a functor between factorization categories over the Ran space.

-We formulate a conjecture about the action of the monoidal category QCoh $\left(\operatorname{LocSys} \mathcal{G}_{H}\right)$ on $\operatorname{Shv}_{\mathcal{G}}\left(\operatorname{Bun}_{G}\right)$. This is done in Sect. 8.

0.3.2. A disclaimer. Although most of the items listed in Sect. 0.3.1 have not appeared in the previously existing literature, this is mainly due to the fact that these earlier sources, specifically the paper [FL] of M. Finkelberg and the second-named author and the paper [Re] of R. Reich, did not use the language of $\infty$-categories, while containing most of the relevant mathematics.

So, one can regard the present paper as a summary of results that are "almost known", but formulated in the language that is better adapted to the modern take on the geometric Langlands theory ${ }^{4}$.

We felt that there was a need for such a summary in order to facilitate further research in this area.

Correspondingly, our focus is on statements, rather than proofs. Most of the omitted proofs can be found in either [FL] or [Re], or can be obtained from other sources cited in the paper.

Below we give some details on the relation of contents of this paper and some of previously existing literature.

0.3.3. Relation to other work: geometric theory. As was just mentioned, a significant part of this paper is devoted to reformulating the results of [FL] and [Re] in a way tailored for the needs of the geometric metaplectic theory.

The paper [Re] develops the theory of factorization gerbes on $\mathrm{Gr}_{G}$ (in loc. cit. they are called "symmetric factorizable gerbes"). One caveat is that in the setting of [Re] one works with schemes over $\mathbb{C}$ and sheaves in the analytic topology, while in the present paper we work over a general ground field and étale sheaves.

The main points of the theory developed in [Re] are the description of the homotopy groups of the space of factorization gerbes (but not of the space itself; the latter is done in Sect. 3 of the present paper), and the fact that a factorization gerbe on $\mathrm{Gr}_{G}$ gives rise to a multiplicative gerbe on (the factorization version of) the loop group (we summarize this construction in Sect. 7 of the present paper).

The proofs of the corresponding results in [Re] are obtained by reducing assertions for a reductive group $G$ to that for its Cartan subgroup, and an explicit analysis for tori. We do not reproduce these proofs in the present paper.

In both $[\mathrm{FL}]$ and $[\mathrm{Re}]$, metaplectic geometric Satake is stated as an equivalence of certain abelian categories. In [FL], this is an equivalence of symmetric monoidal categories (corresponding to a chosen point $x \in X$ ), for a particular class of gerbes (namely, ones obtained from the determinant line bundle).

In $[\mathrm{Re}]$ more general gerbes are considered and the factorization structure on both sides of the equivalence is taken into account. Our version of metaplectic geometric Satake is a statement at the level of DG categories; it is no longer an equivalence, but rather a functor in one direction, between monoidal factorization categories. In this form, our formulation is a simple consequence of that of [Re].

0.3.4. Relation to other work: arithmetic theory. As was already mentioned above, our notion of the metaplectic Langlands dual datum is probably equivalent to the datum constructed by M. Weissman in $[\mathrm{We}]$ for his definition of the L-group.

\footnotetext{
${ }^{4}$ This excludes, however, the material in Sect. 8.5 and the statement of Conjecture 8.6.2 (the latter is new, to the best of our knowledge)
} 
0.4. Structure of the paper. The paper begins with a section that contains some background on prestacks, $\infty$-categories, sheaf theories, etc. The reader who has a rudimentary familiarity with this material can safely skip it.

As has been mentioned already, our geometric metaplectic datum is a gerbe on the affine Grassmannian, denoted $\mathrm{Gr}_{G}$. We will need the factorization (a.k.a. Beilinson-Drinfeld, Ran space) version of the affine Grassmannian. Its key feature is that it is not a scheme, and not even an ind-scheme. Rather, this version of the affine Grassmannian is what we call a prestack. In Sect. 1 we recall the definition of what a prestack is, and what gerbes on a prestack are.

In addition, in the same section we recall what we mean by the category of sheaves on a prestack, and by a sheaf of categories over a prestack. Both these notions are necessary for the statement of metaplectic geometric Satake.

0.4.1. In Sect. 2 we recall the definition of the Ran space of a given curve $X$ (denoted Ran). We define the notions of a factorization prestack over Ran, a factorization gerbe over a factorization prestack and a (pre)factorization sheaf of categories over Ran.

Our main example of a factorization prestack is the affine Grassmannian $\mathrm{Gr}_{G}$. Factorization gerbes over $\operatorname{Gr}_{G}$ are the main object of study in this paper; they provide an input data for the metaplectic geometric Langlands theory.

0.4.2. In Sect. 3 we discuss the parameterization of the set (more precisely, space) of factorization gerbes on $\operatorname{Gr}_{G}$ in terms of étale cohomology of the classifying stack $B G$ of $G$.

We will see that to a factorization gerbe we can associate a combinatorial invariant, denoted $q$, which a quadratic form on the coweight lattice $\Lambda$ of $G$ with coefficients in $E^{\times}$(here $E$ is our field of coefficients), invariant with respect to the Weyl group.

The space of factorization gerbes with a fixed parameter $q$ is of local nature with respect to our curve $X$. More precisely, it is acted on simply transitively by (the commutative group in spaces of) gerbes on $X$ with respect to the group $\operatorname{Hom}\left(\pi_{1, \text { alg }}(G), E^{\times}\right)$.

0.4.3. In Sect. 4 we make our analysis of factorization gerbes on $\operatorname{Gr}_{G}$ more explicit in the case when $G$ is a torus. In fact, most of proofs of statements left unproved in this paper go by reduction to this case.

0.4.4. In Sect. 5 we study the interaction between factorization gerbes on $\operatorname{Gr}_{G}$ and those on $\operatorname{Gr}_{M}$, where $M$ is the Levi quotient of a parabolic $P \subset G$.

The two affine Grassmannians are related by the diagram

$$
\mathrm{Gr}_{G} \stackrel{\mathfrak{p}}{\longleftarrow} \operatorname{Gr}_{P} \stackrel{\mathfrak{q}}{\longrightarrow} \mathrm{Gr}_{M}
$$

where the map $\mathfrak{q}$ has contractible fibers. Hence, given a gerbe on $\mathrm{Gr}_{G}$, we can restrict it to $\mathrm{Gr}_{P}$, and the resulting gerbe will uniquely come from a gerbe on $\mathrm{Gr}_{M}$.

This procedure gives a map from the space of gerbes $\mathrm{Gr}_{G}$ to that on $\mathrm{Gr}_{M}$. However, this map is not quite what we want. Namely, it differs from the "right" one by a certain gerbe that has to do with signs.

0.4.5. In Sect. 6 we explain how the datum of a factorization gerbe $\mathcal{G}$ on $\operatorname{Gr}_{G}$ gives rise to a metaplectic Langlands dual datum, i.e., a reductive group $H$, a gerbe $\mathcal{G}_{Z_{H}}$ on $X$ with respect to the center $Z_{H}$ of $H$, and a character $\pm 1 \rightarrow Z_{H}$.

We define the notion of $\mathcal{G}_{Z_{H}}$-twisted local system on $X$, and when we work with D-modules over a field of characteristic 0 , we construct the (derived) algebraic stack LocSys ${ }_{H}{ }_{Z_{H}}$ that classifies such local systems. 
0.4.6. In Sect. 7 we introduce (the factorization version of) the loop group, denoted $\mathfrak{L}(G)$. We explain a construction that, starting from a factorization gerbe $\mathcal{G}$ on $\mathrm{Gr}_{G}$, produces a multiplicative structure on the pullback of $\mathcal{G}$ to $\mathfrak{L}(G)$. This multiplicative structure is what allows us to define the convolution product on the metaplectic spherical Hecke category.

0.4.7. In Sect. 8 we state the existence of the metaplectic geometric Satake functor, which maps the factorization category, built out of $\left(\operatorname{Rep}(H), \mathcal{G}_{Z_{H}}, \epsilon\right)$ (here $\operatorname{Rep}(H)$ denotes the category of representations of $H$ ), to the metaplectic spherical Hecke category.

Finally, we state our "metaplectic vanishing conjecture", Conjecture 8.6.2 about the action of QCoh $\left(\operatorname{LocSys}_{H}^{\mathcal{I}_{Z}}\right)$ on $\operatorname{Shv}_{\mathcal{G}}\left(\operatorname{Bun}_{G}\right)$

\subsection{Conventions.}

0.5.1. Algebraic geometry. In the main body of the paper we will be working over a fixed ground field $k$, assumed algebraically closed.

For arithmetic applications one would also be interested in the case of $k$ being a finite field $\mathbb{F}_{q}$. However, since all the constructions in this paper are canonical, the results over $\mathbb{F}_{q}$ can be deduced from those over $\overline{\mathbb{F}}_{q}$ by Galois descent.

We will denote by $X$ a smooth connected algebraic curve over $k$ (we do not need $X$ to be complete).

For the purposes of this paper, we do not need derived algebraic geometry, with the exception of Sects. 6.6 and 8.6 (which are devoted to the D-module situation).

We let $\mathrm{Sch}^{\text {aff }}$ denote the category of (classical!) affine schemes over $k$, and by $\mathrm{Sch}_{\mathrm{ft}}^{\text {aff }}$ its full subcategory consisting of affine schemes of finite type.

In the main body of the paper we will make an extensive use of algebro-geometric objects more general than schemes, namely, prestacks. We recall the definition of prestacks in Sect. 1.1.3, and refer the reader to [GR2, Chapter 2] for a more detailed discussion.

0.5.2. Coeffients. We will work with various sheaf theories on schemes, see Sect. 1.1.

Our sheaves will have coefficients in $E$-vector spaces, where $E$ is a field of coefficients, assumed algebraically closed and of characteristic zero.

0.5.3. Groups. We will work with a fixed connected algebraic group $G$ over $k$; our main interest is the case when $G$ is reductive.

We will denote by $\Lambda$ the coweight lattice of $G$ and by $\check{\Lambda}$ its dual, i.e., the weight lattice.

We will denote by $\alpha_{i} \in \Lambda$ (resp., $\check{\alpha}_{i} \in \check{\Lambda}$ ) the simple coroots (resp., roots), where $i$ runs over the set of vertices of the Dynkin diagram of $G$.

If $G$ is reductive, we denote by $\check{G}$ its Langlands dual, viewed as a reductive group over $E$.

0.5.4. The usage of higher category theory. Although, as we have said above, we do not need derived algebraic geometry, we do need higher category theory. However, we only really need $\infty$-categories for one type of manipulation: in order to define the notion of the category of sheaves on a given prestack (and a related notion of a sheaf of categories over a prestack); we will recall the corresponding definitions in Sects. 1.1.3 and 1.6), respectively. These definitions involve the procedure of taking the limit, and the language of higher categories is the adequate framework for doing so.

In their turn, sheaves of categories on prestacks appear for us as follows: the metaplectic spherical Hecke category, which is the recipient of the metaplectic geometric Satake functor (and hence is of primary interest for us), is a sheaf of categories over the Ran space. 
0.5.5. Glossary of $\infty$-categories. We will now recall several most common pieces of notation, pertaining to $\infty$-categories, used in this paper. We refer the reader to [Lu1, Lu2] for the foundations of the theory, or [GR2, Chapter 1] for a concise summary.

We denote by Spc the $\infty$-category of spaces. We denote by $*$ the point-space. For a space $\mathcal{S}$, we denote by $\pi_{0}(\mathcal{S})$ its set of connected components. If $\mathcal{S}$ is a space we can view it as an $\infty$-category; its objects are also called the points of $\mathcal{S}$.

For an $\infty$-category $\mathbf{C}$ and two objects $\mathbf{c}_{0}, \mathbf{c}_{1} \in \mathbf{C}$, we let $\operatorname{Maps}_{\mathbf{C}}\left(\mathbf{c}_{0}, \mathbf{c}_{1}\right) \in \operatorname{Spc}$ denote the mapping space between them.

For an object $\mathbf{c} \in \mathbf{C}$ we let $\mathbf{C}_{\mathbf{c}}$ ( (resp., $\mathbf{C}_{\text {/c }}$ ) denote the corresponding under-category (resp., overcategory).

In several places in the paper we will need the notion of left (resp., right) Kan extension. Let $F: \mathbf{C} \rightarrow \mathbf{D}$ be a functor, and let $\mathbf{E}$ is an $\infty$-category with colimits. Then the functor

$$
\operatorname{Funct}(\mathbf{D}, \mathbf{E}) \stackrel{\circ F}{\longrightarrow} \operatorname{Funct}(\mathbf{C}, \mathbf{E})
$$

admits a left adjoint, called the functor of left Kan extension along $F$.

For $\Phi \in \operatorname{Funct}(\mathbf{C}, \mathbf{E})$, the value of its left Kan extension on $\mathbf{d} \in \mathbf{D}$ is calculated by the formula

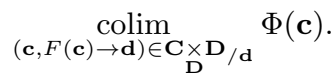

The notion of right Kan extension is obtained similarly: it is the right adjoint of (0.7); the formula for it is given by

$$
\lim _{(\mathbf{c}, \mathbf{d} \rightarrow F(\mathbf{c})) \in \mathbf{C}_{\mathbf{D}} \times \mathbf{D}_{\mathbf{d}} /} \Phi(\mathbf{d}) .
$$

We let DGCat denote the $\infty$-category of DG categories over $E$, see [GR2, Chapter 1, Sect. 10]. We assume all our DG categories to be cocomplete and we allow only colimit-preserving functors as 1-morphisms.

0.5.6. Prestacks. By definition, a prestack is a functor

$$
\left(\mathrm{Sch}^{\mathrm{aff}}\right)^{\mathrm{op}} \rightarrow \mathrm{Spc} \text {. }
$$

There will be two types of prestacks in this paper: the "source" type and the "target" type. The source type will be various geometric objects associated to the group $G$ and the curve $X$, such as the Ran space, affine Grassmannian $\operatorname{Gr}_{G}$, the loop group $\mathfrak{L}(G)$, etc. These prestacks have the feature that the corresponding functors on $\left(\mathrm{Sch}^{\text {aff }}\right)^{\text {op }}$ take values in the full subcategory

$$
\text { Sets } \subset \mathrm{Spc} .
$$

There will be a few other source prestacks (such as $\mathrm{Bun}_{G}$ or quotients of $\mathrm{Gr}_{G}$ by groups acting on it) and they will have the feature that the corresponding functors on $\left(\mathrm{Sch}^{\text {aff }}\right)^{\text {op }}$ take values in the full subcategory of Spc spanned by 1-groupoids (these are spaces $\mathcal{S}$, for which for any choice of $s: * \rightarrow \mathcal{S}$, the homoropy groups $\pi_{i}(S, s)$ vanish for $\left.i>1\right)$.

When we talk about the category of sheaves on a prestack, the prestack in question will be typically of the source type.

The target prestacks will be of the form $B^{n}(\mathcal{A})$ (see Sect. 1.3 .7 below), where $\mathcal{A}$ is a prestack that takes a constant value $A$, where $A$ is a discrete abelian group (or its sheafification in, say, the étale topology, denoted $B_{\text {et }}^{n}(\mathcal{A})$ ). Such prestacks take values in $n$-truncated spaces and they form a $(n+1)$ category. When $n$ is small, they can be described in a hands-on way by specifying objects, 1-morphisms, 2 -morphisms, etc; in this paper $n$ will be $\leq 4$, and in most cases $\leq 2$.

For example, we will often use the notion of a multiplicative $A$-gerbe on a group-prestack $\mathcal{H}$. Such an object is the same as a map of group-prestacks

$$
\mathcal{H} \rightarrow B_{\text {et }}^{2}(A) .
$$


0.6. Acknowledgements. The first author like to thank J. Lurie for many illuminating discussions related to factorization gerbes.

We would like to thank A. Beilinson, D. Clausen and M. Groechenig for helping us with K-theory.

We would like to thank the referee for some very helpful comments.

\section{Preliminaries}

This section is included for the reader's convenience: we review some constructions in algebraic geometry that involve higher category theory. The reader having a basic familiarity with this material should feel free to skip it.

1.1. The sheaf-theoretic context. Although most of this paper is devoted to the study of gerbes, we need to discuss categories of sheaves on various geometric objects because they appear in the formulation of metaplectic geometric Satake.

1.1.1. This note takes as an input a sheaf theory in the sense of [Ga2, Sect. 1.1] . A sheaf theory is a right-lax symmetric monoidal functor (see [GR2, Chapter 1, Sect. 3.2] for what this means)

$$
\text { Shv }:\left(\mathrm{Sch}_{\mathrm{ft}}^{\mathrm{aff}}\right)^{\mathrm{op}} \rightarrow \text { DGCat, }
$$

where DGCat is the $\infty$-category of (presentable) DG categories over a fixed field of coeffiecients $E$, assumed algebraically closed and of characteristic 0 . We stipulate that

$$
\operatorname{Shv}(\mathrm{pt})=\text { Vect }
$$

where Vect is the DG category of vector spaces over $E$. We also require that the functor (1.1) satifies étale descent.

Thus, to an affine scheme of finite type $S$ we assign a DG-category $\operatorname{Shv}(S)$, and to a morphism $f: S_{1} \rightarrow S_{2}$ a colimit-preserving pullback functor

$$
f^{!}: \operatorname{Shv}\left(S_{2}\right) \rightarrow \operatorname{Shv}\left(S_{1}\right)
$$

The right-lax symmetric monoidal structure on Shv amounts to a compatible collection of functors

$$
\otimes: \operatorname{Shv}\left(S_{1}\right) \otimes \operatorname{Shv}\left(S_{2}\right) \rightarrow \operatorname{Shv}\left(S_{1} \times S_{2}\right)
$$

1.1.2. The examples of sheaf theories that we are interested in are:

(a) For any ground field $k$ let $E=\overline{\mathbb{Q}}_{\ell}$, where $\ell$ is assumed to be invertible in $k$. First, for a finite extension $E^{\prime}$ of $\mathbb{Q}_{\ell}$ we consider the category $\operatorname{Shv}\left(S, E^{\prime}\right)$ equal to the ind-completion of the category of constructible étale sheaves on $S$ with $E^{\prime}$-coefficients, see [GL1, Sect. 2.3]. We take $\operatorname{Shv}(S)$ to be the colimit of these categories over $E^{\prime} \subset E$.

(b) When the ground field is $\mathbb{C}$, then for an arbitrary algebraically closed field $E$ of characteristic 0 , we can take $\operatorname{Shv}(S)$ to be the ind-completion of the category of constructible sheaves ${ }^{5}$ on $S$ with E-coefficients.

(c) When the ground field $k$ has characteristic 0 , and $E=k$, we take $\operatorname{Shv}(S)$ to be the category of D-modules on $S$.

\footnotetext{
${ }^{5}$ By a constructible sheaf on $S$ we mean a complex $\mathcal{F}$ of sheaves, for which there exists an algebraic stratification $S_{\alpha}$ of $S$, such that the restrictions $\left.\mathcal{F}\right|_{S_{\alpha}}$ are locally constant (in the analytic topology) with finite-dimensional fibers.
} 
1.1.3. The category of prestacks locally of finite type, denoted PreStk $\mathrm{l}_{\mathrm{lft}}$, is by definition that of all functors

$$
\left(\mathrm{Sch}_{\mathrm{ft}}^{\mathrm{aff}}\right)^{\mathrm{op}} \rightarrow \mathrm{Spc} .
$$

Yoneda embedding is a fully faithful functor

$$
\mathrm{Sch}_{\mathrm{ft}}^{\mathrm{aff}} \hookrightarrow \text { PreStk}_{\mathrm{lft}} .
$$

The right Kan extension of Shv along the (opposite of the) Yoneda embedding $\left(\mathrm{Sch}_{\mathrm{ft}}^{\text {aff }}\right)^{\mathrm{op}} \rightarrow$ $(\text { PreStk })^{\text {op }}$ defines a functor

$$
\text { Shv : }\left(\text { PreStk }_{l f t}\right)^{\text {op }} \rightarrow \text { DGCat . }
$$

Thus, if $y \in$ PreStk $_{\text {lft }}$ is written as

$$
y=\underset{i}{\operatorname{colim}} S_{i}, \quad S_{i} \in \operatorname{Sch}_{\mathrm{ft}}^{\mathrm{aff}}
$$

we have by definition

$$
\operatorname{Shv}(y)=\lim _{i} \operatorname{Shv}\left(S_{i}\right)
$$

1.2. What about non-finite type? At a certain point in this paper we will encounter the loop group $G((t))$, along with its various subgroups $N((t)), G \llbracket t \rrbracket$. In order to extend our sheaf theory to these objects, we proceed as follows.

1.2.1. We consider the category $\mathrm{Sch}^{\text {aff }}$ of all affine schemes. Note that $\mathrm{Sch}^{\text {aff }}$ identifies with $\operatorname{Pro}\left(\operatorname{Sch}_{\mathrm{ft}}^{\text {aff }}\right)$.

The operation of left Kan extension of (1.1) along $\left(\mathrm{Sch}_{\mathrm{ft}}^{\mathrm{aff}}\right)^{\mathrm{op}} \hookrightarrow\left(\mathrm{Sch}^{\mathrm{aff}}\right)^{\mathrm{op}}$ defines a functor

$$
\text { Shv }:\left(\mathrm{Sch}^{\text {aff }}\right)^{\text {op }} \rightarrow \text { DGCat . }
$$

Thus, if an affine scheme $S$ is written as a (filtered) limit

$$
S=\lim _{\alpha} S_{\alpha}, \quad S_{\alpha} \in \operatorname{Sch}_{\mathrm{ft}}^{\mathrm{aff}}
$$

we have by definition

$$
\operatorname{Shv}(S)=\underset{\alpha}{\operatorname{colim}} \operatorname{Shv}\left(S_{\alpha}\right) .
$$

The functor (1.2) inherits a right-lax symmetric monoidal structure.

1.2.2. Let PreStk denote the category of all $\left(\right.$ accessible $\left.^{6}\right)$ functors

$$
\left(\mathrm{Sch}^{\text {aff }}\right)^{\text {op }} \rightarrow \mathrm{Spc} \text {. }
$$

We define the functor

$$
\text { Shv : }\left(\text { PreStk) }{ }^{\text {op }} \rightarrow\right. \text { DGCat }
$$

to be the right Kan extension of (1.2) along the Yoneda embedding Sch $^{\text {aff }} \hookrightarrow$ PreStk.

1.3. Digression: some higher algebra. To facilitate the reader's task, in this subsection we will review some notions from higher algebra that will be used in this paper. The main reference for this material is [Lu2].

1.3.1. Monoids and groups. In any $\infty$-category $\mathbf{C}$ that contains finite products (including the empty finite product, i.e., a final object), it makes sense to consider the category $\operatorname{Monoid}(\mathbf{C})$ of monoid-objects in $\mathbf{C}$. This is a full subcategory in the category of simplicial objects of $\mathbf{C}$ (i.e., Funct $\left.\left(\boldsymbol{\Delta}^{\mathrm{op}}, \mathbf{C}\right)\right)$ that consists of objects, satisfying the Segal condition. Similarly, one defines the category commutative monoids ComMonoid(C) in $\mathbf{C}$.

For example, take $\mathbf{C}=\infty$-Cat. In this way we obtain the notion of monoidal (resp., symmetric monoidal) category.

\footnotetext{
${ }^{6}$ Ignore this adjective if you do not want to worry about set theory.
} 
1.3.2. If $\mathbf{c}$ is an object of an $\infty$-category $\mathbf{C}$, then $\operatorname{Maps}_{\mathbf{C}}(\mathbf{c}, \mathbf{c})$ has a natural structure of monoid in Spc.

For $H \in \operatorname{Monoid}(\mathrm{Spc})$, an action of $H$ on $\mathbf{c}$ is by definition a homomorphism $H \rightarrow \operatorname{Maps}_{\mathbf{C}}(\mathbf{c}, \mathbf{c})$ of monoid objects in Spc.

1.3.3. The $\infty$-category Monoid(C) (resp., ComMonoid $(\mathbf{C})$ ) contains the full subcategory of group-like objects, denoted $\operatorname{Grp}(\mathbf{C})$ (resp., $\operatorname{ComGrp}(\mathbf{C}))$.

Let $\operatorname{Ptd}(\mathbf{C})$ be the category of pointed objects in $\mathbf{C}$, i.e., $\mathbf{C}_{* /}$, where $*$ denotes the final object in C. We have the loop functor

$$
\Omega: \operatorname{Ptd}(\mathbf{C}) \rightarrow \operatorname{Grp}(\mathbf{C}), \quad(* \rightarrow \mathbf{c}) \mapsto \underset{\mathbf{c}}{\times} *
$$

The left adjoint of this functor (if it exists) is called the functor of the classifying space and is denoted

$$
H \mapsto B(H)
$$

1.3.4. For $\mathbf{C}=\operatorname{Spc}$ (or $\mathbf{C}=\operatorname{Funct}(\mathbf{D}, \mathrm{Spc}$ ) for some other category $\mathbf{D}$, or any topos), the functor $B$ does exist and is fully faithful. The essential image of $B: \operatorname{Grp}(\mathrm{Spc}) \rightarrow \operatorname{Ptd}(\mathrm{Spc})$ consists of connected spaces.

For an object $\mathcal{S} \in \operatorname{Ptd}(\mathrm{Spc})$, its $i$-th homotopy group $\pi_{i}(\mathcal{S})$ is defined to be

$$
\pi_{0}\left(\Omega^{i}(\mathcal{S})\right)
$$

where $\Omega^{i}(\mathcal{S})$ is viewed as a mere object of Spc.

1.3.5. For $\mathbf{C}$ as above, an object $\mathbf{c} \in \mathbf{C}$ and $H \in \operatorname{Grp}(\mathbf{C})$, one defines the notion of action of $H$ on $\mathbf{C}$. By definition, such a data consists of an object $\widetilde{\mathbf{c}} \in \mathbf{C}_{/ B(H)}$ together with an identification

$$
\mathbf{c} \simeq \underset{B(H)}{\times} \widetilde{\mathbf{c}} .
$$

For $\mathbf{C}=$ Spc, this coincides with the notion from Sect. 1.3.2.

1.3.6. For $k \geq 0$, we introduce the category $\mathbb{E}_{k}(\mathbf{C})$ of $\mathbb{E}_{k}$-objects in $\mathbf{C}$ inductively, by setting

$$
\mathbb{E}_{0}(\mathbf{C})=\operatorname{Ptd}(\mathbf{C})
$$

and

$$
\mathbb{E}_{k}(\mathbf{C})=\operatorname{Monoid}\left(\mathbb{E}_{k-1}(\mathbf{C})\right) .
$$

Let $\mathbb{E}_{k}^{\text {grp-like }}(\mathbf{C}) \subset \mathbb{E}_{k}(\mathbf{C})$ the full subcategory of group-like objects, defined to be the preimage of

$$
\operatorname{Grp}(\mathbf{C}) \subset \operatorname{Monoid}(\mathbf{C})=\mathbb{E}_{1}(\mathbf{C})
$$

under any of the $k$ possible forgetful functors $\mathbb{E}_{k}(\mathbf{C}) \rightarrow \mathbb{E}_{1}(\mathbf{C})$.

We have a pair of mutually adjoint functors

$$
B: \mathbb{E}_{k}^{\text {grp-like }}(\mathbf{C}) \rightleftarrows \mathbb{E}_{k-1}^{\text {grp-like }}(\mathbf{C}): \Omega
$$

for any $k \geq 2$.

For $i \leq k$ we let $B^{i}$ denote the resulting functor

$$
\mathbb{E}_{k}^{\text {grp-like }}(\mathbf{C}) \rightarrow \mathbb{E}_{k-i}^{\text {grp-like }}(\mathbf{C}) .
$$


1.3.7. One shows that the forgetful functor

$$
\operatorname{Monoid}(\operatorname{ComMonoid}(\mathbf{C})) \rightarrow \operatorname{ComMonoid}(\mathbf{C})
$$

is an equivalence.

This implies that for every $k$ we have a canonically defined functor

$$
\operatorname{ComMonoid}(\mathbf{C}) \rightarrow \mathbb{E}_{k}(\mathbf{C}),
$$

and these functors are compatible with the forgetful functors $\mathbb{E}_{k}(\mathbf{C}) \rightarrow \mathbb{E}_{k-1}(\mathbf{C})$. Thus, we obtain a canonically defined functor

$$
\operatorname{ComMonoid}(\mathbf{C}) \rightarrow \mathbb{E}_{\infty}(\mathbf{C}):=\lim _{\longleftarrow} \mathbb{E}_{k}(\mathbf{C})
$$

It is known (see [Lu2, Remark 5.2.6.26]) that the functor (1.3) is an equivalence.

The category

$$
\operatorname{ComGrp}(\mathrm{Spc}) \simeq \mathbb{E}_{\infty}^{\text {grp-like }}(\mathrm{Spc})
$$

identifies with that of connective spectra.

For any $i \geq 0$, we have the mutually adjoint endo-functors

$$
B^{i}: \operatorname{ComGrp}(\mathrm{Spc}) \rightleftarrows \operatorname{ComGrp}(\mathrm{Spc}): \Omega^{i}
$$

with $B^{i}$ being fully faithful.

1.3.8. Let $A$ be an object of $\mathbb{E}_{2}^{\text {grp-like }}(\mathrm{Spc})$, so that $B(A)$ is an object of $\operatorname{Grp}(\mathrm{Spc})$.

By an action of $A$ on an $\infty$-category $\mathbf{C}$ we shall mean an action of $B(A)$ on $\mathbf{C}$ as an object of $\infty$-Cat.

For example, taking $A=E^{\times} \in \operatorname{ComGrp}(\mathrm{Spc})$, we obtain an action of $E^{\times}$on any DG category. Explicitly, we identify $B\left(E^{\times}\right)$with the space of $E^{\times}$-torsors, i.e., lines, and the action in question sends a line $\ell$ to the endofunctor

$$
\mathbf{c} \mapsto \ell \otimes \mathbf{c}
$$

\subsection{Gerbes.}

1.4.1. Let $y$ be a prestack, and let $\mathcal{A}$ be a group-like $\mathbb{E}_{n}$-object in the category PreStk/y, for $n \geq 1$. In other words, for a given $(S \stackrel{y}{\rightarrow} y) \in\left(\mathrm{Sch}^{\text {aff }}\right) / y$, the space

$$
\operatorname{Maps}(S, \mathcal{A}) \underset{\operatorname{Maps}(S, y)}{\times}\{y\}
$$

is a group-like $\mathbb{E}_{n}$-object of $\mathrm{Spc}$, in a way functorial in $(S, y)$.

We include the case of $n=\infty$, when we stipulate that $\mathcal{A}$ is a commutative group-object of PreStk/y. I.e., (1.4) should be a commutative group-object of Spc, i.e., a connective spectrum.

For any $0 \leq i \leq n$, we let $B^{i}(\mathcal{A})$ denote the $i$-fold classifying space of $\mathcal{A}$. This is a group-like

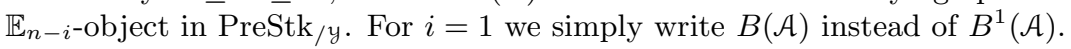

1.4.2. We let $B_{\text {et }, y}^{i}(\mathcal{A})$ (resp., $B_{\text {Zar, } / y}^{i}(\mathcal{A})$ ) denote the étale (resp., Zariski) sheafification of $B^{i}(\mathcal{A})$ in the category $\left(\mathrm{Sch}^{\text {aff }}\right) / y$ (see [GR2, Chapter 2, Sect. 2.3]). We will be interested in spaces of the form

$$
\operatorname{Maps}_{/ y}\left(y, B_{\mathrm{et}, / y}^{i}(\mathcal{A})\right),
$$

where $\operatorname{Maps}_{/ y}(-,-)$ is short-hand for $\operatorname{Maps}_{\operatorname{PreStk}_{/ y}}(-,-)$.

Note that (1.5) is naturally a group-like $\mathbb{E}_{n-i}$-space (resp., a commutative group object in Spc if $n=\infty)$. 
1.4.3. In most examples, we will take $\mathcal{A}$ to be of the form $A \times y$, where $A$ is a torsion abelian group, considered as a constant prestack. In this case

$$
\operatorname{Maps}_{/ y}\left(y, B_{\mathrm{et}, / y}^{i}(\mathcal{A})\right) \simeq \operatorname{Maps}\left(y, B_{\mathrm{et}}^{i}(A)\right) .
$$

Note that

$$
\pi_{j}\left(\operatorname{Maps}\left(y, B_{\mathrm{et}}^{i}(A)\right)\right)=\left\{\begin{array}{l}
H_{\mathrm{et}}^{i-j}(y, A), \quad j \leq i \\
0, \quad j>i .
\end{array}\right.
$$

Here $H_{\mathrm{et}}^{\bullet}(y, A)$ refers to the étale cohomology of $y$ with coefficients in $A$. In other words, it is the cohomology of the object

see [GL2, Sect. 2.3].

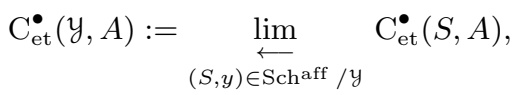

Note also that in this case the functor

$$
S \mapsto \operatorname{Maps}\left(S, B_{\text {et }}^{i}(A)\right), \quad\left(\operatorname{Sch}^{\text {aff }}\right)^{\text {op }} \rightarrow \operatorname{Spc}
$$

identifies with the left Kan extension of its restriction to $\left(\operatorname{Sch}_{\mathrm{ft}}^{\text {aff }}\right)^{\text {op }}$. I.e., if an affine scheme $S$ is written as a (filtered) limit

$$
S=\lim _{\alpha} S_{\alpha}, \quad S_{\alpha} \in \mathrm{Sch}_{\mathrm{ft}}^{\mathrm{aff}}
$$

then the map

$$
\underset{\alpha}{\operatorname{colim}} \operatorname{Maps}\left(S_{\alpha}, B_{\text {et }}^{i}(A)\right) \rightarrow \operatorname{Maps}\left(S, B_{\text {et }}^{i}(A)\right)
$$

is an isomorphism ${ }^{7}$.

1.4.4. For $k=1$, the points of the space

$$
\operatorname{Tors}_{\mathcal{A}}(y):=\operatorname{Maps}_{/ y}\left(y, B_{\text {et }, / y}(\mathcal{A})\right)
$$

are by definition $\mathcal{A}$-torsors on $y$.

1.4.5. Our primary interest is the cases of $k=2$. We will call objects of the space

$$
\operatorname{Ge}_{\mathcal{A}}(y):=\operatorname{Maps}_{/ y}\left(y, B_{\text {et }, / y}^{2}(\mathcal{A})\right) \text {. }
$$

$\mathcal{A}$-gerbes on $y$.

When $\mathcal{A}$ is of the form $A \times y$ (see Sect. 1.4.3 above), we will simply write $\operatorname{Ge}_{A}(y)$.

1.5. Gerbes coming from line bundles. In this subsection we will be studying gerbes for a constant commutative group-prestack, corresponding to a torsion abelian group $A$.

1.5.1. Let $A(-1)$ denote the group

$$
\underset{n}{\operatorname{colim}} \operatorname{Hom}\left(\mu_{n}, A\right)
$$

Here $\mu_{n}$ is the group of $n$-th roots of unity in $k$, where the integer $n$ is assumed invertible in $k$. The above colimit is taken with respect to the maps

$$
\mu_{n^{\prime}} \stackrel{x \mapsto x^{\frac{n^{\prime}}{n}}}{\rightarrow} \mu_{n}, \quad \text { for } n \mid n^{\prime} .
$$

For future reference, denote also

$$
A(1)=\underset{n}{\operatorname{colim}} \mu_{n} \underset{\mathbb{Z}}{\otimes} A,
$$

where colimit is taken with respect to the maps

$$
\mu_{n} \hookrightarrow \mu_{n^{\prime}}, \quad \text { for } n \mid n^{\prime} .
$$

\footnotetext{
${ }^{7}$ The latter assertion means that $B_{\mathrm{et}}^{i}(A)$ is locally of finite type as a prestack.
} 
1.5.2. We claim that to any line bundle $\mathcal{L}$ on a prestack $y$ and an element $a \in A(-1)$ one can canonically associate an $A$-gerbe, denoted $\mathcal{L}^{a}$, over $y$.

It suffices to perform this construction for $A=\mu_{n}$ and $a$ coming from the identity map $\mu_{n} \rightarrow \mu_{n}$. In this case, the corresponding $\mu_{n}$-gerbe will be denoted $\mathcal{L}^{\frac{1}{n}}$.

By definition, for an affine test scheme $S$ over $y$, the value of $\mathcal{L}^{\frac{1}{n}}$ on $S$ is the groupoid of pairs

$$
\left(\mathcal{L}^{\prime},\left.\left(\mathcal{L}^{\prime}\right)^{\otimes n} \simeq \mathcal{L}\right|_{S}\right),
$$

where $\mathcal{L}^{\prime}$ is a line bundle on $S$.

Note that if $\mathcal{L}$ admits an $n$-th root $\mathcal{L}^{\prime}$, then this $\mathcal{L}^{\prime}$ determines a trivialization of $\mathcal{L}^{\frac{1}{n}}$.

Remark 1.5.3. We emphasize the notational difference between the $\mu_{n}$-gerbe $\mathcal{L}^{\frac{1}{n}}$, and the line bundle

$\mathcal{L}^{\otimes \frac{1}{n}}$, when the latter happens to exist. Namely, a choice of $\mathcal{L}^{\otimes \frac{1}{n}}$ defines a trivialization of the gerbe $\mathcal{L}^{\frac{1}{n}}$.

1.5.4. Let $Y$ be a smooth scheme, and let $Z \subset Y$ be a subvariety of codimension one. Let $Z_{i}, i \in I$ denote the irreducible components of $Z$. For every $i$, let $\mathcal{O}\left(Z_{i}\right)$ denote the corresponding line bundle on $Y$, trivialized away from $Z$.

We obtain a homomorphism

$$
\operatorname{Maps}(I, A(-1)) \rightarrow \operatorname{Ge}_{A}(Y) \underset{\operatorname{Ge}_{A}(Y-Z)}{\times} *, \quad\left(I \mapsto a_{i}\right) \rightsquigarrow \bigotimes_{i} \mathcal{O}\left(Z_{i}\right)^{a_{i}}
$$

Lemma 1.5.5. Assuming that the orders of elements in $A$ are prime to char $(k)$, i.e., that A has no $p$-torsion, where $p=\operatorname{char}(k)$. Then the map (1.8) is an isomorphism in Spc.

Proof. The assertion follows from the fact that the étale cohomology group $H_{\mathrm{et}, Z}^{i}(Y, A)$ identifies with $\operatorname{Maps}(I, A(-1))$ for $i=2$ and vanishes for $i=1,0$.

1.6. Presheaves of categories. (Pre)sheaves of categories appear in this paper as a language in which we formulate the metaplectic geometric Satake functor. The reader can skip this subsection on the first pass, and return to it when necessary.

The discussion in this section is essentially borrowed from [Ga1, Sect. 1.1].

1.6.1. Note that the diagonal morphism for affine schemes defines on every object of $\left(\operatorname{Sch}^{\text {aff }}\right)^{\text {op }}$ a canonical structure of commutative algebra.

Hence, the right-lax symmetric monoidal structure on Shv naturally gives rise to a functor

$$
\left(\mathrm{Sch}^{\text {aff }}\right)^{\text {op }} \rightarrow \text { ComAlg }(\text { DGCat })=: \text { DGCat }{ }^{\text {SymMon }} .
$$

In particular, for every $S \in \mathrm{Sch}^{\text {aff }}$, the category $\operatorname{Shv}(S)$ has a natural symmetric monoidal structure, and for every $f: S_{1} \rightarrow S_{2}$, the functor $f^{!}: \operatorname{Shv}\left(S_{2}\right) \rightarrow \operatorname{Shv}\left(S_{1}\right)$ is symmetric monoidal.

1.6.2. By a presheaf of DG categories $\mathcal{C}$ over $y \in$ PreStk we will mean a functorial assignment

$$
(S \stackrel{y}{\rightarrow} y) \in\left(\left(\operatorname{Sch}^{\text {aff }}\right) / y\right)^{\text {op }} \rightsquigarrow \mathcal{C}(S, y) \in \operatorname{Shv}(S) \text {-mod, }
$$

where $\operatorname{Shv}(S)$-mod denotes the category of modules in the (symmetric) monoidal category DGCat for the (commutative) algebra object $\operatorname{Shv}(S)$.

A basic example of a sheaf of categories is Shv/y, defined by setting

$$
\operatorname{Shv}_{/ y}(S, y):=\operatorname{Shv}(S) \text {. }
$$

1.6.3. An example. Let $Z$ be a prestack over $y$. We define a presheaf of categories $\operatorname{Shv}(Z) / y$ over $y$ by setting for $S \stackrel{y}{\rightarrow} y$,

$$
\operatorname{Shv}(Z) / y(S, y)=\operatorname{Shv}(S \underset{y}{\times} Z)
$$


1.6.4. Part of the data of a presheaf of DG categories is a compatibility of actions for morphisms between affine schemes:

For $f: S_{1} \rightarrow S_{2}, y_{2}: S_{2} \rightarrow y$ and $y_{1}=y_{2} \circ f$, the corresponding functor

$$
\mathcal{C}\left(S_{2}, y_{2}\right) \rightarrow \mathcal{C}\left(S_{1}, y_{1}\right)
$$

must intertwine the action of $\operatorname{Shv}\left(S_{2}\right)$ on $\mathcal{C}\left(S_{2}, y_{2}\right)$ with the action of $\operatorname{Shv}\left(S_{1}\right)$ on $\mathcal{C}\left(S_{1}, y_{1}\right)$ via the monoidal functor $f^{!}: \operatorname{Shv}\left(S_{2}\right) \rightarrow \operatorname{Shv}\left(S_{1}\right)$.

In particular, the functor (1.9) gives rise to a functor of $\operatorname{Shv}\left(S_{1}\right)$-module categories

$$
\operatorname{Shv}\left(S_{1}\right) \underset{\operatorname{Shv}\left(S_{2}\right)}{\otimes} \mathcal{C}\left(S_{2}, y_{2}\right) \rightarrow \mathcal{C}\left(S_{1}, y_{1}\right),
$$

where $\otimes$ is the operation of tensor product of DG categories (see, e.g., [GR2, Chapter 1, Sect. 10.4]).

1.6.5. We will say that a presheaf of DG categories is quasi-coherent if the functors (1.10) are equivalences for all $f: S_{1} \rightarrow S_{2}$.

Typically, presheaves of categories of the form of Sect. 1.6.3 are not quasi-coherent, even if the morphism $Z \rightarrow y$ is schematic. This is because in the context of $\ell$-adic sheaves, for a pair of affine schemes $S_{1}$ and $S_{2}$, the functor

$$
\operatorname{Shv}\left(S_{1}\right) \otimes \operatorname{Shv}\left(S_{2}\right) \rightarrow \operatorname{Shv}\left(S_{1} \times S_{2}\right)
$$

is fully faithful, but not an equivalence (however, it is an equivalence in the context of D-modules).

1.6.6. Forgetting the module structure, a presheaf of DG categories $\mathcal{C}$ over $y$ defines a functor

$$
\left(\left(\mathrm{Sch}^{\mathrm{aff}}\right) / y\right)^{\mathrm{op}} \rightarrow \text { DGCat } .
$$

We shall say that $\mathcal{C}$ is a sheaf if it satisfies étale descent, i.e., if the functor (1.11) satisfies étale descent.

For example, presheaves of categories arising as in Sect. 1.6.3 are sheaves of categories.

1.6.7. Applying to the functor (1.11) the procedure of right Kan extension along

$$
\left(\left(\mathrm{Sch}^{\mathrm{aff}}\right) / y\right)^{\mathrm{op}} \rightarrow((\text { PreStk }) / y)^{\mathrm{op}}
$$

we obtain that for every prestack $Z$ over $y$ there is a well-defined DG category $\mathrm{C}(Z)$.

Namely, if

$$
Z \simeq \underset{i}{\operatorname{colim}} S_{i}, \quad\left(S_{i}, y_{i}\right) \in\left(\operatorname{Sch}^{\mathrm{aff}}\right) / y
$$

then

$$
\mathrm{e}(Z)={\underset{\leftarrow}{\leftarrow}}_{\lim } \mathrm{e}\left(S_{i}, y_{i}\right)
$$

We will refer to $\mathcal{C}(Z)$ as the "category of sections of $\mathcal{C}$ over $Z$ ". By construction the DG category $\mathcal{C}(Z)$ is naturally an object of $\operatorname{Shv}(Z)$-mod.

1.6.8. When $Z$ is $y$ itself, we will refer to $\mathcal{C}(y)$ as the "category of global sections of $\mathcal{C}$ ".

For $\mathcal{C}$ as in Sect. 1.6.3, we have

$$
\mathcal{C}(y) \simeq \operatorname{Shv}(Z)
$$

1.7. Some twisting constructions. The material in this subsection may not have proper references in the literature, so we provide some details. The reader is advised to skip it and return to it when necessary. 
1.7.1. Twisting by a torsor. Let $y$ be a prestack, and let $\mathcal{H}$ (resp., $\mathcal{F}$ ) a group-like object in PreStk/y (resp., an object in PreStk/y, equipped with an action of $\mathcal{H}$ ). In other words, these are functorial assignments

$$
(S, y) \in\left(\mathrm{Sch}^{\mathrm{aff}}\right) / y \rightsquigarrow \mathcal{H}(S, y) \in \mathrm{Grp}(\mathrm{Spc}), \quad(S, y) \in\left(\mathrm{Sch}^{\mathrm{aff}}\right) / y \rightsquigarrow \mathcal{F}(S, y) \in \mathrm{Spc}
$$

and an action of $\mathcal{H}(S, y)$ on $\mathcal{F}(S, y)$.

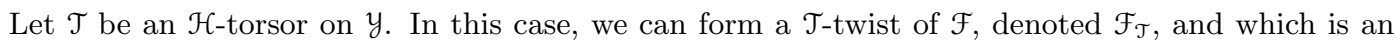
étale sheaf. Here is the construction ${ }^{8}$ :

Consider the category $\operatorname{Split}(\mathcal{T})$ formed by $(S, y) \in\left(\operatorname{Sch}^{\text {aff }}\right) / y$ equipped with a datum of a lift of the map $S \stackrel{y}{\rightarrow} y \stackrel{\mathcal{T}}{\rightarrow} B_{\text {et }}(\mathcal{H})$ to a map $z: S \rightarrow B(\mathcal{H})$. The forgetful functor $\operatorname{Split}(\mathcal{T}) \rightarrow\left(\operatorname{Sch}^{\text {aff }}\right) / y$ forms a basis of the étale topology, so it is sufficient to specify the restriction of $\mathcal{F}_{\mathcal{T}}$ to $\operatorname{Split}(\mathcal{T})$.

We interpret the data of the action of $\mathcal{H}$ on $\mathcal{F}$ as an object $\widetilde{\mathcal{F}} \in \operatorname{PreStk}_{/ B(\mathcal{H})}$ (see Sect. 1.3.5). The sought-for functor $\left.\mathcal{F}_{\mathcal{T}}\right|_{\text {Split }(\mathcal{T})}$ is given by sending $(S, y, z)$ to

$$
* \underset{B(\mathcal{H})(S, y)}{\times} \tilde{\mathcal{F}}(S, y)
$$

where $* \in B(\mathcal{H})(S, y)$ corresponds to the given map $z$.

1.7.2. A twist of a presheaf of categories by a gerbe. Let now $\mathcal{C}$ be a presheaf of DG categories over $y$, and let $\mathcal{A}$ be a group-like $\mathbb{E}_{2}$-object in PreStk/y.

Let us be given an action of $\mathcal{A}$ on $\mathcal{C}$. In other words, we are given a functorial assignment for every $(S, y) \in\left(\mathrm{Sch}^{\text {aff }}\right) / y$ of an action of $\mathcal{A}(S, y)$ on $\mathcal{C}(S, y)$, see Sect. 1.3.8. In particular, we obtain that the prestack of groups $B(\mathcal{A})$ acts on $\mathcal{C}$.

Let $\mathcal{G}$ be a $\mathcal{A}$-gerbe on $y$, i.e., a $B(\mathcal{A})$-torsor. Repeating the construction of Sect. 1.7.1, we obtain that we can form the twist $\mathcal{C}_{\mathcal{G}}$ of $\mathcal{C}$ by $\mathcal{G}$, which is a sheaf of DG categories over $y$.

Explicitly, for every $(S \stackrel{y}{\rightarrow} y) \in\left(\operatorname{Sch}^{\text {aff }}\right) / y$ and a trivialization of $\left.\mathcal{G}\right|_{S}$ we have an identification

$$
\mathrm{C}_{\mathcal{G}}(S, y) \simeq \mathcal{C}(S, y)
$$

The effect of change of trivialization by a point $a \in \mathcal{A}(S, y)$ has the effect of action of

$$
a \in \operatorname{End}\left(\operatorname{Id}_{\mathcal{C}}(S, y)\right) \text {. }
$$

1.7.3. Let $E^{\times, \text {tors }}$ denote the subgroup of elements of $E^{\times}$that have a finite order prime to char $(k)$.

Let us take $\mathcal{A}$ to be the constant group-prestack $y \times E^{\times, \text {tors }}$. In this case, the tautological embedding $E^{\times, \text {tors }} \rightarrow E^{\times}$gives rise to an action of $\mathcal{A}$ on any presheaf of DG categories.

Thus, for every $\mathcal{G} \in \mathrm{Ge}_{E^{\times} \text {,tors }}(y)$ and any presheaf of categories $\mathcal{C}$ over $y$, we can form its twisted version $\mathcal{C}_{\mathcal{G}}$

1.7.4. The category of sheaves twisted by a gerbe. We apply the above construction to $\mathcal{C}:=\mathrm{Shv} / \mathrm{y}$. Thus, for any $(S, y) \in\left(\operatorname{Sch}^{\text {aff }}\right) / y$ we have the twisted version of the category $\operatorname{Shv}(S), \operatorname{denoted} \operatorname{Shv}_{\mathcal{G}}(S)$.

As in Sect. 1.6.7, the procedure of right Kan extension defines the category

$$
\operatorname{Shv}_{\mathcal{G}}(Z)
$$

for any $Z \in \operatorname{PreStk} / y$.

\subsection{Character sheaves and twisted equivariance.}

${ }^{8}$ Note that when $\mathcal{T}$ is the trivial torsor, the output of this construction is the étale sheafification of $\mathcal{F}$. 
1.8.1. Recall that for $S \in \mathrm{Sch}^{\text {aff }}$, the category $\operatorname{Shv}(S)$ has a natural symmetric monoidal structure.

By a graded local system on $S$ we will mean an object of $\operatorname{Shv}(S)$ that is invertible in the sense of the above symmetric monoidal structure.

By a local system on $S$ we will mean a graded local system all of whose fibers are lines in cohomological degree 0.

(Graded) local systems on $S$ form a Picard category, i.e., a symmetric monoidal category in which every object is invertible.

1.8.2. Let $\mathcal{L S}$ denote the group object of PreStk that assigns to $S \in \mathrm{Sch}^{\text {aff }}$ the Picard category of 1-dimensional local systems (within $\operatorname{Shv}(S)$ ).

Let $H$ be another group-object of PreStk. By a character sheaf on $H$ we will mean a map of group prestacks $H \rightarrow \mathcal{L} \mathcal{S}$.

Let $y$ be a prestack acted on by $H$, which we interpret as a prestack $\widetilde{y}$, equipped with a map to $B(H)$. Given a chracter sheaf $\chi$ on $H$, we can thus view $\widetilde{y}$ as equipped with a map to $B(\mathcal{L} \mathcal{S})$, i.e., with an $\mathcal{L} \mathcal{S}$-torsor, denoted $\mathcal{T}_{\chi}$.

Note that the presheaf of categories $\mathrm{Shv}_{\tilde{y}}$ is naturally acted on by $\mathcal{L} \mathcal{S}$. Applying a variant of the twisting construction of Sect. 1.7.1, we obtain a twist of this sheaf of categories by the above $\mathcal{L} \mathcal{S}$-torsor $\mathcal{T}_{\chi}$.

In particular, for $(S, y) \in\left(\operatorname{Sch}^{\text {aff }}\right)_{/ \widetilde{y}}$, we obtain a well-defined category $\operatorname{Shv}_{\mathcal{T}_{\chi}}(S)$. By applying the procedure of right Kan extension, we obtain a well-defined category $\operatorname{Shv}_{\mathcal{T}_{\chi}}(Z)$ for any $Z \in \operatorname{PreStk}_{/ \widetilde{y}}$, and in particular for $Z=\widetilde{y}$.

We define the category of $(H, \chi)$-twisted equivariant sheaves on $y$ as

$$
\operatorname{Shv}(y)^{H, \chi}:=\operatorname{Shv}_{\mathcal{T}_{\chi}}(\widetilde{y})
$$

1.8.3. The above construction and one in Sect. 1.7.2 are interrelated. Namely, note that we have a tautological map of group prestacks

$$
B\left(E^{\times, \text {tors }}\right) \rightarrow \mathcal{L S}
$$

which extends to a map

$$
B_{\text {et }}\left(E^{\times, \text {tors }}\right) \rightarrow \mathcal{L} \mathcal{S}
$$

since $\mathcal{L} \mathcal{S}$ satisfies étale descent.

In particular, there exists a tautological character sheaf $\chi_{\text {taut }}$ over $B\left(E^{\times, \text {tors }}\right)$.

Given a prestack $y^{\prime}$ and a $E^{\times, \text {tors }}$-gerbe $\mathcal{G}$ over $y^{\prime}$, we can form the prestack

$$
y:=\operatorname{pt} \underset{B_{\text {et }}^{2}\left(E^{\times, \text {tors }}\right)}{\times} y^{\prime},
$$

equipped with an action of $B_{\text {et }}\left(E^{\times, \text {tors }}\right)$; this is the "total space" of $\mathcal{G}$;

We have

$$
\operatorname{Shv}_{\mathcal{G}}\left(y^{\prime}\right) \simeq \operatorname{Shv}(y)^{B_{\text {et }}\left(E^{\times, \text {tors }}\right), \chi_{\text {taut }}}
$$


1.8.4. An example. Let $n$ be an integer invertible in $k$ and let $a$ be an element of order $n$ in $\left(E^{\times, \text {tors }}\right)(-1)$, see Sect. 1.5.1.

The Kummer cover

$$
\mathbb{G}_{m} \stackrel{x \mapsto x^{n}}{\longrightarrow} \mathbb{G}_{m}
$$

defines a group homomorphism

$$
\mathbb{G}_{m} \rightarrow B_{\text {et }}\left(\mu_{n}\right) \text {. }
$$

Using the element $a$ we obtain a homomorphism

$$
\mathbb{G}_{m} \rightarrow B_{\text {et }}\left(E^{\times, \text {tors }}\right) .
$$

Let $\chi_{a}:=\left.\chi_{\text {taut }}\right|_{\mathbb{G}_{m}}$ denote the corresponding character sheaf (known as the Kummer sheaf) on $\mathbb{G}_{m}$.

Let $\mathcal{L}$ be a line bundle over a prestack $y$. Let $\mathcal{L}^{a}$ be the corresponding $E^{\times, \text {tors }}$-gerbe over $y$. Then the category $\operatorname{Shv}_{\mathcal{L}^{a}}(y)$ can be explicitly described as follows:

$$
\operatorname{Shv}_{\mathcal{L}^{a}}(y) \simeq \operatorname{Shv}(\mathcal{L}-\{0\})^{\mathbb{G}_{m}, \chi_{a}},
$$

where $\mathcal{L}-\{0\}$ is the total space of $\mathcal{L}$ with zero-section removed, viewed as a $\mathbb{G}_{m}$-torsor over $y$.

\subsection{Other sheaf-theoretic contexts.}

1.9.1. Suppose for a moment that our ground field $k$ is $\mathbb{C}$, and our sheaf theory is that of constructible sheaves with $E$-coefficients. When working with schemes of finite type, instead of considering the group $E^{\times, \text {tors }}$ and gerbes locally trivial in the étale topology, one can consider $E^{\times}$-gerbes locally trivial in the analytic topology.

For a prestack $y$, we denote the corresponding 2-groupoid of $E^{\times}$-gerbes by $\operatorname{Ge}_{E^{\times}}(y)$.

Given a prestack $y$ and $\mathcal{G} \in \operatorname{Ge}_{E^{\times}}(y)$, we have a well-defined functor

$$
\operatorname{Shv}_{\mathcal{G}}:\left(\operatorname{PreStk}_{/ y}\right)^{\mathrm{op}} \rightarrow \infty \text {-Cat. }
$$

If $\mathcal{L}$ is a line bundle on a prestack $y$ and $a$ is an element of $E^{\times}$, we let $\mathcal{L}^{a}$ denote the corresponding $E^{\times}$-gerbe on $y$.

The assertion of Lemma 1.5.5 holds mutatis mutandis. The rest of the theory is unchanged.

1.9.2. For a finite type scheme $S$ we have a canonical map

$$
\mathrm{Ge}_{E \times, \text { tors }}(S) \rightarrow \mathrm{Ge}_{E \times}(S) .
$$

At the level of $\pi_{0}$, the image of this map consists of torsion elements in $H_{\mathrm{an}}^{2}\left(S, E^{\times}\right)$.

Note, however, that the map (1.12) is not fully faithful: at the level of $\pi_{1}$ it corresponds to the map

$$
H_{\mathrm{et}}^{1}\left(S, E^{\times, \text {tors }}\right) \hookrightarrow H_{\mathrm{an}}^{1}\left(S, E^{\times}\right),
$$

whose image consists of torsion elements. In other words, automorphisms of a given $E^{\times}$-gerbe is the Picard category of all $E^{\times}$-torsors (i.e., 1-dimensional local systems with coefficients in $E$ ), and for a $E^{\times, \text {tors }}$-gerbe we allow those local systems that become trivial when raised to some power.

Note that in this case we can identify $E^{\times, \text {tors }}(-1)$ with $E^{\times, \text {tors }}$ itself; this is because the fundamental group of $\mathbb{G}_{m}$ is identified with $\mathbb{Z}$ via the exponential map. 
1.9.3. Let now $k$ be an arbitrary field of characteristic 0, and let our sheaf theory be that of Dmodules, so that $E=k$. Recall that for a scheme $S$ of finite type, the category $\operatorname{Shv}(S)=\mathrm{D}-\bmod (S)$ is by definition

$$
\mathrm{QCoh}\left(S_{\mathrm{dR}}\right)
$$

where $S_{\mathrm{dR}}$ is the de Rham prestack of $S$.

In this case, the counterpart of the notion of $E^{\times}$-gerbe from Sect. 1.9.1 is the notion of $\mathcal{O}^{\times}$-gerbe on $S_{\mathrm{dR}}$.

For a prestack $y$ we denote the corresponding 2-groupoid by $\mathrm{Ge}_{\mathcal{O}} \times\left(y_{\mathrm{dR}}\right)$.

Given a prestack $y$ and $\mathcal{G} \in \mathrm{Ge}_{\mathcal{O}} \times\left(y_{\mathrm{dR}}\right)$, we have a well-defined functor

$$
\text { Shvg }:\left(\text { PreStk }_{/ y}\right)^{\text {op }} \rightarrow \infty \text {-Cat. }
$$

If $\mathcal{L}$ is a line bundle on a prestack $y$ and $a$ is an element of $k / \mathbb{Z}$, the construction of [GR1, Example 6.4.6] defines an object $\mathcal{L}^{a} \in \mathrm{Ge}_{\mathcal{O}} \times\left(y_{\mathrm{dR}}\right)$. The assertion of Lemma 1.5.5 holds mutatis mutandis. The rest of the theory is unchanged.

1.9.4. For a finite type scheme $S$ we have a canonical map

$$
\mathrm{Ge}_{k \times, \text { tors }}(S) \rightarrow \mathrm{Ge}_{\mathcal{O}} \times\left(S_{\mathrm{dR}}\right) .
$$

It has the same properties as the map (1.12).

Note that in this case, $k^{\times \text {tors }}(-1)$ identifies with $\mathbb{Q} / \mathbb{Z}$, which we regard as a subgroup in $k / \mathbb{Z}$.

1.9.5. In addition to $\mathcal{O}^{\times}$-gerbes on $y_{\mathrm{dR}}$ for a scheme $y$, one can consider the notion of twisting on $y$ in the sense of [GR1, Sect. 6]. By definition, this is a $\mathcal{O}^{\times}$-gerbe on $y_{\mathrm{dR}}$, equipped with a trivialization of its pullback to $y$. We denote the space of twistings on $y$ by $\operatorname{Tw}(y)$.

Let $\mathcal{L}$ be again a line bundle on $y$, and let $\kappa$ be an element of $k$. To this data the construction of [GR1, Sect. 6] attaches an object $\mathcal{L}^{\kappa} \in \operatorname{Tw}(y)$. The image of $\mathcal{L}^{\kappa}$ under the tautological projection

$$
\mathrm{Tw}(y) \rightarrow \mathrm{Ge}_{\mathcal{O}} \times\left(y_{\mathrm{dR}}\right)
$$

is $\mathcal{L}^{a}$, where $a$ is the image of $\kappa$ under $k \rightarrow k / \mathbb{Z}$.

1.9.6. In what follows we will stay in the context of étale sheaves and gerbes, leaving it to the reader to make appropriate modifications for the other sheaf-theoretic contexts.

\section{Factorization gerbes on the afFine Grassmannian}

In this section we introduce our main object of study: factorization gerbes on the affine Grassmannian, which we stipulate to be the parameters for the metaplectic Langlands theory.

2.1. The Ran space. The Ran space of a curve $X$ is an algebro-geometric device (first suggested in [BD1]) that allows us to talk about factorization structures relative to our curve.

2.1.1. Let $X$ be a fixed smooth algebraic curve. We let Ran $\in$ PreStk be the Ran space of $X$. By definition, for an affine test scheme $S$, the $\operatorname{space} \operatorname{Maps}(S$, Ran) is discrete (i.e., is a set), and equals the set of finite non-empty subsets of the (set) $\operatorname{Maps}(S, X)$.

For a finite set $J$ we have a map

$$
\operatorname{Ran}^{J} \rightarrow \operatorname{Ran}
$$

given by the union of the corresponding finite subsets.

This operation makes Ran into a (non-unital) semi-group object in PreStk (see [Lu2, Definition 5.4.1.1] for what this means). 
2.1.2. The Ran space admits the following explicit description as a colimit (as an object of PreStk):

$$
\operatorname{Ran}=\underset{I}{\operatorname{colim}} X^{I},
$$

where $I$ runs through the category opposite to that of non-empty finite sets and surjective maps ${ }^{9}$. For a surjection $\phi: I_{1} \rightarrow I_{2}$, the corresponding map $X^{I_{2}} \rightarrow X^{I_{1}}$ is the corresponding diagonal morphism, denoted $\Delta_{\phi}$.

2.1.3. We denote by

$$
(\operatorname{Ran} \times \operatorname{Ran})_{\operatorname{disj}} \subset \operatorname{Ran} \times \operatorname{Ran}
$$

the open substack corresponding to the following condition:

For an affine test scheme $S$, and two points

$$
I_{1}, I_{2} \in \operatorname{Maps}(S, \operatorname{Ran}),
$$

the point $I_{1} \times I_{2} \in \operatorname{Maps}(S$, $\operatorname{Ran} \times \operatorname{Ran})$ belongs to $(\operatorname{Ran} \times \operatorname{Ran})_{\text {disj }}$ if the corresponding subsets

$$
I_{1}, I_{2} \subset \operatorname{Maps}(S, X)
$$

satisfy the following condition: for every $i_{1} \in I_{1}, i_{2} \in I_{2}$, the corresponding two maps $S \rightrightarrows X$ have non-intersecting images.

2.1.4. We give a similar definition for any power: for a finite set $J$ we let

$$
\operatorname{Ran}_{\text {disj }}^{J} \subset \operatorname{Ran}^{J}
$$

be the open substack corresponding to the following condition:

An $S$-point of $\operatorname{Ran}^{J}$, given by

$$
I_{j} \subset \operatorname{Maps}(S, X), \quad j \in J
$$

belongs to $\operatorname{Ran}_{\mathrm{disj}}^{J}$ if for every $j_{1} \neq j_{2}$ and $i_{1} \in I_{j_{1}}, i_{2} \in I_{j_{2}}$, the corresponding two maps $S \rightrightarrows X$ have non-intersecting images.

2.2. Factorization patterns over the Ran space. Let $Z$ be a prestack over Ran. At the level of $k$-points, a factorization structure on $Z$ is the following system of isomorphisms:

For a $k$-point $\underline{x}$ of Ran corresponding a finite set $x_{1}, \ldots, x_{n}$ of $k$-points of $X$, the fiber $Z_{\underline{x}}$ of $Z$ over the above point is supposed to be identified with

$$
\prod_{i} Z_{\left\{x_{i}\right\}}
$$

where $\left\{x_{i}\right\}$ are the corresponding singleton points of Ran.

We will now spell this idea, and some related notions, more precisely.

2.2.1. By a factorization structure on $Z$ we shall mean an assignment for any finite set $J$ of an isomorphism

$$
Z^{J} \underset{\operatorname{Ran}^{J}}{\times} \operatorname{Ran}_{\mathrm{disj}}^{J} \stackrel{\gamma_{J}}{\simeq} Z \underset{\operatorname{Ran}}{\times} \operatorname{Ran}_{\mathrm{disj}}^{J}
$$

where the morphism $\operatorname{Ran}^{J} \rightarrow$ Ran is given by (2.1).

We require the isomorphisms (2.2) to be compatible with surjections of finite sets in the sense that for $I \stackrel{\phi}{\rightarrow} J$ the diagram

${ }^{9}$ We note that this category is not filtered, and hence Ran is not an ind-scheme. 


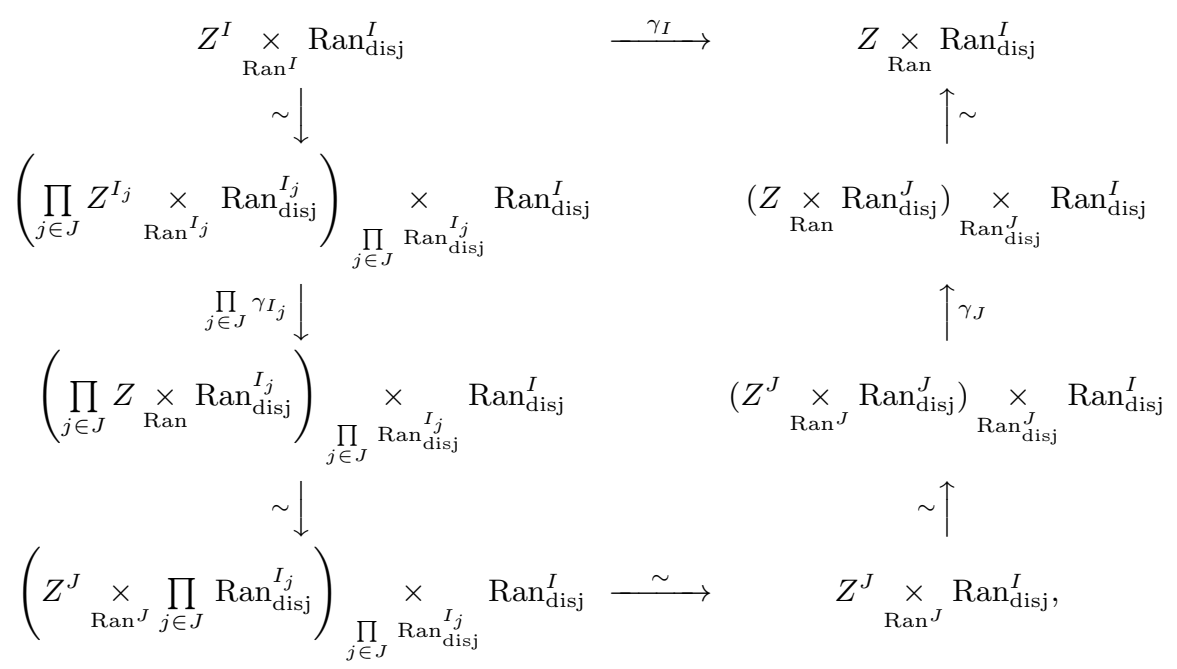

where $I_{j}:=\phi^{-1}(j)$, is required to commute. Furthermore, if $Z$ takes values in $\infty$-groupoids (rather than sets), we require a homotopy-coherent system of compatibilities for higher order compositions, see [Ras1, Sect. 6].

2.2.2. Let $\mathcal{C}$ be a presheaf of DG categories over Ran. By a pre-factorization structure on $\mathcal{C}$ we shall mean a functorial assignment for any finite set $J$ and an $S$-point of $\operatorname{Ran}_{\text {disj }}^{J}$, given by

$$
I_{j} \subset \operatorname{Maps}(S, X), \quad j \in J
$$

of a fully faithful functor

$$
\bigotimes_{j, \operatorname{Shv}(S)} \mathrm{C}\left(S, I_{j}\right) \rightarrow \mathfrak{C}(S, I)
$$

where $I=\sqcup_{j \in J} I_{j}$.

We require the functors (2.4) to be compatible with surjections $J_{1} \rightarrow J_{2}$ via the commutative diagrams analogous to (2.3). A precise formulation of these compatibilities is given in [Ras1, Sect. 6].

We will say that prefactorization structure on $\mathcal{C}$ is a factorization structure if the functors (2.4) are equivalences.

2.2.3. For example, let $Z$ be a factorization prestack over Ran. Then the presheaf of categories $\operatorname{Shv}(Z) /$ Ran, given by

$$
\operatorname{Shv}(Z) / \operatorname{Ran}:(S, I \subset \operatorname{Maps}(S, X)) \rightsquigarrow \operatorname{Shv}(S \underset{\operatorname{Ran}}{\times} Z),
$$

has a natural prefactorization structure.

Typically, this prefactorization structure is not a factorization structure, for the same reason as one given in Sect. 1.6.5.

2.2.4. Let $Z$ be a factorization prestack over Ran, and let $A$ be a torsion abelian group. Let $\mathcal{G}$ be an $A$-gerbe on $Z$. By a factorization structure on $\mathcal{G}$ we shall mean a system of identifications

$$
\left.\left.\mathcal{G}^{\bigotimes J}\right|_{Z^{J}} \underset{\operatorname{Ran} J}{\operatorname{Ran}_{\mathrm{disj}}^{J}} \simeq \mathcal{G}\right|_{Z \times \operatorname{Ran}_{\mathrm{disj}}^{J}},
$$

where the underlying spaces are identified via (2.2).

The identifications (2.5) are required to be compatible with surjections $J_{1} \rightarrow J_{2}$ via the commutative diagrams (2.3). Note that since gerbes form a 2-groupoid, we only need to specify the datum of (2.5) up to $|J|=3$, and check the relations up to $|J|=4$. 
Factorization gerbes over $Z$ naturally form a space (in fact, a 2-groupoid), equipped with a structure of commutative group in Spc (i.e., connective spectrum), to be $\operatorname{denoted} \operatorname{FactGe}_{A}(Z)$.

Remark 2.2.5. Note that the diagrams (2.2) include those corresponding to automorphisms of finite sets. I.e., the datum of factorization gerbe includes equivariance with respect to the action of the symmetric group. For this reason what we call "factorization gerbe" in [Re] was called "symmetric factorizable gerbe".

2.2.6. Let $Z$ be a factorization prestack over Ran, and let $\mathcal{G}$ be a factorization $E^{\times, \text {tors }}$-gerbe over it. Then the presheaf of categories $\operatorname{Shv}_{\mathcal{G}}(Z) /$ Ran defined by

$$
(S, I \subset \operatorname{Maps}(S, X)) \rightsquigarrow \operatorname{Shv}_{\mathcal{G}}(S \underset{\operatorname{Ran}}{\times} Z)
$$

is a sheaf of categories, and has a natural prefactorization structure.

2.2.7. By a similar token, we can consider factorization line bundles over factorization prestacks, and also $\mathbb{Z}$ - or $\mathbb{Z} / 2 \mathbb{Z}$-graded line bundles ${ }^{10}$.

If $\mathcal{L}$ is a (usual, i.e., not graded) factorization line bundle and $a \in A(-1)$, we obtain a factorization gerbe $\mathcal{L}^{a}$.

2.3. The Ran version of the affine Grassmannian. In this subsection we introduce the Ran version of the affine Grassmannian, which plays a crucial role in the geometric Langlands theory.

2.3.1. For an algebraic group $G$, we define the Ran version of the affine Grassmannian of $G$, denoted $\mathrm{Gr}_{G}$, to be the following prestack.

For an affine test scheme $S$, the groupoid (in fact, set) $\operatorname{Maps}\left(S, \operatorname{Gr}_{G}\right)$ consists of triples

$$
\left(I, \mathcal{P}_{G}, \alpha\right),
$$

where $I$ is an $S$-point of $\operatorname{Ran}, \mathcal{P}_{G}$ is a $G$-bundle on $S \times X$, and $\alpha$ is a trivialization of $\mathcal{P}_{G}$ over the open subset $U_{I} \subset S \times X$ equal to the complement of the union of the graphs of the maps $S \rightarrow X$ corresponding to the elements of $I \subset \operatorname{Maps}(S, X)$.

2.3.2. The basic feature of the prestack $\mathrm{Gr}_{G}$ is that it admits a natural factorization structure over Ran, obtained by gluing bundles.

Hence, for a torsion abelian group $A$, it makes sense to talk about factorization $A$-gerbes over $\mathrm{Gr}_{G}$. We denote the the resulting space (i.e., in fact, a connective 2-truncated spectrum) by

$$
\operatorname{FactGe}_{A}\left(\operatorname{Gr}_{G}\right) \text {. }
$$

2.3.3. An example. Let $\mathcal{L}$ be a factorization line bundle on $\operatorname{Gr}_{G}$, and let a be an element of $A(-1)$. Then the $A$-gerbe

$$
\mathcal{L}^{a}
$$

of Sect. 1.5.1 is naturally a factorization gerbe on $\mathrm{Gr}_{G}$.

This example is important because there is a canonical factorization line bundle on $\mathrm{Gr}_{G}$, denoted $\operatorname{det}_{G}$; we will encounter it in Sect. 5.2.1.

\footnotetext{
${ }^{10}$ Note that in the latter case, the compatibility involved in the factorization structure (arising from the diagrams (2.3) for automorphisms of finite sets $J$ ) involves sign rules. I.e., a factorization $\mathbb{Z} / 2 \mathbb{Z}$-graded line bundle does not give rise to a factorization line bundle by forgetting the grading.
} 
2.3.4. Assume for a moment that $X$ is proper.

Let $\operatorname{Bun}_{G}$ denote the moduli stack of $G$-bundles on $X$. Note that we have a tautological projection

$$
\mathrm{Gr}_{G} \rightarrow \operatorname{Bun}_{G} \text {. }
$$

Recall now that [GL2, Theorem 3.2.13] says $^{11}$ that the map (2.6) is a universal homological equivalence. This implies that any gerbe on $\mathrm{Gr}_{G}$ uniquely descends to a gerbe on $\mathrm{Bun}_{G}$.

In particular, this is the case for factorization gerbes.

\subsection{The space of geometric metaplectic data.}

2.4.1. We stipulate that the space

$$
\text { FactGe } E^{\times, \text {tors }}\left(\mathrm{Gr}_{G}\right)
$$

is the space of parameters for the metaplectic Langlands theory. We also refer to it as geometric metaplectic datum.

This includes both the global case (when $X$ is complete), and the local case when we take $X$ to be a Zariski neighborhood of some point $x$.

2.4.2. Given an $E^{\times \text {,tors }}$-factorization gerbe $\mathcal{G}$ on $\mathrm{Gr}_{G}$, we can thus talk about the prefactorization sheaf of categories, denoted

whose value on $S, I \subset \operatorname{Maps}(S, X)$ is

$$
\operatorname{Shv}_{\mathcal{G}}\left(\operatorname{Gr}_{G}\right) / \operatorname{Ran},
$$

$$
\operatorname{Shv}_{\mathcal{G}}\left(S \underset{\operatorname{Ran}}{\times} \operatorname{Gr}_{G}\right) .
$$

\section{Parameterization of factorization Gerbes}

From now on we let $A$ be a torsion abelian group whose elements have orders prime to $\operatorname{char}(k)$. The main example is $A=E^{\times \text {,tors }}$.

The goal of this section is to describe the set of isomorphism classes (and, more ambitiously, the space) of $A$-factorization gerbes on $\mathrm{Gr}_{G}$ in terms of more concise algebro-geometric objects.

3.1. Parameterization via étale cohomology. In this subsection we will create a space, provided by the theory of étale cohomology, that maps to the space $\operatorname{FactGe}_{E \times \text {,tors }}\left(\operatorname{Gr}_{G}\right)$, thereby giving a parameterization of geometric metaplectic data.

3.1.1. Let $B_{\text {et }}(G):=\mathrm{pt} / G$ be the stack of $G$-torsors. I.e., this is the sheafification in the étale topology of the prestack $B(G)$ that attaches to an affine test scheme $S$ the groupoid

$$
\text { */Maps }(S, G) \text {. }
$$

3.1.2. Consider the space of maps

$$
\operatorname{Maps}\left(B_{\text {et }}(G) \times X, B_{\text {et }}^{4}(A(1))\right),
$$

which is the same as $\operatorname{Maps}\left(B(G) \times X, B_{\text {et }}^{4}(A(1))\right)$.

We claim that there is a naturally defined map

$$
\operatorname{Maps}\left(B_{\text {et }}(G) \times X, B_{\text {et }}^{4}(A(1))\right) \underset{\operatorname{Maps}\left(X, B_{\mathrm{et}}^{4}(A(1))\right)}{\times} * \rightarrow \operatorname{FactGe}_{A}\left(\operatorname{Gr}_{G}\right),
$$

where the map

$$
\operatorname{Maps}\left(B_{\text {et }}(G) \times X, B_{\text {et }}^{4}(A(1))\right) \rightarrow \operatorname{Maps}\left(X, B_{\text {et }}^{4}(A(1))\right)
$$

corresponds to evaluation on the base point of $B_{\text {et }}(G)$.

\footnotetext{
${ }^{11}$ This assertion was proved in loc.cit. under the additional assumption that $G$ be semi-simple and simply connected. However, in the case of constant groups-schemes, the statement is known to hold in general: see [Ga3, Theorem 4.1.6].
} 
3.1.3. The construction of the map (3.1) proceeds as follows. Let us be given a map

$$
B_{\text {et }}(G) \times X \rightarrow B_{\text {et }}^{4}(A(1)),
$$

equipped with a trivialization of the composition

$$
X \rightarrow B_{\text {et }}(G) \times X \rightarrow B_{\text {et }}^{4}(A(1)) .
$$
$S$.

For an affine test scheme $S$ and an $S$-point $\left(I, \mathcal{P}_{G}, \alpha\right)$ of $\operatorname{Gr}_{G}$, we need to construct a $A$-gerbe $\mathcal{G}_{I}$ on

Moreover, for $\phi: I \rightarrow J$, such that the point

$$
\left\{\phi^{-1}(j) \subset \operatorname{Maps}\left(S, \operatorname{Ran}^{J}\right), \quad j \in J\right\}
$$

hits $\operatorname{Ran}_{\text {disj }}^{J}$, we need to be given an identification

$$
\mathcal{G}_{I} \simeq \bigotimes_{j \in J} \mathcal{G}_{I_{j}}
$$

3.1.4. Let us interpret the datum of $\mathcal{P}_{G}$ as a map

$$
S \times X \rightarrow B_{\text {et }}(G) \times X .
$$

Composing with (3.2), we obtain a map

$$
S \times X \rightarrow B_{\mathrm{et}}^{4}(A(1)),
$$

and a trivialization of the resulting map

$$
U_{I} \rightarrow B_{\mathrm{et}}^{4}(A(1))
$$

where $U_{I}$ is as in Sect. 2.3.1.

We claim that such a datum indeed gives rise to a $A$-gerbe $\mathcal{G}_{I}$ on $S$, equipped with identifications (3.3).

3.1.5. First off, since

$$
H_{\mathrm{et}}^{i}(S \times X, A(1)) \text { and } H_{\mathrm{et}}^{i-1}\left(U_{I}, A(1)\right)
$$

for $i=3$ and $i=4$ vanish étale-locally on $S$, we obtain that the prestack that sends $S$ to the space of maps (3.4), equipped with a trivialization of (3.5), identifies with $B_{\text {et }}^{2}$ of the prestack that sends $S$ to the space of maps

$$
S \times X \rightarrow B_{\mathrm{et}}^{2}(A(1))
$$

equipped with a trivialization of

$$
U_{I} \rightarrow B_{\mathrm{et}}^{2}(A(1))
$$

3.1.6. Thus, given a map (3.6), equipped with a trivialization of (3.7), we need to construct a locally constant map

$$
S \rightarrow A
$$

whose dependence on (3.6) and the trivialization of (3.7) respects the structure of commutative group on $A(1)$.

Let $\Gamma_{I}$ denote the complement of $U_{I}$ (the scheme structure on $\Gamma_{I}$ is irrelevant). We need to construct the trace map

$$
H_{\mathrm{et}, \Gamma_{I}}^{2}(S \times X, A(1)) \rightarrow H_{\mathrm{et}}^{0}(S, A)
$$

Consider the maps

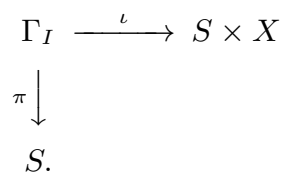


The map (3.8) follows by taking $H_{\mathrm{et}}^{0}(S,-)$ of the canonical map of sheaves

$$
\pi_{*} \circ \iota^{!}\left(A_{S \times X}\right)(1)[2] \rightarrow A_{S}
$$

where for a scheme $Y$, we denote by $A_{Y}$ the constant étale sheaf on $Y$ with value $A$.

3.1.7. In its turn, the map (3.9) is obtained by the $\left(\pi_{*}, \pi^{!}\right)$-adjunction from the isomorphism

$$
\iota^{!}\left(A_{S \times X}\right)(1)[2] \simeq \pi^{!}\left(A_{S}\right),
$$

where the latter comes from the identification

$$
\left(p_{X}\right) !\left(A_{\mathrm{pt}}\right) \simeq A_{X}(1)[2]
$$

where $p_{X}: X \rightarrow \mathrm{pt}$ is the projection.

\subsection{Analysis of homotopy groups of the space of factorization gerbes.}

3.2.1. We have the following assertion that results from [Re, Theorem II.7.3] and the computation of the homotopy groups of the left-hand side of (3.1) (the latter is given below):

Proposition 3.2.2. The map (3.1) is an isomorphism.

Remark 3.2.3. As was explained to us by J. Lurie, the assertion of Proposition 3.2.2 is nearly tautological if one works over the field of complex numbers and in the context of sheaves in the analytic topology.

3.2.4. From Proposition 3.2.2 we will obtain the following more explicit parameterization of the 2groupoid FactGe ${ }_{A}\left(\mathrm{Gr}_{G}\right)$.

Namely,

$$
\pi_{i}\left(\operatorname{FactGe}_{A}\left(\operatorname{Gr}_{G}\right)\right)=H_{\mathrm{et}}^{4-i}(B(G) \times X ; \mathrm{pt} \times X, A(1)) .
$$

Let us analyze what these cohomology groups look like. For the duration of this subsection we will assume that $A$ is divisible, unless $G$ is a torus.

3.2.5. Let $\pi_{1, \text { alg }}(G)$ denote the algebraic fundamental group of $G$. Explicitly, $\pi_{1, \text { alg }}(G)$ can be described as follows:

Choose a short exact sequence

$$
1 \rightarrow T_{2} \rightarrow \widetilde{G}_{1} \rightarrow G \rightarrow 1
$$

where $T_{2}$ is a torus and $\left[\widetilde{G}_{1}, \widetilde{G}_{1}\right]$ is simply connected. Set $T_{1}=\widetilde{G}_{1} /\left[\widetilde{G}_{1}, \widetilde{G}_{1}\right]$. Let $\Lambda_{1}$ and $\Lambda_{2}$ be the coweight lattices of $T_{1}$ and $T_{2}$, respectively. Then $\pi_{1, \text { alg }}(G) \simeq \Lambda_{1} / \Lambda_{2}$.

3.2.6. We have:

$$
\begin{gathered}
H_{\mathrm{et}}^{2 i+1}(B(G), A(1))=0 ; \\
H_{\mathrm{et}}^{2}(B(G), A(1)) \simeq \operatorname{Hom}\left(\pi_{1, \mathrm{alg}}(G), A\right) ; \\
H_{\mathrm{et}}^{4}(B(G), A(1)) \simeq \operatorname{Quad}(\Lambda, \mathbb{Z})^{W} \underset{\mathbb{Z}}{\otimes} A(-1),
\end{gathered}
$$

where $\operatorname{Quad}(\Lambda, \mathbb{Z})^{W}$ is the abelian group of $W$-invariant integer-valued quadratic forms on $\Lambda$.

Remark 3.2.7. We note that the natural map

$$
\operatorname{Quad}(\Lambda, \mathbb{Z})^{W} \underset{\mathbb{Z}}{\otimes} A(-1) \rightarrow \operatorname{Quad}(\Lambda, A(-1))^{W}
$$

is injective, but in general it is not surjective. 
3.2.8. By Künneth formula, we obtain:

$$
\pi_{0}\left(\operatorname{FactGe}_{A}\left(\operatorname{Gr}_{G}\right)\right)=\left(\operatorname{Quad}(\Lambda, \mathbb{Z})^{W} \underset{\mathbb{Z}}{\otimes} A(-1)\right) \times \operatorname{Hom}\left(\pi_{1, \operatorname{alg}}(G), A(-1)\right)
$$

if $X$ is proper, and just

$$
\operatorname{Quad}(\Lambda, \mathbb{Z})^{W} \underset{\mathbb{Z}}{\otimes} A(-1)
$$

otherwise.

Next,

$$
\pi_{1}\left(\operatorname{FactGe}_{A}\left(\operatorname{Gr}_{G}\right)\right)=H_{\mathrm{et}}^{1}\left(X, \operatorname{Hom}\left(\pi_{1, \operatorname{alg}}(G), A\right)\right) .
$$

Finally,

$$
\pi_{2}\left(\operatorname{FactGe}_{A}\left(\operatorname{Gr}_{G}\right)\right)=\operatorname{Hom}\left(\pi_{1, \operatorname{alg}}(G), A\right) .
$$

3.2.9. For a given $q \in \operatorname{Quad}(\Lambda, \mathbb{Z})^{W} \underset{\mathbb{Z}}{\otimes} A(-1) \subset \operatorname{Quad}(\Lambda, A(-1))^{W}$, let

$$
\text { FactGe }{ }_{A}^{q}\left(\operatorname{Gr}_{G}\right)
$$

denote the fiber of the map

$$
\operatorname{FactGe}_{A}\left(\operatorname{Gr}_{G}\right) \rightarrow \operatorname{Quad}(\Lambda, \mathbb{Z})^{W} \underset{\mathbb{Z}}{\otimes} A(-1)
$$

over $q$.

In particular, we can consider the commutative group in $\mathrm{Spc}$

$$
\text { Fact } \operatorname{Ge}_{A}^{0}\left(\operatorname{Gr}_{G}\right) \text {. }
$$

In Corollary 4.4.7 we will construct a canonical isomorphism:

$$
\operatorname{FactGe}_{A}^{0}\left(\operatorname{Gr}_{G}\right) \simeq \operatorname{Maps}\left(X, B_{\text {et }}^{2}\left(\operatorname{Hom}\left(\pi_{1, \operatorname{alg}}(G), A\right)\right)\right) \text {. }
$$

3.3. Parametrization of factorization line bundles. This subsection is included for the sake of completeness, in order to make contact with the theory of metaplectic extensions developed in [We].

Recall from Sect. 2.3.3 that given a factorization line bundle $\mathcal{L}$ on $\operatorname{Gr}_{G}$ and an element $a \in A(-1)$ we can produce a factorization gerbe $\mathcal{L}^{a}$. In this subsection we will describe a geometric data that gives rise to factorization line bundles ${ }^{12}$ on $\mathrm{Gr}_{G}$.

3.3.1. Let $K_{2}$ denote the prestack over $X$ that associates to an affine scheme $S=\operatorname{Spec}(A)$ mapping to $X$ the abelian group $K_{2}(A)$. Let $\left(K_{2}\right)$ Zar be the sheafification of $K_{2}$ in the Zariski topology.

On the one hand, we consider the space $\operatorname{CExt}\left(G,\left(K_{2}\right)\right.$ zar) (in fact, an ordinary groupoid) of Brylinski-Deligne data, which are by definition central extensions

$$
1 \rightarrow\left(K_{2}\right)_{\mathrm{Zar}} \rightarrow \widetilde{G} \rightarrow G \times X \rightarrow 1
$$

of the constant group-scheme $G \times X$ by $\left(K_{2}\right)$ zar .

The operation of Baer sum makes $\operatorname{CExt}\left(G,\left(K_{2}\right)\right.$ Zar $)$ into a commutative group in spaces, i.e., into a Picard category.

On the other hand, consider the Picard category

$$
\operatorname{FactPic}\left(\mathrm{Gr}_{G}\right)
$$

of factorizable line bundles on $\mathrm{Gr}_{G}$.

We are going to construct a map of Picard categories

$$
\operatorname{CExt}\left(G,\left(K_{2}\right) \text { Zar }\right) \rightarrow \operatorname{FactPic}\left(\operatorname{Gr}_{G}\right) .
$$

\footnotetext{
${ }^{12}$ We emphasize that this construction produces just factorization line bundles, and not $\mathbb{Z} / 2 \mathbb{Z}$-graded ones.
} 
3.3.2. The construction of the map (3.11) proceeds along the lines parallel to Sect. 3.1. We interpret the datum of an object of $\operatorname{CExt}\left(G,\left(K_{2}\right)_{\text {Zar }}\right)$ as a map

$$
B_{\mathrm{Zar}}(G) \times X \rightarrow B_{\mathrm{Zar}}^{2}\left(K_{2}\right),
$$

equipped with a trivialization of the composition

$$
X \rightarrow B_{\mathrm{Zar}}(G) \times X \rightarrow B_{\mathrm{Zar}}^{2}\left(K_{2}\right) .
$$

Given such a map, for an affine scheme $S$ and an $S$-point $\left(I, \mathcal{P}_{G}, \alpha\right)$ of $\mathrm{Gr}_{G}$, we need to construct a line bundle $\mathcal{L}_{I}$ on $S$. By [DrSi, Theorem 2], after passing to an étale cover of $S$, the $G$-bundle $\mathcal{P}_{G}$ becomes Zariski locally trivial. Hence, we can assume that $\left(I, \mathcal{P}_{G}, \alpha\right)$ is a map

$$
S \times X \rightarrow B_{\mathrm{Zar}}(G),
$$

equipped with a trivialization of the composition

$$
U_{I} \rightarrow S \times X \rightarrow B_{\mathrm{Zar}}(G),
$$

where $U_{I}$ is as in Sect. 2.3.1.

3.3.3. Composing (3.13) with (3.12) we obtain a map

$$
S \times X \rightarrow B_{\text {Zar }}^{2}\left(K_{2}\right)
$$

equipped with a trivialization of the composition

$$
U_{I} \rightarrow S \times X \rightarrow B_{\mathrm{Zar}}^{2}\left(K_{2}\right) .
$$

To this data we need to associate a line bundle $\mathcal{L}_{I}$ on $S$.

3.3.4. As in Sect. 3.1.5, it suffices to construct an invertible function on $S$, starting from the data of a map

$$
S \times X \rightarrow B_{\mathrm{Zar}}\left(K_{2}\right)
$$

equipped with a trivialization of the composition

$$
U_{I} \rightarrow S \times X \rightarrow B_{\mathrm{Zar}}\left(K_{2}\right) .
$$

The desired map comes from the residue map

$$
K_{2}\left(U_{I}\right) / K_{2}(S \times X) \rightarrow K_{1}(S) \simeq \Gamma\left(S, \mathcal{O}_{S}^{\times}\right),
$$

constructed as follows (Zariski sheafification is automatic since $\Gamma_{I}=S \times X-U_{I}$ is finite over $S$ ).

3.3.5. Consider the exact triangle of categories

$$
\operatorname{Perf}(S \times X)_{\Gamma_{I}} \rightarrow \operatorname{Perf}(S \times X) \rightarrow \operatorname{Perf}\left(U_{I}\right),
$$

where

$$
\operatorname{Perf}(S \times X)_{\Gamma_{I}} \subset \operatorname{Perf}(S \times X)
$$

is the full subcategory spanned by objects set-theoretically supported on $\Gamma_{I}$.

The long exact cohomology sequence gives rise to a map

$$
K_{2}\left(U_{I}\right) / K_{2}(S \times X) \rightarrow K_{1}\left(\operatorname{Perf}(S \times X)_{\Gamma_{I}}\right) .
$$

Now, the direct image functor

$$
\pi_{*}: \mathrm{QCoh}(S \times X) \rightarrow \mathrm{QCoh}(S)
$$

has the property that it sends $\operatorname{Perf}(S \times X)_{\Gamma_{I}}$ to $\operatorname{Perf}(S)$. Thus, we obtain a map

$$
K_{1}\left(\operatorname{Perf}(S \times X)_{\Gamma_{I}}\right) \rightarrow K_{1}(S) .
$$

Composing (3.20) and (3.21), we obtain the sought-for map (3.19). 
3.3.6. We propose:

Conjecture 3.3.7. The map (3.11), constructed above, is an isomorphism.

Remark 3.3.8. One can show that it follows from [BrDe, Theorem 3.16] combined with Sect. 4.1.5 that Conjecture 3.3.7 holds when $G=T$ is a torus.

3.4. Relationship between the two parameterizations. This subsection is also included for the sake of completeness; its contents will not be used in the sequel.

We will give a cohomological construction a map

$$
\operatorname{CExt}\left(G,\left(K_{2}\right) \text { Zar }\right) \rightarrow \operatorname{Maps}\left(B(G) \times X, B_{\mathrm{et}}^{4}\left(\mu_{n}(1)\right)\right) \underset{\operatorname{Maps}\left(X, B_{\mathrm{et}}^{4}\left(\mu_{n}(1)\right)\right)}{\times} *
$$

where the left-hand side is the space of Brylinski-Deligne data, and the right-hand side is the space parameterizing factorization gerbes on the affine Grassmannian.

3.4.1. Let $n$ be an integer invertible in $k$. Then the construction of [Sou] defines a map

$$
K_{2}(S) \rightarrow H_{\mathrm{et}}^{2}\left(S, \mu_{n}^{\otimes 2}\right),
$$

that depends functorially on $S \in \mathrm{Sch}^{\text {aff }}$.

As was explained to us by D. Clausen, the map (3.22) cannot be lifted to a map of commutative group objects in PreStk

$$
K_{2} \rightarrow B_{\text {et }}^{2}\left(\mu_{n}^{\otimes 2}\right) .
$$

3.4.2. Let us consider $B_{\text {et }}^{2}\left(\mu_{n}^{\otimes 2}\right)$ as a sheaf of commutative group objects in Spc on the big Zariski site. Let us consider its Postnikov truncation $\tau_{\leq 1}\left(B_{\text {et }}^{2}\left(\mu_{n}^{\otimes 2}\right)\right)$, again as a sheaf on the big Zariski site. We have the following fiber sequence

$$
B_{\text {Zar }}^{2}\left(\mu_{n}^{\otimes 2}\right) \rightarrow B_{\text {et }}^{2}\left(\mu_{n}^{\otimes 2}\right) \rightarrow \tau_{\leq 1}\left(B_{\text {et }}^{2}\left(\mu_{n}^{\otimes 2}\right)\right) .
$$

As was explained to us by A. Beilinson, the map (3.22) does lift to a map of presheaves

$$
K_{2} \rightarrow \tau_{\leq 1}\left(B_{\text {et }}^{2}\left(\mu_{n}^{\otimes 2}\right)\right) .
$$

The map (3.24) gives rise to a map

$$
B_{\text {Zar }}^{2}\left(K_{2}\right) \rightarrow \tau_{\leq 3}\left(B_{\text {et }}^{4}\left(\mu_{n}^{\otimes 2}\right)\right) .
$$

3.4.3. Note now that we have a fiber sequence

$$
B_{\text {Zar }}^{4}\left(\mu_{n}^{\otimes 2}\right) \rightarrow B_{\text {et }}^{4}\left(\mu_{n}^{\otimes 2}\right) \rightarrow \tau_{\leq 3}\left(B_{\text {et }}^{4}\left(\mu_{n}^{\otimes 2}\right)\right),
$$

from which it follows that the induced map

$$
\begin{aligned}
\operatorname{Maps}\left(B(G) \times X, B_{\text {et }}^{4}\left(\mu_{n}^{\otimes 2}\right)\right) \underset{\operatorname{Maps}\left(X, B_{\text {et }}^{4}\left(\mu_{n}^{\otimes 2}\right)\right)}{\times} & \\
& \rightarrow \operatorname{Maps}\left(B(G) \times X, \tau_{\leq 3}\left(B_{\text {et }}^{4}\left(\mu_{n}^{\otimes 2}\right)\right)\right) \underset{\operatorname{Maps}\left(X, \tau_{\leq 3}\left(B_{\text {et }}^{4}\left(\mu_{n}^{\otimes 2}\right)\right)\right)}{\times}
\end{aligned}
$$

is an isomorphism.

Combining with (3.25) we obtain a map

$$
\begin{aligned}
& \text { (3.26) } \operatorname{CExt}\left(G,\left(K_{2}\right) \text { Zar }\right) \simeq \operatorname{Maps}\left(B(G) \times X, B_{\text {Zar }}^{2}\left(K_{2}\right)\right) \underset{\operatorname{Maps}\left(X, B_{\text {Zar }}^{2}\left(K_{2}\right)\right)}{\times} * \rightarrow \\
& \rightarrow \operatorname{Maps}\left(B(G) \times X, B_{\text {et }}^{4}\left(\mu_{n}^{\otimes 2}\right)\right) \underset{\operatorname{Maps}\left(X, B_{\text {et }}^{4}\left(\mu_{n}^{\otimes 2}\right)\right)}{\times} *=\operatorname{Maps}\left(B(G) \times X, B_{\text {et }}^{4}\left(\mu_{n}(1)\right)\right) \underset{\operatorname{Maps}\left(X, B_{\text {et }}^{4}\left(\mu_{n}(1)\right)\right)}{\times}
\end{aligned}
$$

We propose: 
Conjecture 3.4.4. The following diagram commutes:

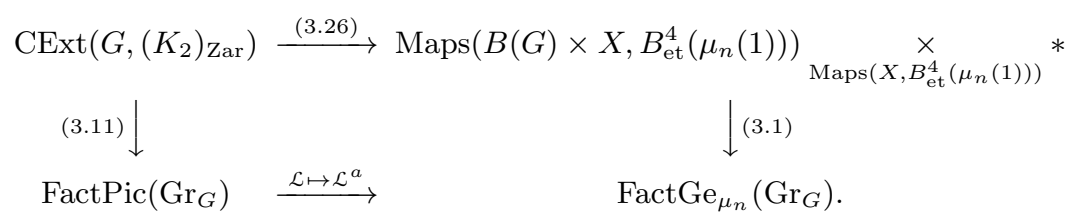

Remark 3.4.5. The assertion of Conjecture 3.4.4 should follow from the compatibility of the map (3.24) with the trace/symbol map.

\section{THE CASE OF TORI}

In this section we let $G=T$ be a torus. We will perform an explicit analysis of factorization gerbes on the affine Grassmannian $\mathrm{Gr}_{T}$, and introduce two related objects (multiplicative and commutative factorization gerbes) that would play an important role in the sequel.

4.1. Factorization Grassmannian for a torus. In this section we will show that the affine Grassmannian of a torus can be approximated by a prestack assembled from (=written as a colomit of) powers of $X$.

4.1.1. Recall that $\Lambda$ denotes the coweight lattice of $G=T$. Consider the index category whose objects are pairs $\left(I, \lambda^{I}\right)$, where $I$ is a finite non-empty set and $\lambda^{I}$ is a map $I \rightarrow \Lambda$; in what follows we will denote by $\lambda_{i} \in \Lambda$ is the value of $\lambda^{I}$ on $i \in I$.

A morphism $\left(J, \lambda^{J}\right) \rightarrow\left(I, \lambda^{I}\right)$ is a surjection $\phi: I \rightarrow J$ such that

$$
\lambda_{j}=\sum_{i \in \phi^{-1}(j)} \lambda_{i}
$$

Consider the prestack

$$
\operatorname{Gr}_{T, \mathrm{comb}}:=\underset{\left(I, \lambda^{I}\right)}{\operatorname{colim}} X^{I} .
$$

The prestack $\operatorname{Gr}_{T, \text { comb }}$ endowed with its natural forgetful map to Ran, also has a natural factorization structure.

There is a canonical map

$$
\mathrm{Gr}_{T, \mathrm{comb}} \rightarrow \mathrm{Gr}_{T}
$$

compatible with the factorization structures.

Namely, for each $\left(I, \lambda^{I}\right)$ the corresponding $T$-bundle on $X^{I} \times X$ is

$$
\bigotimes_{i \in I} \lambda_{i} \cdot \mathcal{O}\left(\Delta_{i}\right)
$$

where $\Delta_{i}$ is the divisor on $X^{I} \times X$ corresponding to the $i$-th coordinate being equal to the last one.

4.1.2. As in [Ga2, Sect. 8.1] one shows that the map (4.2) induces an isomorphism of the sheafifications in the topology generated by finite surjective maps. In particular, for any $S \rightarrow$ Ran, the map

$$
\mathrm{Ge}_{A}\left(S \underset{\operatorname{Ran}}{\times} \operatorname{Gr}_{T}\right) \rightarrow \mathrm{Ge}_{A}\left(S \underset{\operatorname{Ran}}{\times} \mathrm{Gr}_{T, \text { comb }}\right)
$$

is an isomorphism, and hence, so is the map

$$
\operatorname{FactGe}_{A}\left(\mathrm{Gr}_{T}\right) \rightarrow \operatorname{FactGe}_{A}\left(\mathrm{Gr}_{T, \mathrm{comb}}\right) .
$$

Furthermore, for a given $\mathcal{G} \in$ FactGe $_{E^{\times} \text {,tors }}\left(\operatorname{Gr}_{T}\right)$, the corresponding map of sheaves of categories

$$
\operatorname{Shv}_{\mathcal{G}}\left(\operatorname{Gr}_{T}\right) / \operatorname{Ran} \rightarrow \operatorname{Shv}{ }_{\mathcal{G}}\left(\operatorname{Gr}_{T, \text { comb }}\right) / \operatorname{Ran}
$$

is also an isomorphism. 
4.1.3. The datum of a factorization gerbe on $\mathrm{Gr}_{T \text {, comb }}$ can be explicitly described as follows:

For a finite set $I$ and a map

$$
\lambda^{I}: I \rightarrow \Lambda
$$

we specify a gerbe $\mathcal{G}_{\lambda^{I}}$ on $X^{I}$.

For a surjection of finite sets $I \stackrel{\phi}{\rightarrow} J$ such that (4.1) holds, we specify an identification

$$
\left(\Delta_{\phi}\right)^{*}\left(\mathcal{G}_{\lambda^{I}}\right) \simeq \mathcal{G}_{\lambda^{J}} .
$$

The identifications (4.3) must be compatible with compositions of maps of finite sets in the natural sense.

Let now $I \stackrel{\phi}{\rightarrow} J$ be a surjection of finite sets, and let

$$
X_{\phi, \operatorname{disj}}^{I} \subset X^{I}, \quad x_{i_{1}} \neq x_{i_{2}} \text { whenever } \phi\left(i_{1}\right) \neq \phi\left(i_{2}\right)
$$

be the corresponding open subset. For $j \in J$, let $\lambda^{I_{j}}$ be the restriction of $\lambda^{I}$ to $I_{j}$.

We impose the structure of factorization that consists of isomorphisms

$$
\left.\left.\left(\mathcal{G}_{\lambda^{I}}\right)\right|_{X_{\phi, \mathrm{disj}}^{I}} \simeq\left(\bigotimes_{j \in J} \mathcal{G}_{\lambda^{I_{j}}}\right)\right|_{X_{\phi, \mathrm{disj}}^{I}} .
$$

The isomorphisms (4.4) must be compatible with compositions of maps of finite sets in the natural sense.

In addition, the isomorphisms (4.4) and (4.3) must be compatible in the natural sense.

4.1.4. For a factorization gerbe $\mathcal{G}$ on $\operatorname{Gr}_{T, \text { comb }}$, the value of the category $\operatorname{Shv}_{\mathcal{G}}\left(\operatorname{Gr}_{T, \mathrm{comb}}\right) / \operatorname{Ran}$ on $X^{I}$ corresponding to a given $\lambda^{I}$ identifes with

$$
\operatorname{Shv}_{\mathcal{G}_{\lambda} I}\left(X^{I}\right) .
$$

This description implies that the sheaf of categories $\operatorname{Shv}_{\mathcal{G}}\left(\mathrm{Gr}_{T}\right)$ / Ran is quasi-coherent (see Sect. 1.6.5 for what this means), and that its prefactorization structure is actually a factorization structure.

Note that the corresponding facts would be false for a group $G$ that is not a torus.

4.1.5. The case of factorization line bundles. The datum of a factorization $\mathbb{Z} / 2 \mathbb{Z}$-graded line bundle on $\mathrm{Gr}_{T, \text { comb }}$ can be described in a way similar to that of factorization gerbes. This description recovers the notion of what in [BD1, Sect. 3.10.3] is called a $\theta$-datum.

We note that a factorization $\mathbb{Z} / 2 \mathbb{Z}$-graded line bundle is evenly (i.e., trivially) graded if and only if the corresponding $\theta$-datum is even, i.e., if the corresponding symmetric bilinear $\mathbb{Z}$-valued form on $\Lambda$ comes from a $\mathbb{Z}$-valued quadratic form.

We also note that [BD1, Proposition 3.10.7] says that restriction along

$$
\mathrm{Gr}_{T, \mathrm{comb}} \rightarrow \mathrm{Gr}_{T}
$$

defines an equivalence between the Picard categories of factorization ( $\mathbb{Z} / 2 \mathbb{Z}$-graded) line bundles.

4.2. Making the parameterization explicit for tori. In this subsection we will show explicitly how a multiplicative $A$-gerbe on $\mathrm{Gr}_{T}$ gives rise to an $A$-valued quadratic form

$$
q: \Lambda \rightarrow A(-1) .
$$


4.2.1. We first describe the bilinear form

$$
b: \Lambda \times \Lambda \rightarrow A(-1) .
$$

Given two elements $\lambda_{1}, \lambda_{2} \in \Lambda$, consider $I=\{1,2\}$ and the map

$$
\lambda^{I}: I \rightarrow \Lambda ; \quad 1 \mapsto \lambda_{1}, 2 \mapsto \lambda_{2}
$$

Consider the corresponding gerbe

$$
\mathcal{G}_{\lambda_{1}, \lambda_{2}}:=\mathcal{G}_{\lambda^{I}}
$$

over $X^{2}$. By (4.4) it is identified with $\mathcal{G}_{\lambda_{1}} \otimes \mathcal{G}_{\lambda_{2}}$ over $X^{2}-\Delta(X)$. By Lemma 1.5.5, there exists a well-defined element $a \in A(-1)$ such that

$$
\mathcal{G}_{\lambda_{1}, \lambda_{2}} \simeq\left(\mathcal{G}_{\lambda_{1}} \otimes \mathcal{G}_{\lambda_{2}}\right) \otimes \mathcal{O}(\Delta(X))^{a} .
$$

We let

$$
a=: b\left(\lambda_{1}, \lambda_{2}\right)
$$

4.2.2. It is easy to see that the resulting map

$$
b: \Lambda \times \Lambda \rightarrow A(-1)
$$

is symmetric. The fact that it is bilinear form can be seen as follows. For a triple of elements $\lambda_{1}, \lambda_{2}, \lambda_{3}$ consider the corresponding gerbes

over $X^{3}$.

$$
\mathcal{G}_{\lambda_{1}, \lambda_{2}, \lambda_{3}} \text { and }\left(\mathcal{G}_{\lambda_{1}, \lambda_{2}} \otimes \mathcal{G}_{\lambda_{3}}\right) \otimes \mathcal{O}\left(\Delta_{1,3}\right)^{\otimes B\left(\lambda_{1}, \lambda_{3}\right)} \otimes \mathcal{O}\left(\Delta_{2,3}\right)^{\otimes B\left(\lambda_{2}, \lambda_{3}\right)}
$$

They are identified away from the main diagonal $\Delta_{1,2,3}$, and hence this identification extends to all of $X^{3}$, since $\Delta_{1,2,3}$ has codimension 2 . Restricting to $\Delta_{1,2}$, we obtain an identification

$$
\mathcal{G}_{\lambda_{1}+\lambda_{2}, \lambda_{3}} \simeq\left(\mathcal{G}_{\lambda_{1}+\lambda_{2}} \otimes \mathcal{G}_{\lambda_{3}}\right) \otimes \mathcal{O}(\Delta)^{\otimes B\left(\lambda_{1}, \lambda_{3}\right)} \otimes \mathcal{O}(\Delta)^{\otimes B\left(\lambda_{2}, \lambda_{3}\right)}
$$

as gerbes over $X^{2}$. Comparing with the identification

we obtain the desired

$$
\mathcal{G}_{\lambda_{1}+\lambda_{2}, \lambda_{3}} \simeq\left(\mathcal{G}_{\lambda_{1}+\lambda_{2}} \otimes \mathcal{G}_{\lambda_{3}}\right) \otimes \mathcal{O}(\Delta)^{\otimes b\left(\lambda_{1}+\lambda_{2}, \lambda_{3}\right)},
$$

$$
b\left(\lambda_{1}, \lambda_{3}\right)+b\left(\lambda_{2}, \lambda_{3}\right)=b\left(\lambda_{1}+\lambda_{2}, \lambda_{3}\right) .
$$

4.2.3. Finally, let us recover the quadratic form

$$
q: \Lambda \rightarrow A(-1) .
$$

For a given $\lambda \in \Lambda$, consider the gerbes $\mathcal{G}_{\lambda, \lambda}$ and $\mathcal{G}_{\lambda} \otimes \mathcal{G}_{\lambda}$ on $X^{2}$. They are both equipped with a structure of $S_{2}$-equivariance, and they are identified as such over $X^{2}-\Delta$. In addition, the induced equivariance structure on both

$$
\left.\mathcal{G}_{\lambda, \lambda}\right|_{\Delta} \text { and }\left(\mathcal{G}_{\lambda} \otimes \mathcal{G}_{\lambda}\right)_{\Delta}
$$

is the tautological one.

We note that the datum of a gerbe on $X^{2}$, equipped with a structure of $S_{2}$-equivariance, whose restriction to $\Delta$ is the tautological equivariance structure is equivalent to the datum of a gerbe on $X^{(2)}$, where the latter is the symmetric square of $X$. Hence, we obtain a well-define gerbe $\mathcal{G}_{\lambda^{(2)}}$ over $X^{(2)}$, trivialized away from the diagonal, so that

$$
\left.\mathcal{G}_{\lambda, \lambda} \simeq\left(\mathcal{G}_{\lambda} \otimes \mathcal{G}_{\lambda}\right) \otimes \mathcal{G}_{\lambda(2)}\right|_{X \times X}
$$

compatibly with the trivializations on $X^{2}-\Delta$.

By Lemma 1.5.5, $\mathcal{G}$ is canonically of the form $\mathcal{O}\left(\Delta^{\prime}\right)^{a^{\prime}}$ for some $a^{\prime} \in A(-1)$, and where $\Delta^{\prime}$ denotes the diagonal in $X^{(2)}$.

Set

$$
a^{\prime}=: q(\lambda) .
$$

By construction,

$$
\left.\mathcal{G}_{\lambda^{(2)}}\right|_{X \times X} \simeq(\mathcal{O}(\Delta))^{b(\lambda, \lambda)}
$$


compatibly with the trivializations on $X^{2}-\Delta$.

Since $\left.\mathcal{O}\left(\Delta^{\prime}\right)\right|_{X^{2}}=\mathcal{O}(\Delta)^{\otimes 2}$, we have

$$
b(\lambda, \lambda)=2 \cdot q(\lambda)
$$

The relation

$$
q\left(\lambda_{1}+\lambda_{2}\right)=q\left(\lambda_{1}\right)+q\left(\lambda_{2}\right)+b\left(\lambda_{1}, \lambda_{2}\right)
$$

is checked in a way similar to Sect. 4.2.2.

4.3. The notion of multiplicative/commutative factorization gerbe. In order to be able to state the metaplectic version of geometric Satake, we will need to discuss the notion of multiplicative/commutative factorization gerbe, first on $\mathrm{Gr}_{T}$, and then when the lattice $\Lambda=\operatorname{Hom}\left(\mathbb{G}_{m}, T\right)$ is replaced by a general finitely generated abelian group.

4.3.1. Note that since $T$ is commutative, $\mathrm{Gr}_{T}$ is naturally a (commutative) group-prestack over Ran. Hence, along with $\operatorname{FactGe}_{A}\left(\mathrm{Gr}_{T}\right)$, we can consider the corresponding spaces (in fact, commutative groups in spaces)

$$
\text { FactGe }{ }_{A}^{\text {mult }}\left(\operatorname{Gr}_{T}\right) \text { and } \text { FactGe }_{A}^{\text {com }}\left(\operatorname{Gr}_{T}\right)
$$

that correspond to gerbes that respect the group (resp., commutative group structure) on $\mathrm{Gr}_{T}$ over Ran.

We have the evident forgetful maps

$$
\operatorname{FactGe}_{A}^{\text {com }}\left(\mathrm{Gr}_{T}\right) \rightarrow \operatorname{FactGe}_{A}^{\text {mult }}\left(\mathrm{Gr}_{T}\right) \rightarrow \operatorname{FactGe} A\left(\mathrm{Gr}_{T}\right) .
$$

4.3.2. Let us regard $B(T)$ and $B_{\text {et }}^{4}(A)$ as (commutative) group objects in PreStk. From Proposition 3.2.2, we obtain:

\section{Corollary 4.3.3.}

(a) We have a canonical isomorphism

$$
\operatorname{Maps}_{\operatorname{Grp}\left(\operatorname{PreStk}_{X}\right)}\left(B(T) \times X, B_{\mathrm{et}}^{4}(A(1)) \times X\right) \simeq \operatorname{FactGe}_{A}^{\mathrm{mult}}\left(\operatorname{Gr}_{T}\right) .
$$

(b) We have a canonical isomorphism

$$
\operatorname{Maps}_{\left.\operatorname{ComGrp} \operatorname{PreStk}_{/ X}\right)}\left(B(T) \times X, B_{\mathrm{et}}^{4}(A(1)) \times X\right) \simeq \operatorname{FactGe}_{A}^{\mathrm{com}}\left(\mathrm{Gr}_{T}\right) .
$$

In the above corollary, the notation $\operatorname{Grp}(-)$ (resp., ComGrp $(-)$ ) means group-objects (resp., commutative group-objects) in a given $\infty$-category.

We are going to use Corollary 4.3.3 to describe the spaces

$$
\text { FactGe }_{A}^{\text {mult }}\left(\mathrm{Gr}_{T}\right) \text { and FactGe }{ }_{A}^{\text {com }}\left(\mathrm{Gr}_{T}\right)
$$

more explicitly.

4.3.4. Note that the Kummer map

$$
A \times \mathbb{G}_{m} \rightarrow B_{\text {et }}(A(1)),
$$

which is a map of commutative group-prestacks, gives rise to a map

$$
\operatorname{Hom}(\Lambda, A) \times T \rightarrow B_{\text {et }}(A(1))
$$

and hence

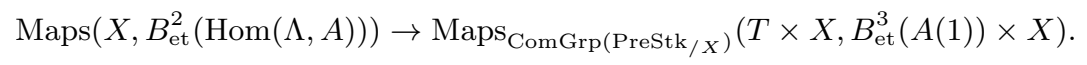

We also note that the looping map

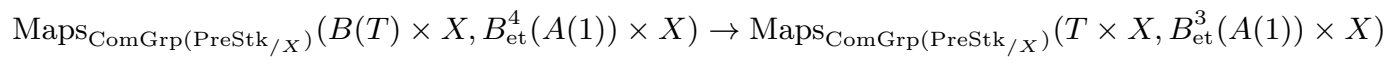

is an isomorphism.

Combining with Corollary 4.3.3(b), we obtain a map

$$
\operatorname{Maps}\left(X, B_{\text {et }}^{2}(\operatorname{Hom}(\Lambda, A))\right) \rightarrow \operatorname{FactGe}_{A}^{\text {com }}\left(\operatorname{Gr}_{T}\right) .
$$


We claim:

Corollary 4.3.5. The map (4.9) is an isomorphism.

The proof follows from the description of factorization gerbes in Sect. 4.1, or alternatively from Sect. 4.3.9 below.

4.3.6. We now claim:

Proposition 4.3.7. The forgetful maps

$$
\operatorname{FactGe}_{A}^{\text {com }}\left(\mathrm{Gr}_{T}\right) \rightarrow \operatorname{FactGe}_{A}^{\text {mult }}\left(\mathrm{Gr}_{T}\right) \rightarrow \operatorname{FactGe}_{A}\left(\mathrm{Gr}_{T}\right)
$$

are fully faithful. Moreover:

(a) The essential image of the composition

$$
\operatorname{FactGe}_{A}^{\text {com }}\left(\mathrm{Gr}_{T}\right) \rightarrow \operatorname{FactGe}_{A}^{\text {mult }}\left(\mathrm{Gr}_{T}\right) \rightarrow \operatorname{FactGe}_{A}\left(\mathrm{Gr}_{T}\right)
$$

equals FactGe $_{A}^{0}\left(\mathrm{Gr}_{T}\right)$.

(b) The essential image of

$$
\operatorname{FactGe}_{A}^{\text {mult }}\left(\mathrm{Gr}_{T}\right) \rightarrow \text { FactGe }_{A}\left(\mathrm{Gr}_{T}\right)
$$

is the preimage of the subset of $\operatorname{Quad}(\Lambda, A)$, consisting of those quadratic forms, whose associated bilinear form is zero.

Proof. Follows from Corollary 4.3.3, combined with the following lemma:

\section{Lemma 4.3.8.}

(a) $H_{\mathrm{et}}^{i}\left(B^{2}(T), A\right)=0$ for $i=1,2,4$. The map

$$
H_{\mathrm{et}}^{3}\left(B^{2}(T), A\right) \rightarrow H_{\mathrm{et}}^{2}(B(T), A) \simeq \operatorname{Hom}(\Lambda, A(-1))
$$

is an isomorphism. The map

$$
H_{\mathrm{et}}^{5}\left(B^{2}(T), A\right) \rightarrow H_{\mathrm{et}}^{4}(B(T), A) \simeq \operatorname{Quad}(\Lambda, A(-2))
$$

is injective and has as its image the set of quadratic forms whose associated bilinear form vanishes.

(b) For $k \geq 2$, we have $H_{\mathrm{et}}^{i+k}\left(B^{1+k}(T), A\right)=0$ for all positive integers $i$ up to $4+k$ except $i=2$. The natural map

is an isomorphism.

$$
H_{\mathrm{et}}^{2+k}\left(B^{1+k}(T), A\right) \rightarrow H_{\mathrm{et}}^{2}(B(T), A) \simeq \operatorname{Hom}(\Lambda, A(-1))
$$

4.3.9. Let us compute the homotopy groups of the spaces (4.5). We obtain:

$$
\pi_{0}\left(\operatorname{FactGe}_{A}^{\mathrm{mult}}\left(\mathrm{Gr}_{T}\right)\right) \simeq \operatorname{Hom}(\Lambda, A(-1))_{2 \text {-tors }} \times \operatorname{Hom}(\Lambda, A(-1))
$$

if $X$ is proper, and just $\operatorname{Hom}(\Lambda, A(-1))_{2}$-tors otherwise. Similarly,

$$
\pi_{0}\left(\operatorname{FactGe}_{A}^{\mathrm{com}}\left(\operatorname{Gr}_{T}\right)\right) \simeq \operatorname{Hom}(\Lambda, A(-1))
$$

if $X$ is proper, and 0 otherwise.

Next,

$$
\pi_{1}\left(\operatorname{FactGe}_{A}^{\text {mult }}\left(\operatorname{Gr}_{T}\right)\right) \simeq \pi_{1}\left(\operatorname{FactGe}_{A}^{\operatorname{com}}\left(\operatorname{Gr}_{T}\right)\right) \simeq H_{\mathrm{et}}^{1}(X, \operatorname{Hom}(\Lambda, A))
$$

Finally

$$
\pi_{2}\left(\operatorname{FactGe}_{A}^{\text {mult }}\left(\operatorname{Gr}_{T}\right)\right) \simeq \pi_{2}\left(\operatorname{FactGe}_{A}^{\operatorname{com}}\left(\operatorname{Gr}_{T}\right)\right) \simeq \operatorname{Hom}(\Lambda, A) .
$$

4.4. More general abelian groups. In this section we generalize the discussion of Sect. 4.3 to the case when instead of a lattice $\Lambda$ (thought of as a lattice of cocharacters of a torus) we take a general finitely generated abelian group.

We need this in order to state the metaplectic version of geometric Satake. 
4.4.1. Let $\Gamma$ be a finitely generated abelian group. We define the commutative group-prestack over Ran

$$
\mathrm{Gr}_{\Gamma \otimes \mathbb{G}_{m}}
$$

as follows. Write $\Gamma$ as $\Lambda_{1} / \Lambda_{2}$, where $\Lambda_{1} \supset \Lambda_{2}$ are lattices. Let $T_{1}$ and $T_{2}$ be the corresponding tori. We define $\operatorname{Gr}_{\Gamma \otimes \mathbb{G}_{m}}$ as a quotient of $\mathrm{Gr}_{T_{1}}$ by $\mathrm{Gr}_{T_{2}}$, viewed as commutative group-prestacks over Ran.

It is easy to see that this definition (as well as other constructions we are going to perform) is canonically independent of the presentation of $\Gamma$ as a quotient.

The group-prestack $\operatorname{Gr}_{\Gamma \otimes \mathbb{G}_{m}}$ has a natural factorization structure over Ran.

4.4.2. Let now $G$ be a connective reductive group. Let $\Gamma=\pi_{1, \text { alg }}(G)$. The description in Sect. 3.2.5 implies that there is a canonically defined map

$$
\mathrm{Gr}_{G} \rightarrow \mathrm{Gr}_{\Gamma \otimes \mathbb{G}_{m}}
$$

compatible with the factorization structure.

4.4.3. Since $\operatorname{Gr}_{\Gamma \otimes \mathbb{G}_{m}}$ is a commutative group-prestack over Ran, along with FactGe $\operatorname{Fe}_{A}\left(\operatorname{Gr}_{\Gamma \otimes \mathbb{G}_{m}}\right)$, we can consider the corresponding spaces (in fact, commutative groups in spaces)

$$
\operatorname{FactGe}_{A}^{\text {mult }}\left(\operatorname{Gr}_{\Gamma \otimes \mathbb{G}_{m}}\right) \text { and } \operatorname{FactGe}_{A}^{\mathrm{com}}\left(\operatorname{Gr}_{\Gamma \otimes \mathbb{G}_{m}}\right)
$$

that correspond to gerbes that respect that group (resp., commutative group structure) on $\operatorname{Gr}_{\Gamma \otimes \mathbb{G}_{m}}$ over Ran.

The following results from Proposition 4.3.7:

Corollary 4.4.4. Let $\Gamma$ be written as a quotient of two lattices as in Sect. 4.4.1. Let $\mathcal{G}_{1}$ be a factorization A-gerbe on $\mathrm{Gr}_{T_{1}}$, and let $b_{1}$ and $q_{1}$ be the associated bilinear and quadratic forms on $\Lambda_{1}$, respectively.

Then the datum of descent of the gerbe $\mathcal{G}_{1}$ to a factorization gerbe $\mathcal{G}$ on $\operatorname{Gr}_{\Gamma \otimes \mathbb{G}_{m}}$ exists only if the restriction of $q_{1}$ to $\Lambda_{2}$ is trivial, and in the latter case is equivalent to the trivialization of $\mathcal{G}_{2}:=\mathcal{G}_{1} \mid \mathrm{Gr}_{T_{2}}$ as a factorization gerbe on $\mathrm{Gr}_{T_{2}}$. Moreover:

(a) The gerbe $\mathcal{G}$ admits a multiplicative structure if and only if $b_{1}$ is trivial. In the latter case, the multiplicative structure is unique up to a unique isomorphism.

(b) The gerbe $\mathcal{G}$ admits a commutative multiplicative structure if and only if $q_{1}$ is trivial. In the latter case, the commutative multiplicative structure is unique up to a unique isomorphism.

From here we obtain:

\section{Corollary 4.4.5.}

(a) There is a canonical equivalence

$$
\operatorname{Maps}\left(X, B_{\mathrm{et}}^{2}(\operatorname{Hom}(\Gamma, A))\right) \simeq \operatorname{FactGe}_{A}^{\operatorname{com}}\left(\operatorname{Gr}_{\Gamma \otimes \mathbb{G}_{m}}\right) .
$$

(b) There is a diagram of fiber sequences

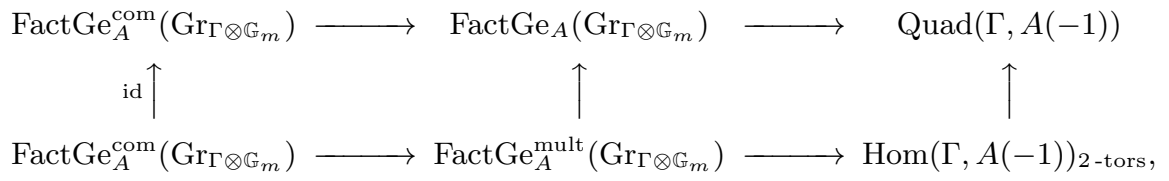

where $\operatorname{Hom}(\Lambda, A(-1))_{2 \text {-tors }}$ is identified with the kernel of the map

$$
\operatorname{Quad}(\Gamma, A(-1)) \rightarrow \operatorname{Bilin}(\Gamma, A(-1))
$$


4.4.6. Let now $\Gamma$ be the algebraic fundamental group $\pi_{1, \text { alg }}(G)$ of a reductive group $G$, and recall the $\operatorname{map}(4.10)$

Consider the composite map

$$
\operatorname{Gr}_{G} \rightarrow \operatorname{Gr}_{\pi_{1, \text { alg }}(G) \otimes \mathbb{G}_{m}}
$$

$$
\operatorname{FactGe}_{A}^{\mathrm{com}}\left(\operatorname{Gr}_{\pi_{1, \text { alg }}(G) \otimes \mathbb{G}_{m}}\right) \rightarrow \operatorname{FactGe}_{A}\left(\operatorname{Gr}_{\pi_{1, \mathrm{alg}}(G) \otimes \mathbb{G}_{m}}\right) \rightarrow \operatorname{FactGe}_{A}\left(\operatorname{Gr}_{G}\right) .
$$

From Corollary 4.4.5 and the calculation of homotopy groups in Sect. 3.2 .8 we obtain:

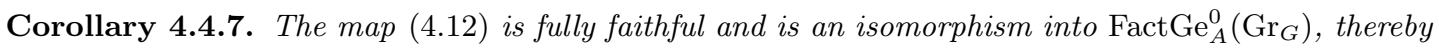
inducing an isomorphism

$$
\operatorname{Maps}\left(X, B_{\text {et }}^{2}\left(\operatorname{Hom}\left(\pi_{1, \operatorname{alg}}(G), A\right)\right)\right) \simeq \operatorname{FactGe}_{A}^{0}\left(\operatorname{Gr}_{G}\right) .
$$

4.5. Splitting multiplicative gerbes. In this subsection we will assume that $\operatorname{char}(k) \neq 2$. We will need to perform one more manipulation: it turns out that the fiber sequence

$$
\operatorname{FactGe}_{A}^{\mathrm{com}}\left(\operatorname{Gr}_{\Gamma \otimes \mathbb{G}_{m}}\right) \rightarrow \operatorname{FactGe}_{A}^{\text {mult }}\left(\operatorname{Gr}_{\Gamma \otimes \mathbb{G}_{m}}\right) \rightarrow \operatorname{Hom}(\Gamma, A(-1))_{2 \text {-tors }}
$$

of Corollary 4.4.5(b) admits a canonical splitting.

4.5.1. Note that

$$
\operatorname{Hom}(\Gamma, A(-1))_{2 \text {-tors }} \simeq \operatorname{Hom}\left(\Gamma, A(-1)_{2 \text {-tors }}\right) \simeq \operatorname{Hom}\left(\Gamma, A_{2 \text {-tors }}\right),
$$

since the group $\mu_{2}$ is canonically $\pm 1=\mathbb{Z} / 2 \mathbb{Z}$.

In order to define the sought-for splitting, by functoriality, it suffices to consider the case of $\Gamma=\mathbb{Z} / 2 \mathbb{Z}$, $A= \pm 1$ and we need to produce a multiplicative factorization gerbe on $\mathrm{Gr}_{\mathbb{Z} / 2 \mathbb{Z} \otimes \mathbb{G}_{m}}$ that gives rise to the tautological map $\mathbb{Z} / 2 \mathbb{Z} \rightarrow \pm 1$.

4.5.2. We will first construct the corresponding multiplicative factorization gerbe on $\operatorname{Gr}_{\mathbb{G}_{m}}$, i.e., for $\Gamma=\mathbb{Z}$. It will be clear from the construction that its pullback under the isogeny

$$
\mathbb{Z} \stackrel{2 \cdot-}{\longrightarrow} \mathbb{Z}
$$

is canonically trivial. This will give rise to the sought-for gerbe for $\Gamma=\mathbb{Z} / 2 \mathbb{Z}$ by Corollary $4.4 .4(\mathrm{a})$.

4.5.3. In order to perform the construction we will choose a datum of a $\mathbb{Z} / 2 \mathbb{Z}$-graded factorization line bundle $\mathcal{L}$ on $\operatorname{Gr}_{\mathbb{G}_{m}}$, Ran .

We require that the restriction of $\mathcal{L}$ to $X \subset$ Ran be such that its further restriction to the connected component of

$$
\operatorname{Gr}_{\mathbb{G}_{m}, X}:=X \underset{\operatorname{Ran}}{\times} \operatorname{Gr}_{\mathbb{G}_{m}}
$$

corresponding to $1 \in \mathbb{Z}$, is odd. An example of such an $\mathcal{L}$ is the determinant line bundle, corresponding to the tautological action of $\mathbb{G}_{m}$ on a 1-dimensional vector space.

We now consider the line bundle $\mathcal{L}^{\otimes 2}$, and the \pm 1 -gerbe $\left(\mathcal{L}^{\otimes 2}\right)^{\frac{1}{2}}$ (see Remark 1.5.3 for our notational convention). By unwinding the construction of the quadratic form in Sect. 4.2.3, it is easy to see that this factorization gerbe has the required property.

4.5.4. We now claim that the gerbe $\left(\mathcal{L}^{\otimes 2}\right)^{\frac{1}{2}}$ is canonically independent of the choice of $\mathcal{L}$. Indeed, let $\mathcal{L}_{1}$ and $\mathcal{L}_{2}$ be two different choices for $\mathcal{L}$. We note that their ratio $\widetilde{\mathcal{L}}:=\mathcal{L}_{1} \otimes \mathcal{L}_{2}^{\otimes-1}$ is a usual factorization line bundle (i.e., it is $\mathbb{Z} / 2 \mathbb{Z}$-graded, but the grading is even). So, the gerbe

$$
\left(\widetilde{\mathcal{L}}^{\otimes 2}\right)^{\frac{1}{2}}
$$

is canonically trivialized by means of the line bundle $\left(\widetilde{\mathcal{L}}^{\otimes 2}\right)^{\otimes \frac{1}{2}}=\widetilde{\mathcal{L}}$.

Remark 4.5.5. We note that, by construction, the gerbe $\left(\mathcal{L}^{\otimes 2}\right)^{\frac{1}{2}}$ admits a canonical trivialization. But this factorization is not compactible with the factorization structure.

4.5.6. In what follows, for a given element $\epsilon \in \operatorname{Hom}(\Gamma, A(-1))_{2 \text {-tors }}$, we will denote by $\mathcal{G}^{\epsilon}$ the resulting multiplicative factorization gerbe on $\operatorname{Gr}_{\Gamma \otimes \mathbb{G}_{m}}$. 
4.5.7. For a given object $\mathcal{G} \in \operatorname{FactGe}_{A}^{\text {mult }}\left(\operatorname{Gr}_{\Gamma \otimes \mathbb{G}_{m}}\right)$ let us denote by $\epsilon$ the map

$$
\Gamma \rightarrow A(-1)_{2 \text {-tors }}
$$

that measures the obstruction of $\mathcal{G}$ to belong to $\operatorname{FactGe}_{A}^{\text {com }}\left(\operatorname{Gr}_{\Gamma \otimes \mathbb{G}_{m}}\right)$.

We obtain that, canonically attached to $\mathcal{G}$, there exists an object

$$
\mathcal{G}^{\text {com }} \in \operatorname{FactGe}_{A}^{\text {com }}\left(\operatorname{Gr}_{\Gamma \otimes \mathbb{G}_{m}}\right),
$$

such that

where $\mathcal{G}^{\epsilon}$ is as in Sect. 4.5.6.

$$
\mathcal{G} \simeq \mathcal{G}^{\mathrm{com}} \otimes \mathcal{G}^{\epsilon}
$$

\section{JACQUET FUNCTORS FOR FACTORIZATION GERBES}

In this section we take $G$ to be reductive. We will study the interaction between factorization gerbes on $\mathrm{Gr}_{G}$ and those on $\mathrm{Gr}_{M}$, where $M$ is the Levi quotient of a parabolic of $G$.

5.1. The naive Jacquet functor. Let $P$ be a parabolic subgroup of $G$, and we let $P \rightarrow M$ be its Levi quotient. Let $N_{P}$ denote the unipotent radical of $P$.

5.1.1. Consider the diagram of the Grassmannians

$$
\mathrm{Gr}_{G} \stackrel{\mathrm{p}}{\longleftarrow} \mathrm{Gr}_{P} \stackrel{\mathrm{q}}{\longrightarrow} \mathrm{Gr}_{M} .
$$

We claim that pullback along q defines an equivalence,

$$
\operatorname{Ge}_{A}\left(S \underset{\operatorname{Ran}}{\times} \operatorname{Gr}_{M}\right) \rightarrow \operatorname{Ge}_{A}\left(S \underset{\operatorname{Ran}}{\times} \operatorname{Gr}_{P}\right)
$$

for any $S \rightarrow$ Ran, in particular, inducing an equivalence

$$
\operatorname{FactGe}_{A}\left(\operatorname{Gr}_{M}\right) \rightarrow \operatorname{FactGe}_{A}\left(\mathrm{Gr}_{P}\right) .
$$

5.1.2. To show that (5.1) is an equivalence, let us choose a splitting $M \hookrightarrow P$ of the projection $P \rightarrow M$. In particular, we obtain an adjoint action of $M$ on $N_{P}$. Hence, we obtain an action of the group-prestack $\mathfrak{L}^{+}(M)$ (see Sect. 7.1.3 for the definition of this group-prestack) over Ran on $\operatorname{Gr}_{N_{P}}$.

We can view $\operatorname{Gr}_{M}$ as a quotient $\mathfrak{L}(M) / \mathfrak{L}^{+}(M)$ (see Sect. 7.2.2), and hence we can view the map

$$
\mathfrak{L}(M) \rightarrow \mathrm{Gr}_{M}
$$

as a $\mathfrak{L}^{+}(M)$-torsor. Then $\mathrm{Gr}_{P}$, when viewed as a prestack over $\mathrm{Gr}_{M}$ is obtained by twisting $\mathrm{Gr}_{N_{P}}$ by the above $\mathfrak{L}^{+}(M)$-torsor.

Now, the equivalence in (5.1) follows from the fact that for any $S \rightarrow$ Ran, pullback defines an isomorphism

for all $i$.

$$
H_{\mathrm{et}}^{i}(S, A) \rightarrow H_{\mathrm{et}}^{i}\left(S \underset{\operatorname{Ran}}{\times} \mathrm{Gr}_{N_{P}}, A\right)
$$

5.1.3. In terms of the parameterization given by Proposition 3.2.2, the map

$$
\operatorname{FactGe}_{A}\left(\operatorname{Gr}_{G}\right) \rightarrow \operatorname{FactGe}_{A}\left(\operatorname{Gr}_{M}\right)
$$

can be interpreted as follows:

It corresponds to the map

$$
\begin{aligned}
& \operatorname{Maps}\left(B(G) \times X, B_{\mathrm{et}}^{4}(A(1))\right) \underset{\operatorname{Maps}\left(X, B_{\mathrm{et}}^{4}(A(1))\right)}{\times} * \rightarrow \\
\rightarrow & \operatorname{Maps}\left(B(P) \times X, B_{\mathrm{et}}^{4}(A(1))\right) \underset{\operatorname{Maps}\left(X, B_{\mathrm{et}}^{4}(A(1))\right)}{\times} \stackrel{\sim}{\sim} \operatorname{Maps}\left(B(M) \times X, B_{\mathrm{et}}^{4}(A(1))\right) \underset{\operatorname{Maps}\left(X, B_{\mathrm{et}}^{4}(A(1))\right)}{\times} *,
\end{aligned}
$$

where the second arrow is an isomorphism since the map $B(P) \rightarrow B(M)$ induces an isomorphism an étale cohomology with constant coefficients. 
Thus, if $\mathcal{G}^{G}$ is a factorization $A$-gerbe on $\mathrm{Gr}_{G}$, and $\mathcal{G}^{M}$ is the corresponding the factorization $A$-gerbe on $\mathrm{Gr}_{M}$, the corresponding quadratic forms

$$
q: \Lambda \rightarrow A(-1)
$$

coincide.

5.1.4. We now take $A:=E^{\times \text {,tors }}$. Given a factorization $E^{\times \text {,tors }}$-gerbe $\mathcal{G}^{G}$ over $\operatorname{Gr}_{G}$, consider its pullback to $\operatorname{Gr}_{P}$, denoted $\mathcal{G}^{P}$. We let $\mathcal{G}^{M}$ denote the canonically defined factorization gerbe on $\mathrm{Gr}_{M}$, whose pullback to $\operatorname{Gr}_{P}$ gives $\mathcal{G}^{P}$.

By construction, for any $S \rightarrow$ Ran, we have a well-defined pullback functor

$$
\mathrm{p}^{!}: \operatorname{Shv}_{\mathfrak{P}_{G}}\left(S \underset{\operatorname{Ran}}{\times} \operatorname{Gr}_{G}\right) \rightarrow{\operatorname{Shv} \mathcal{P}_{P}}_{P}\left(S \underset{\operatorname{Ran}}{\times} \operatorname{Gr}_{P}\right) .
$$

Furthermore, since the morphism $\mathrm{q}$ is ind-schematic, we have a well-defined push-forward functor

$$
\mathbf{q}_{*}: \operatorname{Shv}_{\mathcal{G}^{P}}\left(S \underset{\operatorname{Ran}}{\times} \operatorname{Gr}_{P}\right) \rightarrow \operatorname{Shv}_{\mathcal{G}^{M}}\left(S \underset{\operatorname{Ran}^{\times}}{\times} \operatorname{Gr}_{M}\right) .
$$

Thus, the composite $\mathbf{q}_{*} \circ \mathrm{p}^{!}$defines a map between prefactorization sheaves of categories

$$
\operatorname{Shv}_{\mathcal{G}^{G}}\left(\operatorname{Gr}_{G}\right)_{/ \text {Ran }} \rightarrow \operatorname{Shv}_{\mathcal{G}^{M}}\left(\operatorname{Gr}_{M}\right) / \operatorname{Ran} .
$$

We will refer to (5.2) as the naive Jacquet functor.

5.2. The critical twist. The functor (5.2) is not quite what we need for the purposes of geometric Satake. Namely, we will need to correct this functor by a cohomological shift that depends on the connected component of $\mathrm{Gr}_{M}$ (this is needed in order to arrange that the corresponding functor on the spherical categories maps perverse sheaves to perverse sheaves). However, this cohomological shift will destroy the compatibility of the Jacquet functor with factorization, due to sign rules. In order to compensate for this, we will apply an additional twist of our categories by the square root of the determinant line bundle.

The nature of this additional twist will be explained in the present subsection.

For the rest of this subsection we will assume that $\operatorname{char}(k) \neq 2$.

5.2.1. Let $\operatorname{det}_{G}$ denote the determinant line bundle on $\mathrm{Gr}_{G}$, corresponding to the adjoint representation. It is constructed as follows. For an affine test scheme $S$ and an $S$-point $I \subset \operatorname{Maps}(S, X)$ of Ran, consider the corresponding $G$-bundle $\mathcal{P}_{G}$ on $S \times X$, equipped with an isomorphism

$$
\alpha: \mathcal{P}_{G} \simeq \mathcal{P}_{G}^{0}
$$

over $U_{I} \subset S \times X$. Consider the corresponding vector bundles associated with the adjoint representation

$$
\left.\left.\mathfrak{g}_{\mathcal{P}_{G}}\right|_{U_{I}} \simeq \mathfrak{g}_{\mathcal{P}_{G}^{0}}\right|_{U_{I}}
$$

Then

$$
\operatorname{det} \text { rel. }\left(\mathfrak{g}_{\mathcal{P}_{G}}, \mathfrak{g}_{\mathcal{P}_{G}^{0}}\right)
$$

is a well-defined line bundle ${ }^{13}$ on $S$.

This construction is compatible with pullbacks under $S^{\prime} \rightarrow S$, thereby giving rise to the sought-for line bundle $\operatorname{det}_{G}$ on $\operatorname{Gr}_{G}$.

It is easy to see that $\operatorname{det}_{G}$ is equipped with a factorization structure over Ran.

\footnotetext{
${ }^{13}$ Note that the line bundle (5.3) is a priori $\mathbb{Z}$-graded, but since $G$ is reductive, and in particular, unimodular, this grading is actually trivial (i.e., concentrated in degree 0 ).
} 
5.2.2. Taking $A= \pm 1$, we will consider the factorization gerbe $\operatorname{det}_{G}^{\frac{1}{2}}$ over $\operatorname{Gr}_{G}$.

From now on we will choose a square root, denoted $\omega_{X}^{\otimes \frac{1}{2}}$ of the canonical line bundle $\omega_{X}$ on $X$ (see again Remark 1.5.3 for our notational conventions).

Let $P$ be again a parabolic of $G$. Consider the factorization gerbes $\left.\operatorname{det}_{G}^{\frac{1}{2}}\right|_{\operatorname{Gr}_{P}}$ and $\left.\operatorname{det}_{M}^{\frac{1}{2}}\right|_{\operatorname{Gr}_{P}}$ over $\operatorname{Gr}_{P}$. We claim that the choice of $\omega_{X}^{\otimes \frac{1}{2}}$ gives rise to an identification of gerbes

$$
\left.\left.\operatorname{det}_{G}^{\frac{1}{2}}\right|_{\operatorname{Gr}_{P}} \simeq \operatorname{det}_{M}^{\frac{1}{2}}\right|_{\operatorname{Gr}_{P}} \text {. }
$$

However, this identification will be compatible with the factorization structures only up to a sign.

In fact, we claim that the ratio of the line bundles

$$
\left.\operatorname{det}_{G}\right|_{\operatorname{Gr}_{P}} \text { and }\left.\operatorname{det}_{M}\right|_{\operatorname{Gr}_{P}}
$$

admits a square root, to be $\operatorname{denoted}_{\operatorname{det}_{P}}^{\otimes \frac{1}{2}}$, which is a $\mathbb{Z}$-graded (and, in particular, $\mathbb{Z} / 2 \mathbb{Z}$-graded) factorization line bundle on $\operatorname{Gr}_{P}$. The $\mathbb{Z} / 2 \mathbb{Z}$-grading on $\operatorname{det}_{P}^{\otimes \frac{1}{2}}$ is responsible for the sign defect in the identification of the gerbes (5.4).

Remark 5.2.3. In fact, more is true: the construction of [BD2, Sect. 4] defines a square root of $\operatorname{det}_{G}$ itself, again viewed as a factorization graded line bundle. This allows to trivialize the gerbe $\operatorname{det}_{G}^{\frac{1}{2}}$. But again, this trivialization is compatible with the factorization structure only up to a sign.

5.2.4. The graded line bundle $\operatorname{det}_{P}^{\otimes \frac{1}{2}}$ is constructed as follows. For an $S$-point $\left(I, \mathcal{P}_{P},\left.\left.\mathcal{P}_{G}\right|_{U_{I}} \simeq \mathcal{P}_{G}^{0}\right|_{U_{I}}\right)$ of $\operatorname{Gr}_{P}$ we set the value of $\operatorname{det}_{P}^{\otimes \frac{1}{2}}$ on $S$ to be

$$
\text { rel. det. }\left(\mathfrak{n}(P)_{\mathcal{P}_{P}}, \mathfrak{n}(P)_{\mathcal{P}_{P}^{0}}\right) \text {. }
$$

Let us construct the isomorphism

$$
\left.\left.\left(\operatorname{det}_{P}^{\otimes \frac{1}{2}}\right)^{\otimes 2} \otimes \operatorname{det}_{M}\right|_{\operatorname{Gr}_{P}} \simeq \operatorname{det}_{G}\right|_{\operatorname{Gr}_{P}} .
$$

Let us identify the vector space $\mathfrak{g} / \mathfrak{p}$ with the dual of $\mathfrak{n}(P)$ (say, using the Killing form). For an $S$-point $\left(I, \mathcal{P}_{P},\left.\left.\mathcal{P}_{G}\right|_{U_{I}} \simeq \mathcal{P}_{G}^{0}\right|_{U_{I}}\right)$ of $\mathrm{Gr}_{P}$, denote

$$
\mathcal{E}:=\mathfrak{n}(P)_{\mathcal{P}_{P}} \text { and } \mathcal{E}_{0}:=\mathfrak{n}(P)_{\mathcal{P}_{P}^{0}} .
$$

Then the ratio of $\left.\operatorname{det}_{G}\right|_{S}$ and $\left.\operatorname{det}_{M}\right|_{S}$ identifies with the line bundle

$$
\text { rel. det. }\left(\mathcal{E}, \mathcal{E}_{0}\right) \otimes \operatorname{rel} \text {. det. }\left(\mathcal{E}^{\vee}, \mathcal{E}_{0}^{\vee}\right) \text {. }
$$

Note, however, that for any line bundle $\mathcal{L}$ on $S \times X$, we have

$$
\text { rel. det. }\left(\mathcal{E}, \mathcal{E}_{0}\right) \otimes \text { rel. det. }\left(\mathcal{E}^{\vee}, \mathcal{E}_{0}^{\vee}\right) \simeq \text { rel. det. }\left(\mathcal{E} \otimes \mathcal{L}, \mathcal{E}_{0} \otimes \mathcal{L}\right) \otimes \operatorname{rel} \text {. det. }\left(\mathcal{E}^{\vee} \otimes \mathcal{L}, \mathcal{E}_{0}^{\vee} \otimes \mathcal{L}\right) \text {. }
$$

Letting $\mathcal{L}$ be the pullback of $\omega_{X}^{\otimes \frac{1}{2}}$, we thus need to construct an isomorphism

$$
\text { rel. det. }\left(\mathcal{E} \otimes \omega_{X}^{\otimes \frac{1}{2}}, \mathcal{E}_{0} \otimes \omega_{X}^{\otimes \frac{1}{2}}\right) \simeq \text { rel. det. }\left(\mathcal{E}^{\vee} \otimes \omega_{X}^{\otimes \frac{1}{2}}, \mathcal{E}_{0}^{\vee} \otimes \omega_{X}^{\otimes \frac{1}{2}}\right) .
$$

However, this follows from the (relative to $S$ ) local Serre duality on $S \times X$ :

$$
\mathbb{D}_{/ S}^{\text {Serre }}\left(\mathcal{E} \otimes \omega_{X}^{\otimes \frac{1}{2}}\right) \simeq \mathcal{E}^{\vee} \otimes \omega_{X}^{\otimes \frac{1}{2}}[1] \text { and } \mathbb{D}_{/ S}^{\text {Serre }}\left(\mathcal{E}_{0} \otimes \omega_{X}^{\otimes \frac{1}{2}}\right) \simeq \mathcal{E}_{0}^{\vee} \otimes \omega_{X}^{\otimes \frac{1}{2}}[1]
$$

5.3. The corrected Jacquet functor. We will now use the square root gerbe $\operatorname{det}_{P}^{\otimes \frac{1}{2}}$ from the previous subsection in order to introduce a correction to the naive Jacquet functor from Sect. 5.1.4.

5.3.1. Let $\Lambda_{M /[M, M]}$ be cocharacter lattice of the torus $M /[M, M]$. We have a canonical map from the set of connected components of $\operatorname{Gr}_{M /[M, M]}$ (and hence also $\operatorname{Gr}_{M}$ and $\operatorname{Gr}_{P}$ ) to $\Lambda_{M /[M, M]}$. Let $2 \check{\rho}_{G, M}$ be the character of $M /[M, M]$ equal to the determinant of the action of $M$ on $\mathfrak{n}(P)$.

Coupling with $2 \check{\rho}_{G, M}$ we obtain a (locally constant map)

$$
d_{G, M}: \mathrm{Gr}_{M} \rightarrow \mathbb{Z}
$$


5.3.2. Given a factorization $E^{\times \text {,tors }}$-gerbe $\mathcal{G}^{G}$ on $\operatorname{Gr}_{G}$ and the corresponding factorization gerbe $\mathcal{G}^{M}$ on $\mathrm{Gr}_{M}$ (see Sect. 5.1.4), we will now define the corrected Jacquet functor as a map between prefactorization sheaves of categories:

$$
J_{M}^{G}: \operatorname{Shv}_{\mathcal{G}^{G} \otimes \operatorname{det}_{G}^{\frac{1}{2}}}\left(\operatorname{Gr}_{G}\right) / \operatorname{Ran} \rightarrow \operatorname{Shv}_{\mathcal{G}^{M} \otimes \operatorname{det}_{M}^{\frac{1}{2}}}\left(\operatorname{Gr}_{M}\right) / \operatorname{Ran} .
$$

Namely, for an affine test scheme $S$ and an $S$-point of Ran, the corresponding functor is the composition of the following four operations:

(i) The pullback functor

$$
\mathrm{p}^{!}: \operatorname{Shv}_{\mathcal{P}_{G} \otimes \operatorname{det}_{G}^{\frac{1}{2}}}\left(S \underset{\operatorname{Ran}}{\times} \operatorname{Gr}_{G}\right) \rightarrow \operatorname{Shv}_{\mathcal{P}_{G} \otimes \operatorname{det}_{G}^{\frac{1}{2}}}\left(S \underset{\operatorname{Ran}}{\times} \operatorname{Gr}_{P}\right) ;
$$

(ii) The identification

$$
\operatorname{Shv}_{\mathcal{P}_{G} \otimes \operatorname{det}_{G}^{\frac{1}{2}}}\left(S \underset{\operatorname{Ran}}{\times} \operatorname{Gr}_{P}\right) \simeq \operatorname{Shv}_{\mathcal{G}^{M} \otimes \operatorname{det}_{M}^{\frac{1}{2}}}\left(S \underset{\operatorname{Ran}}{\times} \operatorname{Gr}_{P}\right),
$$

given by the isomorphism of gerbes (5.4);

(iii) The pushforward functor

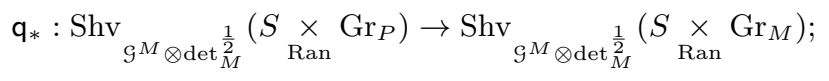

(iv) The cohomological shift by $\left[-d_{G, M}\right]$ (depending on the connected component of $\operatorname{Gr}_{M}$ ).

5.3.3. We claim that the functor (5.5) is a functor between factorization categories. This follows from the fact that the natural grading on the line bundle $\operatorname{det}_{P}^{\otimes \frac{1}{2}}$ is such that it equals $d_{G, M}$ on the corresponding connected component of $\mathrm{Gr}_{P}$.

\section{The metaplectic Langlands dual Datum}

In section we take $G$ to be reductive. Given a factorization gerbe $\mathcal{G}$ on $\operatorname{Gr}_{G}$, we will define the metaplectic Langlands dual datum attached to $\mathcal{G}$, and the corresponding notion of twisted local system on $X$.

6.1. The metaplectic Langlands dual root datum. The first component of the metaplectic Langlands dual datum is purely combinatorial and consists of a certain root datum that only depends on the root datum of $G$ and $q$. This is essentially the same as the root datum defined by G. Lusztig as a recipient of the quantum Frobenius.

6.1.1. Given a factorization $A$-gerbe $\mathcal{G}^{G}$ on $\mathrm{Gr}_{G}$, let

$$
\begin{gathered}
q: \Lambda \rightarrow A(-1) \\
b: \Lambda \times \Lambda \rightarrow A(-1)
\end{gathered}
$$

be the associated quadratic and bilinear forms, respectively. Let $\Lambda^{\sharp} \subset \Lambda$ be the kernel of $b$.

Following [Lus], we will now define a new root datum

$$
\left(\Delta^{\sharp} \subset \Lambda^{\sharp}, \check{\Delta}^{\sharp} \subset \check{\Lambda}^{\sharp}\right) .
$$


6.1.2. We let $\Delta^{\sharp}$ be equal to $\Delta$ as an abstract set. For each element $\alpha \in \Delta$, we let the corresponding element $\alpha^{\sharp} \in \Delta^{\sharp}$ be equal to

$$
\operatorname{ord}(q(\alpha)) \cdot \alpha \in \Lambda
$$

and the corresponding element $\check{\alpha}^{\sharp} \in \check{\Delta}^{\sharp}$ be

$$
\frac{1}{\operatorname{ord}(q(\alpha))} \cdot \check{\alpha} \in \check{\Lambda} \underset{\mathbb{Z}}{\otimes} \mathbb{Q} .
$$

The fact that $q \in \operatorname{Quad}(\Lambda, A(-1))$ lies in the image of the map

$$
\operatorname{Quad}(\Lambda, \mathbb{Z})^{W} \underset{\mathbb{Z}}{\otimes} A(-1) \rightarrow \operatorname{Quad}(\Lambda, A(-1))^{W}
$$

(see Remark 3.2.7) implies that $\alpha^{\sharp}$ and $\check{\alpha}^{\sharp}$ defined in this way indeed belong to $\Lambda^{\sharp} \subset \Lambda$ and $\check{\Lambda}^{\sharp} \subset \check{\Lambda} \underset{\mathbb{Z}}{\otimes} \mathbb{Q}$, respectively.

6.1.3. Since $q$ was $W$-invariant, the action of $W$ on $\Lambda$ preserves $\Lambda^{\sharp}$. Moreover, for each $\alpha \in \Delta$, the action of the corresponding reflection $s_{\alpha} \in W$ on $\Lambda^{\sharp}$ equals that of $s_{\alpha^{\sharp}}$.

This implies that restriction defines an isomorphism from $W$ to the group $W^{\sharp}$ of automorphisms of $\Lambda^{\sharp}$ generated by the elements $s_{\alpha \sharp}$.

Hence, (6.1) is a finite root system with Weyl group $W^{\sharp}$, isomorphic to the original Weyl group $W$.

It follows from the constriction that if $\alpha_{i}$ are the simple coroots of $\Delta$, then the corresponding elements $\alpha_{i}^{\sharp} \in \Lambda^{\sharp}$ form a set of simple roots of $\Delta^{\sharp}$.

6.1.4. We let $G^{\sharp}$ denote the reductive group (over $k$ ) corresponding to (6.1).

6.2. The " $\pi_{1}$-gerbe". Let $\mathcal{G}^{G}$ be as above. In this subsection we will show that in addition to the reductive group $G^{\sharp}$, the datum of $\mathcal{G}^{G}$ defines a certain multiplicative factorization gerbe on the affine Grassmannian corresponding to the abelian group $\pi_{1, \text { alg }}\left(G^{\sharp}\right)$.

6.2.1. Let $\mathcal{G}^{T}$ be the factorization gerbe on $\mathrm{Gr}_{T}$, corresponding to $\mathcal{G}^{G}$ via Sect. 5.1.4. Consider the corresponding torus $T^{\sharp}$.

Let $\mathcal{G}^{T^{\sharp}}$ be the factorization gerbe on $\mathrm{Gr}_{T^{\sharp}}$, Ran equal to the pullback of $\mathcal{G}^{T}$ under $T^{\sharp} \rightarrow T$. By Proposition 4.3.7(b), the gerbe $\mathcal{G}^{T^{\sharp}}$ carries a canonical multiplicative structure.

Consider the algebraic fundamental group $\pi_{1, \text { alg }}\left(G^{\sharp}\right)$ of $G^{\sharp}$, and the projection $\Lambda^{\sharp} \rightarrow \pi_{1, \text { alg }}\left(G^{\sharp}\right)$. Consider the corresponding map

$$
\mathrm{Gr}_{T^{\sharp}} \rightarrow \mathrm{Gr}_{\pi_{1, \mathrm{alg}}\left(G^{\sharp}\right) \otimes \mathbb{G}_{m}} .
$$

We claim that there exists a canonically defined multiplicative factorization $A$-gerbe $\mathcal{G}^{\pi_{1, \text { alg }}\left(G^{\sharp}\right) \otimes \mathbb{G}_{m}}$ on $\operatorname{Gr}_{\pi_{1, \text { alg }}\left(G^{\sharp}\right) \otimes \mathbb{G}_{m}}$, whose pullback under (6.2) identifies with $\mathcal{G}^{T^{\sharp}}$.

6.2.2. By Corollary 4.4.4, we need to show that for every simple coroot $\alpha_{i}$, the pullback of $\mathcal{G}^{T}$ to $\operatorname{Gr}_{\mathbb{G}_{m}}$ under

$$
\mathbb{G}_{m} \stackrel{\alpha_{i}^{\sharp}}{\longrightarrow} T
$$

is naturally trivialized.

By the transitivity of the construction in Sect. 5.1.4, we can replace $G$ by its Levi subgroup $M_{i}$ of semi-simple rank 1, corresponding to $\alpha_{i}$. Furthermore, using the map $S L_{2} \rightarrow M_{i}$, we can thus assume that $G=S L_{2}$. 
6.2.3. Note that by Sect. 3.2.8, any factorizable $A$-gerbe on $\operatorname{Gr}_{S L_{2}}$ is canonically of the form $\left(\operatorname{det}_{S L_{2}}\right)^{a}$ for some element $a \in A(-1)$.

Let us first calculate the resulting $A$-gerbe on $\mathrm{Gr}_{\mathbb{G}_{m}}$, where we think of $\mathbb{G}_{m}$ as the Cartan subgroup of $S L_{2}$.

For an integer $k$ let $\operatorname{det}_{\mathbb{G}_{m}, k}$ denote the determinant line bundle on $\operatorname{Gr}_{\mathbb{G}_{m}}$ associated with the action of $\mathbb{G}_{m}$ on the one-dimensional vector space given by the $k$-th power of the tautological character. This a $\mathbb{Z}$-graded factorization line bundle, and we note that the grading is even if $k$ is even.

By Sect. 5.2.4, the factorization gerbe on $\operatorname{Gr}_{\mathbb{G}_{m}}$, corresponding to $\left(\operatorname{det}_{S L_{2}}\right)^{a}$ is given by $\left(\operatorname{det}_{\mathbb{G}_{m}, 2}\right)^{2 a}$. The associated quadratic form

$$
q: \mathbb{Z} \rightarrow A
$$

takes value $4 a$ on the generator $1 \in \mathbb{Z}$. Let $n:=\operatorname{ord}(4 a)$.

We need to show that the pullback of $\left(\operatorname{det}_{\mathbb{G}_{m}, 2}\right)^{2 a}$ under the isogeny

$$
\mathbb{G}_{m} \stackrel{x \mapsto x^{n}}{\longrightarrow} \mathbb{G}_{m}
$$

is canonically trivial as a factorization gerbe on $\mathrm{Gr}_{\mathbb{G}_{m}, \operatorname{Ran}}$.

6.2.4. Note that the pullback of $\operatorname{det}_{\mathbb{G}_{m}, 2}$ under the above isogeny is the factorization line bundle $\operatorname{det}_{\mathbb{G}_{m}, 2 n}$. We need to provide a canonical trivialization of the factorization gerbe

$$
\left(\operatorname{det}_{\mathbb{G}_{m}, 2 n}\right)^{2 a} \text {. }
$$

For that it is sufficient to show that the factorization line bundle $\operatorname{det}_{\mathbb{G}_{m}, 2 n}$ on $\operatorname{Gr}_{\mathbb{G}_{m}}$ admits a canonical $2 n$-th root.

6.2.5. Note that, as in Corollary 4.3.5 (see also [BD1, Lemma 3.10.3]), a line bundle on $X$ gives rise to a factorization line bundle on $\mathrm{Gr}_{\mathbb{G}_{m}}$. Denote this construction by

$$
\mathcal{L} \mapsto \operatorname{Fact}(\mathcal{L}) .
$$

Now, we have:

$$
\operatorname{det}_{\mathbb{G}_{m}, 2 n} \simeq\left(\left(\operatorname{det}_{\mathbb{G}_{m}, 1}\right)^{\otimes 2 n}\right)^{\otimes 2 n} \otimes\left(\operatorname{Fact}\left(\omega_{X}^{\otimes \frac{2 n-1}{2}}\right)\right)^{\otimes 2 n} .
$$

Thus, the line bundle $\left(\operatorname{det}_{\mathbb{G}_{m}, 1}\right)^{\otimes 2 n} \otimes \operatorname{Fact}\left(\omega_{X}^{\otimes \frac{2 n-1}{2}}\right)$ gives the desired $2 n$-th root.

6.2.6. Example. Suppose that $\mathcal{G}^{G}$ is trivial, in which case $T^{\sharp}=T$ and $G^{\sharp}=G$. In this case $\mathcal{G}^{\pi_{1, \mathrm{alg}}\left(G^{\sharp}\right) \otimes \mathbb{G}_{m}}$ is also trivial.

6.3. The metaplectic Langlands dual datum as a triple. Until the end of this section we will assume that $\operatorname{char}(k) \neq 2$. We take $A$ to be $E^{\times \text {,tors }}$.

6.3.1. By Sect. 4.5 , to $\mathcal{G}^{\pi_{1, \text { alg }} \otimes \mathbb{G}_{m}}$ we can canonically attach a commutative factorization gerbe $\left(\mathcal{G}^{\pi_{1, \mathrm{alg}} \otimes \mathbb{G}_{m}}\right)^{\mathrm{com}}$ on $\mathrm{Gr}_{\pi_{1, \mathrm{alg}}\left(G^{\sharp}\right) \otimes \mathbb{G}_{m}}$, and a map

$$
\epsilon: \pi_{1, \operatorname{alg}}\left(G^{\sharp}\right) \rightarrow \pm 1 .
$$

6.3.2. Let $H$ denote the Langlands dual of $G^{\sharp}$, viewed as an algebraic group over $E$. Note that the group

$$
\operatorname{Hom}\left(\pi_{1, \operatorname{alg}}\left(G^{\sharp}\right), E^{\times, \text {tors }}\right)
$$

identifies with $Z_{H}(E)^{\text {tors }}$, where $Z_{H}$ denotes the center of $H$.

Using Corollary 4.3.5, we interpret $\left(\mathcal{G}^{\pi_{1, \mathrm{alg}} \otimes \mathbb{G}_{m}}\right)^{\text {com }}$ as a $Z_{H}(E)^{\text {tors }}$-gerbe, denoted $\mathcal{G}_{Z}$. Furthermore, we interpret the above map $\epsilon$ as a homomorphism

$$
\epsilon: \pm 1 \rightarrow Z_{H}(E)^{\text {tors }}
$$


6.3.3. We will refer to the triple

$$
\left(H, \mathcal{G}_{Z}, \epsilon\right)
$$

as the metaplectic Langlands dual datum corresponding to $\mathcal{G}^{G}$.

6.3.4. Recall the twisting construction of Sect. 1.7.2. Applying it to the constant sheaf of (symmetric monoidal) categories over $X$ with value $\operatorname{Rep}(H)$, i.e.,

$$
\operatorname{Rep}(H) \otimes \operatorname{Shv}_{/ X}
$$

and $\mathcal{A}$ being the constant commutative group-prestack with value $Z_{H}(E)^{\text {tors }}$, i.e.,

$$
Z_{H}(E)^{\text {tors }} \times X
$$

we obtain a new sheaf of (symmetric monoidal) categories over $X$, denoted

$$
\operatorname{Rep}(H)_{\mathcal{S}_{Z}} \text {. }
$$

Let $\operatorname{Rep}(H)_{\mathcal{S}_{Z}}(X)$ denote the (symmetric monoidal) category of its global sections (see Sect. 1.6.8). The category $\operatorname{Rep}(H)_{\mathcal{S}_{Z}}(X)$ carries a naturally defined t-structure.

6.3.5. We now introduce the notion of twisted local system for the metaplectic Langlands dual datum, understood as a triple (6.4). Namely, this is by definition a symmetric monoidal t-exact functor

$$
\operatorname{Rep}(H)_{\mathcal{S}_{Z}}(X) \rightarrow \operatorname{Shv}(X)
$$

In Sect. 8.5 we will formulate a precise relationship between twisted local systems in the above sense and objects appearing in the global metaplectic geometric theory.

Remark 6.3.6. Presumably, twisted local systems as defined above are the same as Galois representations into the metaplectic L-group, as defined in [We].

6.4. Digression: (pre)factorization categories arising from symmetric monoidal categories. In this subsection we will explain a procedure that produces prefactorization categories from symmetric monoidal categories. The source of the metaplectic geometric Satake functor will be a factorization category obtained in this way.

For a more detailed discussion see [Ras2, Sect. 6].

6.4.1. Let $\mathcal{C}$ be a symmetric monoidal DG category.

We define the sheaf of categories Fact(e) on Ran as follows. For an affine test scheme $S$ and an $S$-point of Ran given by $I \subset \operatorname{Maps}(S, X)$, let $\operatorname{Tw}(I)$ be the category whose objects are pairs

$$
I \rightarrow J \rightarrow K
$$

(here $J$ and $K$ are sets (automatically, finite and non-empty)), and whose morphisms are commutative diagrams

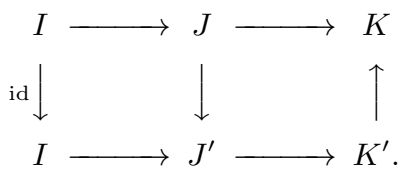

(Note that the arrows between the $K$ 's go in the opposite direction.)

Consider the functor

$$
\mathrm{Tw}(I) \rightarrow \text { DGCat }
$$

that sends an object (6.5) to

$$
\operatorname{Shv}\left(S \underset{X^{I}}{\times} X^{K}\right) \otimes \mathfrak{e}^{\otimes J}
$$

and a morphism (6.6) to

$$
\operatorname{Shv}\left(S \underset{X^{I}}{\times} X^{K}\right) \otimes \mathcal{e}^{\otimes J} \rightarrow \operatorname{Shv}\left(S \underset{X^{I}}{\times} X^{K^{\prime}}\right) \otimes \mathcal{e}^{\otimes J} \rightarrow \operatorname{Shv}\left(\underset{X^{I}}{\times} X^{K^{\prime}}\right) \otimes \mathcal{e}^{\otimes J^{\prime}},
$$


where the first arrow is direct image along

$$
S \underset{X^{I}}{\times} X^{K} \rightarrow S \underset{X^{I}}{\times} X^{K^{\prime}}
$$

and the second arrow is the functor $\mathcal{C}^{\otimes J} \rightarrow \mathcal{C}^{\otimes J^{\prime}}$ given by the symmetric monoidal structure on $\mathcal{C}$.

Finally, we let the value of Fact $(\mathcal{C})$ on $(S, I)$ be the object of DGCat equal to the colimit of the functor (6.7) over $\operatorname{Tw}(I)$.

By construction, Fact(C) admits a prefactorization structure as a sheaf of symmetric monoidal DG categories.

Remark 6.4.2. One can show that Fact(C), regarded as a presheaf on Ran is in fact quasi-coherent, and that its prefactorization structure is actually a factorization structure.

6.4.3. Let Fact $(\mathcal{C})(\operatorname{Ran})$ denote the category of global sections of Fact( $(\mathcal{C})$ over Ran.

As in [Ga5, Sect. 4.2], the (symmetric) monoidal structure on Fact(e) and the operation of union of finite sets makes Fact $(\mathcal{C})(\operatorname{Ran})$ into a non-unital (symmetric) monoidal category.

6.4.4. Let $A$ be a $\Omega$ acting by automorphisms of th $\Omega$ mmetric monoidal structure of $\mathcal{C}$.

Let $\mathcal{G}_{A}$ be an $A$-gerbe over $X$. We can twist the construction of Fact($(\mathcal{C})$ and consider the sheaf on Ran of symmetric monoidal DG categories Fact $(\mathcal{C})_{\mathcal{S}_{A}}$.

6.4.5. Let now $\epsilon$ be a 2 -torsion element of $A$. Using the gerbe $\mathcal{G}^{\epsilon}$ from Sect. 4.5.1, we can further twist $\operatorname{Fact}(\mathcal{C})_{\mathcal{S}_{A}}$ to obtain a (pre)factorization sheaf of monoidal DG categories, denoted Fact $(\mathcal{C})_{\mathcal{S}_{A}}^{\epsilon}$.

Note, however, that by Remark 4.5.5, we have a canonical identification

$$
\operatorname{Fact}(\mathcal{C})_{\mathcal{S}_{A}}(\operatorname{Ran}) \simeq \operatorname{Fact}(\mathcal{C})_{\mathcal{S}_{A}}^{\epsilon}(\operatorname{Ran})
$$

as monoidal categories.

\subsection{The (pre)factorization category of representations.}

6.5.1. We apply the construction in Sect. 6.4 to

$$
\mathcal{C}=\operatorname{Rep}(H), \quad A=Z_{H}(E)^{\text {tors }}, \quad \mathcal{G}_{A}=\mathcal{G}_{Z},
$$

and $\epsilon$ from (6.4).

We obtain the sheaves of monoidal categories over Ran

$$
\operatorname{Fact}(\operatorname{Rep}(H))_{\mathcal{S}_{Z}} \text { and } \operatorname{Fact}(\operatorname{Rep}(H))_{\mathcal{S}_{Z}}^{\epsilon}
$$

and the corresponding monoidal categories

$$
\operatorname{Fact}(\operatorname{Rep}(H))_{\mathcal{S}_{Z}}(\operatorname{Ran}) \simeq \operatorname{Fact}(\operatorname{Rep}(H))_{\mathcal{S}_{Z}}^{\epsilon}(\operatorname{Ran}) .
$$

6.5.2. Example of tori. Let $\mathcal{G}^{T}$ be a multiplicative factorization gerbe on $\mathrm{Gr}_{T}$. In this case,

$$
\operatorname{Shv}_{\mathcal{G}^{T}}\left(\mathrm{Gr}_{T}\right) / \operatorname{Ran}
$$

is naturally a sheaf of monoidal DG categories on Ran, equipped with a (pre)factorization structure.

Note also that by Proposition 4.3.7(a), we have $T^{\sharp}=T$, and so $H \simeq \check{T}$. It is straightforward to show explicitly (see [Re, Proposition IV.5.2]) that we have a canonical isomorphism

$$
\operatorname{Fact}(\operatorname{Rep}(\check{T}))_{\mathcal{G}_{Z}}^{\epsilon} \simeq \operatorname{Shv}_{\mathcal{G}^{T}}\left(\operatorname{Gr}_{T}\right) / \operatorname{Ran}
$$

as sheaves of (pre)factorization monoidal categories.

6.5.3. Unwinding the construction, we obtain that the category of sections of $\operatorname{Fact}(\operatorname{Rep}(H))_{\mathcal{S}_{Z}}$ on $X$ (with respect to the canonical map $X \rightarrow \operatorname{Ran}(X)$ ) is the category

$$
\operatorname{Rep}(H)_{\mathcal{G}_{Z}}(X)
$$

introduced in Sect. 6.3.4. 
6.5.4. Let now $\sigma$ be a twisted local system on $X$ as defined in Sect. 6.3.5. The functoriality of the construction in Sect. 6.4 defines a (symmetric) monoidal functor

$$
\operatorname{Fact}(\operatorname{Rep}(H))_{\mathcal{S}_{Z}}(\operatorname{Ran}) \rightarrow \operatorname{Shv}(\operatorname{Ran})
$$

Assume now that $X$ is complete. Composing with the functor of direct image

$$
\operatorname{Shv}(\operatorname{Ran}) \rightarrow \text { Vect }
$$

we thus obtain a functor

$$
\operatorname{Ev}_{\sigma}: \operatorname{Fact}(\operatorname{Rep}(H))_{\mathcal{S}_{Z}}(\operatorname{Ran}) \rightarrow \operatorname{Vect} .
$$

We will use the functor (6.10) for the definition of the notion of twisted Hecke eigensheaf with respect to $\sigma$.

6.6. The (derived) stack of twisted local systems. In this subsection we will assume that $\operatorname{char}(k)=0$, and that our sheaf-theory is that of D-modules (in particular, the field of coefficients $E$ equals $k$ ).

Assume that $X$ is complete. Starting from the pair $\left(H, \mathcal{G}_{Z}\right)$ appearing in the triple $\left(H, \mathcal{G}_{Z}, \epsilon\right)$ of the metaplectic dual datum, we will construct the derived stack LocSys $\mathcal{G}_{H}$ of $\mathcal{G}_{Z}$-twisted local systems on $X$. Its $k$-points will be the twisted local systems as defined in Sect. 6.3.5.

6.6.1. We will follow the strategy of [AG, Sect. 10.2]. For a derived affine scheme $S$, we set

$$
\operatorname{Maps}\left(S, \operatorname{LocSys}_{H}^{\mathcal{G}_{Z}}\right)
$$

to be the space of right $t$-exact symmetric monoidal functors

$$
\operatorname{Rep}(H)_{\mathcal{G}_{Z}}(X) \rightarrow \mathrm{QCoh}(S) \otimes \operatorname{Shv}(X) .
$$

One shows that $\operatorname{LocSys}_{H}^{\mathcal{S}_{Z}}$ defined in this way is representable by a quasi-smooth derived algebraic stack (see [AG, Sect. 8.1] for what this means).

6.6.2. As in [Ga5, Sect. 4.3], we have a canonically defined (symmetric) monoidal functor

$$
\operatorname{Loc}: \operatorname{Fact}(\operatorname{Rep}(H))_{\mathcal{G}_{Z}}(\operatorname{Ran}) \rightarrow \operatorname{QCoh}\left(\operatorname{LocSys}_{H}^{\mathcal{G}_{Z}}\right)
$$

The following is proved in the same way as [Ga5, Proposition 4.3.4 $]^{14}$ :

Proposition 6.6.3. The functor (6.11) is a localization, i.e., it admits a fully faithful right adjoint.

\section{FACTORIZATION GERBES ON LOOP GROUPS}

In this section we will perform a crucial geometric construction that will explain why our definition of geometric metaplectic datum was "the right thing to do":

We will show that a factorization gerbe on $\mathrm{Gr}_{G}$ give rise to a (factorization) gerbe on (the factorization version of) the loop group of $G$.

\subsection{Digression: factorization loop and arc spaces.}

\footnotetext{
${ }^{14}$ The proof is reproduced in [Ro, Sect. 1.3].
} 
7.1.1. For an affine test scheme $S$ and an $S$-point of Ran, given by a finite set $I \subset \operatorname{Maps}(S, X)$, let $\hat{\mathcal{D}}_{I}$ be the corresponding relative formal disc:

By definition, $\hat{\mathcal{D}}_{I}$ is the formal scheme equal to the completion of $S \times X$ along the union of the graphs of the maps $S \rightarrow X$ corresponding to the elements of $I$.

Note that for a finite set $J$ and a point

$$
\left\{I_{j}, j \in J\right\} \in \operatorname{Ran}_{\mathrm{disj}}^{J}
$$

we have

$$
\hat{\mathcal{D}}_{I} \simeq \bigsqcup_{j} \hat{\mathcal{D}}_{I_{j}}
$$

where $I=\underset{j}{\sqcup} I_{j}$.

7.1.2. Since $S$ was assumed affine, $\hat{\mathcal{D}}_{I}$ is an ind-object in the category $\mathrm{Sch}^{\text {aff }}$. Let $\mathcal{D}_{I}$ be the affine scheme corresponding to the formal scheme $\hat{\mathcal{D}}_{I}$, i.e., the image of $\hat{\mathcal{D}}_{I}$ under the functor

$$
\text { colim }: \operatorname{Ind}\left(\mathrm{Sch}^{\text {aff }}\right) \rightarrow \mathrm{Sch}^{\text {aff }} \text {. }
$$

In other words, if

$$
\hat{\mathcal{D}}_{I} \simeq \operatorname{colim}_{\alpha} Z_{\alpha}
$$

where $Z_{\alpha}=\operatorname{Spec}\left(A_{\alpha}\right)$ and the colimit is taken in PreStk, then $\mathcal{D}_{I}=\operatorname{Spec}(A)$, where

$$
A=\lim _{\alpha} A_{\alpha}
$$

Let $\stackrel{\circ}{\mathcal{D}}_{I}$ be the open subscheme of $\mathcal{D}_{I}$, obtained by removing the closed subscheme equal to the union of the graphs of the maps $S \rightarrow X$ corresponding to the elements of $I$.

7.1.3. Let $Z$ be a prestack. We define the prestacks $\mathfrak{L}^{+}(Z)$ (resp., $\mathfrak{L}(Z)$ ) over Ran as follows.

For an affine test scheme $S$ and an $S$-point of Ran, given by a finite set $I \subset \operatorname{Maps}(S, X)$, its lift to an $S$-point of $\mathfrak{L}^{+}(Z)$ (resp., $\mathfrak{L}(Z)$ ) is the datum of a map $\mathcal{D}_{I} \rightarrow Z$ (resp., $\stackrel{\circ}{\mathcal{D}}_{I} \rightarrow Z$ ).

The isomorphisms (7.1) imply that $\mathfrak{L}^{+}(Z)$ and $\mathfrak{L}(Z)$ are naturally factorzation prestacks over Ran.

7.1.4. Assume for a moment that $Z$ is an affine scheme. Note that in this case the definition of $\mathfrak{L}^{+}(Z)$, the datum of a map $\mathcal{D}_{I} \rightarrow Z$ is equivalent to that of a map of prestacks $\hat{\mathcal{D}}_{I} \rightarrow Z$.

Assume now that $Z$ is a smooth scheme of finite type (but not necessarily affine). Then one shows that for every $S \rightarrow$ Ran, the fiber product

$$
S \underset{\text { Ran }}{\times} \mathfrak{L}^{+}(Z)
$$

is a projective limit (under smooth maps) of smooth affine schemes over $S$.

\subsection{Factorization loop and arc groups.}

7.2.1. Let us recall that the Beauville-Laszlo Theorem says that the definition of $\mathrm{Gr}_{G}$ can be rewritten in terms of the pair $\stackrel{\circ}{\mathcal{D}}_{I} \subset \mathcal{D}_{I}$.

Namely, given $I$ as above, the datum of its lift to a point of $\operatorname{Gr}_{G}$ is a pair $\left(\mathcal{P}_{G}, \alpha\right)$, where $\mathcal{P}_{G}$ is a $G$-bundle on $\mathcal{D}_{I}$, and $\alpha$ is the trivialization of $\left.\mathcal{P}_{G}\right|_{\mathcal{D}_{I}}$. (Note that restriction along $\hat{\mathcal{D}}_{I} \rightarrow \mathcal{D}_{I}$ induces an equivalence between the category of $G$-bundles on $\mathcal{D}_{I}$ and that on $\hat{\mathcal{D}}_{I}$.)

In other words, the Beauville-Laszlo says that restriction along

$$
\left(\stackrel{\circ}{\mathcal{D}}_{I} \subset \mathcal{D}_{I}\right) \rightarrow\left(U_{I} \subset S \times X\right)
$$

induces a bijection on the corresponding pairs $\left(\mathcal{P}_{G}, \alpha\right)$. In the above formula, the notation $U_{I}$ is as in Sect. 2.3.1. 
7.2.2. This interpretation of $\operatorname{Gr}_{G}$ shows that the group-prestack $\mathfrak{L}(G)$ acts naturally on $\operatorname{Gr}_{G}$, with the stabilizer of the unit section being $\mathfrak{L}^{+}(G)$. Furthermore, the natural map

$$
\mathfrak{L}(G) / \mathfrak{L}^{+}(G) \rightarrow \operatorname{Gr}_{G},
$$

is an isomorphism, where the quotient is understood in the sense of stacks in the étale topology.

The isomorphism (7.2) implies that for every $S \rightarrow$ Ran, the fiber product

$$
S \underset{\operatorname{Ran}}{\times} \mathfrak{L}(G),
$$

is an ind-scheme over $S$.

7.2.3. Recall that given a group-prestack $\mathcal{H}$ over a base $Z$, we can talk about a gerbe over $\mathcal{H}$ being multiplicative, i.e., compatible with the group-structure.

In particular, we can consider the spaces

$$
\operatorname{FactGe}_{A}^{\text {mult }}(\mathfrak{L}(G)) \text { and } \operatorname{FactGe}_{A}^{\text {mult }}\left(\mathfrak{L}^{+}(G)\right)
$$

of multiplicative factorization gerbes on $\mathfrak{L}(G)$ and $\mathfrak{L}^{+}(G)$, respectively.

7.2.4. The isomorphism (7.2) defines a map

$$
\operatorname{FactGe}_{A}^{\text {mult }}(\mathfrak{L}(G)) \underset{\operatorname{FactGe}_{A}^{\text {mult }}(\mathfrak{L}+(G))}{\times} * \rightarrow \operatorname{FactGe}_{A}\left(\operatorname{Gr}_{G}\right) .
$$

The following result is established in [Re, Theorem III.2.10]:

Proposition 7.2.5. The map (7.3) is an isomorphism.

We will sketch the proof of this proposition in Sect. 7.5. It consists of explicitly constructing the inverse map.

7.2.6. Let us restate Proposition 7.2.5 in words. It says that, given a factorization gerbe on $\mathrm{Gr}_{G}$, its pullback under the projection

$$
\mathfrak{L}(G) \rightarrow \operatorname{Gr}_{G},
$$

carries a uniquely defined multiplicative structure that is compatible with that of factorization and the trivialization of the further restriction of our gerbe to $\mathfrak{L}^{+}(G)$.

7.3. The $\mathfrak{L}^{+}(G)$-equivariant structure. The main step in constructing the map

$$
\operatorname{FactGe}_{A}\left(\operatorname{Gr}_{G}\right) \rightarrow \operatorname{FactGe}_{A}^{\text {mult }}(\mathfrak{L}(G)) \underset{\operatorname{FactGe}_{A}^{\operatorname{mult}}(\mathfrak{L}+(G))}{\times} *,
$$

inverse to (7.3), consists of constructing a (canonical) structure of equivariance with respect to $\mathfrak{L}^{+}(G)$ on a given factorization gerbe $\mathcal{G}$ on $\mathrm{Gr}_{G}$. We will explain this construction in the present subsection.

7.3.1. For a non-negative integer $n$, let

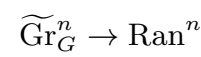

be the $n$-fold convolution diagram. I.e., for an $S$-point of $\operatorname{Ran}^{n}$

$$
\left\{I_{j}, 1 \leq j \leq n\right\} \in \operatorname{Ran}^{n}, \quad I_{j} \subset \operatorname{Hom}(S, X),
$$

its lift to an $S$-point of $\widetilde{\mathrm{Gr}_{G}^{n}}$ consists of a string of $G$-bundles

$$
\mathcal{P}_{G}^{1}, \mathcal{P}_{G}^{2}, \ldots, \mathcal{P}_{G}^{n}
$$

on $S \times X$, together with identifications

$$
\left.\left.\mathcal{P}_{G}^{0}\right|_{U_{I_{1}}} \stackrel{\alpha_{1}}{\simeq} \mathcal{P}_{G}^{1}\right|_{U_{I_{1}}},\left.\left.\mathcal{P}_{G}^{1}\right|_{U_{I_{2}}} \stackrel{\alpha_{2}}{\simeq} \mathcal{P}_{G}^{2}\right|_{U_{I_{2}}}, \ldots,\left.\left.\mathcal{P}_{G}^{n-1}\right|_{U_{I_{n}}} \stackrel{\alpha_{n}}{\simeq} \mathcal{P}_{G}^{n}\right|_{U_{I_{n}}},
$$

where $\mathcal{P}_{G}^{0}$ denotes the trivial $G$-bundle.

We have a naturally defined map

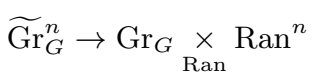


that sends the above data to

$$
\left(I:=\sqcup_{j} I_{j}, \mathcal{P}_{G}=\mathcal{P}_{G}^{n}, \alpha=\alpha_{n} \circ \ldots \circ \alpha_{1}\right) .
$$

This map is an isomorphism over $\operatorname{Ran}_{\mathrm{disj}}^{n}$.

7.3.2. In Sects. 7.3.3-7.3.5 below we will explain that for a decomposition $n=n_{1}+n_{2}$, we can view $\widetilde{\mathrm{Gr}}_{G}^{n}$ as a twisted product

$$
\widetilde{\mathrm{Gr}}_{G}^{n_{1}+n_{2}} \simeq \widetilde{\mathrm{Gr}_{G}^{n_{1}}} \widetilde{\times \mathrm{Gr}_{G}^{n_{2}}}
$$

which identifies with the usual product $\widetilde{\mathrm{Gr}}_{G}^{n_{1}} \times \widetilde{\mathrm{Gr}}_{G}^{n_{2}}$ when restricted to $(\operatorname{Ran} \times \operatorname{Ran})_{\text {disj }}$, where

$$
\operatorname{Ran}^{n} \simeq \operatorname{Ran}^{n_{1}} \times \operatorname{Ran}^{n_{2}} \rightarrow \operatorname{Ran} \times \operatorname{Ran}
$$

corresponds to the projection $\operatorname{Ran}^{n_{1}} \rightarrow$ Ran on the last coordinate and the projection $\operatorname{Ran}^{n_{2}} \rightarrow \operatorname{Ran}$ on the first component.

This is a well-known construction and the reader familiar with it can safely skip it.

7.3.3. Denote $I:=\sqcup_{j} I_{j}$. Using the Beauville-Laszlo theorem, we can rewrite the data involved in $\widetilde{\operatorname{Gr}}_{G}^{n}$ by letting our $G$-bundles (7.5) be defined on $\mathcal{D}_{I}$, and $\alpha_{j}$ be defined on the open subscheme obtained by removing the closed subscheme equal to the union of the graphs of the maps $S \rightarrow X$ corresponding to the elements of $I_{j}$.

This description shows that $\widetilde{\mathrm{Gr}}_{G}^{n}$ carries an action of the group-prestack

$$
\mathfrak{L}(G) \underset{\operatorname{Ran}}{\times} \operatorname{Ran}^{n},
$$

where the map $\operatorname{Ran}^{n} \rightarrow$ Ran is the projection on the first component.

7.3.4. For $n=n_{1}+n_{2}$, consider the prestack over Ran $\times$ Ran equal to $\operatorname{Ran} \times \widetilde{\mathrm{Gr}_{G}} \tilde{n}_{2}$; where $\widetilde{\mathrm{Gr}_{G}^{n_{2}}} \rightarrow \operatorname{Ran}$ is the projection on the first component. The above prestack is equipped with an action of the groupprestack $\operatorname{Ran} \times \mathfrak{L}(G)$, and in particular $\operatorname{Ran} \times \mathfrak{L}^{+}(G)$.

Consider the prestack $\widetilde{\mathrm{Gr}_{G}^{n_{1}}} \times$ Ran over Ran $\times$ Ran, where $\widetilde{\mathrm{Gr}_{G}^{n}} \tilde{n}_{1} \rightarrow$ Ran is the projection on the last component. Consider the $\operatorname{Ran} \times \mathfrak{L}^{+}(G)$-torsor, denoted $\mathcal{R}^{n_{1}, n_{2}}$, over $\widetilde{\mathrm{Gr}}_{G}^{n_{1}} \times \operatorname{Ran}$, that sends

$$
\left(I_{1}, \ldots, I_{n_{1}}, \mathcal{P}_{G}^{1}, \mathcal{P}_{G}^{2}, \ldots, \mathcal{P}_{G}^{n_{1}}, \alpha_{1}, \ldots, \alpha_{n_{1}}, I^{\prime}\right)
$$

to the set of trivializations of the $G$-bundle $\mathcal{P}_{G}^{n_{1}}$ over $\mathcal{D}_{I^{\prime}}$.

Note the above torsor is canonically trivialized over $(\operatorname{Ran} \times \operatorname{Ran})_{\text {disj }}$.

7.3.5. Consider the projection

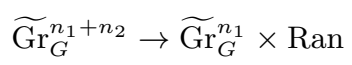

that sends

$$
\left(I_{1}, \ldots, I_{n_{1}+n_{2}}, \mathcal{P}_{G}^{1}, \mathcal{P}_{G}^{2}, \ldots, \mathcal{P}_{G}^{n_{1}+n_{2}}, \alpha_{1}, \ldots, \alpha_{n_{1}+n_{2}}\right) \mapsto\left(I_{1}, \ldots, I_{n_{1}}, \mathcal{P}_{G}^{1}, \mathcal{P}_{G}^{2}, \ldots, \mathcal{P}_{G}^{n_{1}}, \alpha_{1}, \ldots, \alpha_{n_{1}}, I_{n_{2}}\right) .
$$

We observe that $\widetilde{\mathrm{Gr}_{G}} n_{1}+n_{2}$, viewed as a prestack over $\widetilde{\mathrm{Gr}_{G}}{ }_{G} \times \operatorname{Ran}$, is obtained by twisting $\operatorname{Ran} \times \widetilde{\mathrm{Gr}}_{G}^{n_{2}}$, viewed as a prestack over $\operatorname{Ran} \times \operatorname{Ran}$, equipped with an action of $\operatorname{Ran} \times \mathfrak{L}^{+}(G)$, by means of the $\operatorname{Ran} \times \mathfrak{L}^{+}(G)$-torsor $\mathcal{R}^{n_{1}, n_{2}}$.

In other words, we can think of $\widetilde{G r}_{G}^{n_{1}+n_{2}}$ as a twisted product

$$
\widetilde{\mathrm{Gr}}_{G}^{n_{1}+n_{2}} \simeq \widetilde{\mathrm{Gr}_{G}^{n_{1}}} \widetilde{\times \widetilde{G r}_{G}^{n_{2}}},
$$

where we use the $\operatorname{Ran} \times \mathfrak{L}^{+}(G)$-torsor $\mathcal{R}^{n_{1}, n_{2}}$ over $\widetilde{\mathrm{Gr}_{G}}{ }_{G}^{n_{1}} \times \operatorname{Ran}$ and the $\operatorname{Ran} \times \mathfrak{L}^{+}(G)$-action on $\operatorname{Ran} \times \widetilde{\mathrm{Gr}}_{G}^{n_{2}}$ to form the twisted product. 
Note that we have a commutative diagram (7.8)

$$
\begin{aligned}
& \widetilde{\mathrm{Gr}_{G}^{n_{1}}+n_{2}} \underset{\operatorname{Ran}^{n_{1}+n_{2}}}{\times}\left(\operatorname{Ran}^{n_{1}+n_{2}}\right)_{\text {disj }} \stackrel{(7.7)}{\sim} \quad\left(\widetilde{\mathrm{Gr}}_{G}^{n_{1}} \widetilde{\times} \widetilde{\mathrm{Gr}_{G}^{n_{2}}}\right)_{\operatorname{Ran}^{n_{1}+n_{2}}}^{\times}\left(\operatorname{Ran}^{n_{1}+n_{2}}\right)_{\text {disj }} \\
& (7.6) \downarrow \sim \quad \sim \downarrow \text { trivialization of } \mathcal{R}^{n_{1}, n_{2}} \text { on }(\operatorname{Ran} \times \operatorname{Ran})_{\mathrm{disj}} \\
& \operatorname{Gr}_{G} \underset{\operatorname{Ran}}{\times}\left(\operatorname{Ran}^{n_{1}+n_{2}}\right)_{\text {disj }} \quad\left(\widetilde{\operatorname{Gr}}_{G}^{n_{1}} \times{\widetilde{\operatorname{Gr}_{G}}}_{n_{2}}^{n_{2}} \underset{\operatorname{Ran}^{n_{1}+n_{2}}}{\times}\left(\operatorname{Ran}^{n_{1}+n_{2}}\right)_{\text {disj }}\right. \\
& \text { factorization } \downarrow \text { (7.6) } \downarrow \sim \\
& \left(\operatorname{Gr}_{G}\right)^{\times n_{1}+n_{2}} \underset{\operatorname{Ran}^{n_{1}+n_{2}}}{\times}\left(\operatorname{Ran}^{n_{1}+n_{2}}\right)_{\text {disj }} \longrightarrow\left(\left(\operatorname{Gr}_{G}\right)^{\times n_{1}} \times\left(\operatorname{Gr}_{G}\right)^{\times n_{2}}\right)_{\operatorname{Ran}^{n_{1}+n_{2}}}\left(\operatorname{Ran}^{n_{1}+n_{2}}\right)_{\text {disj }}
\end{aligned}
$$

7.3.6. The key observation (proved by reduction to the Cartan subgroup) is that a factorization gerbe $\mathcal{G}$ on $\operatorname{Gr}_{G}$ admits a unique structure of equivariance with respect to $\mathfrak{L}^{+}(G)$ that has the following property:

In the setting of Sects. 7.3.4-7.3.5 take $n_{1}=n_{2}=1$, and consider the twisted product $\mathcal{G} \widetilde{\otimes} \mathcal{G}$, which is a well-defined gerbe on $\widetilde{\mathrm{Gr}}_{G}^{2}$ due to the identification of (7.7) and the chosen structure of equivariance with respect to $\mathfrak{L}^{+}(G)$ on $\mathcal{G}$.

We require that $\mathcal{G} \widetilde{\otimes} \mathcal{G}$ should admit an identification with the pullback of $\mathcal{G}$ under the map

$$
{\widetilde{\mathrm{Gr}_{G}}}_{G}^{2} \stackrel{(7.6)}{\longrightarrow} \operatorname{Gr}_{G} \underset{\operatorname{Ran}}{\times}(\operatorname{Ran} \times \operatorname{Ran}) \rightarrow \mathrm{Gr}_{G}
$$

which extends the already existing identification over

$$
\widetilde{\mathrm{Gr}}_{G}^{2} \underset{\operatorname{Ran} \times \operatorname{Ran}}{\times}(\operatorname{Ran} \times \operatorname{Ran})_{\mathrm{disj}},
$$

given by the factorization structure on $\mathcal{G}$ via the diagram $(7.8)$ for $n_{1}=n_{2}=1$ :

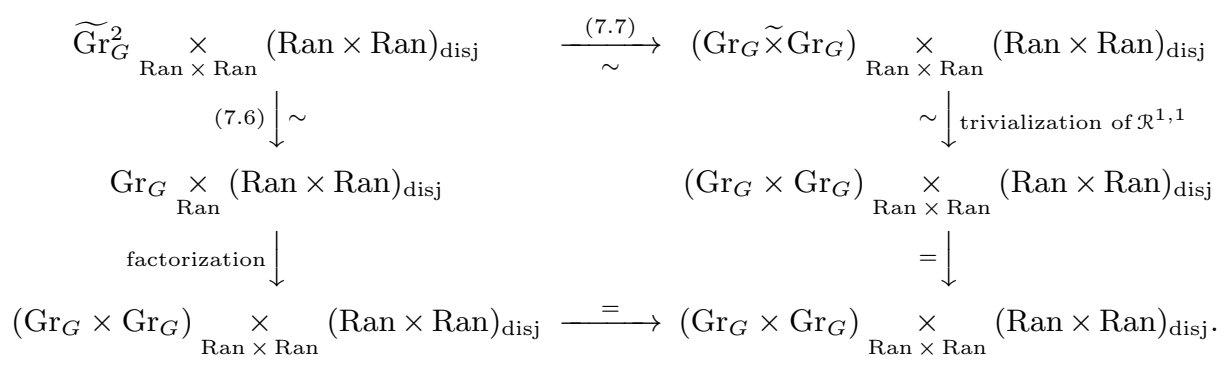

7.4. Another view on the bilinear form. The $\mathfrak{L}^{+}(G)$-equivariant structure on $\mathcal{G}$ gives rise to the following interpretation of the bilinear form attached to $\mathcal{G}$, when $G$ is a torus $T$.

7.4.1. Namely, choose an arbitrary point $x \in X$, and consider the restrictions

$$
\operatorname{Gr}_{G, x}:=\{x\} \underset{\operatorname{Ran}}{\times} \operatorname{Gr}_{G} \text { and } \mathfrak{L}^{+}(G)_{x}:=\{x\} \underset{\operatorname{Ran}}{\times \mathfrak{L}^{+}}(G) \simeq G\left(\hat{\mathcal{O}}_{x}\right) .
$$

We obtain that the $A$-gerbe restriction $\left.\mathcal{G}\right|_{\operatorname{Gr}_{G, x}}$ is equivariant with respect to $G\left(\hat{\mathcal{O}}_{x}\right)$.

7.4.2. For $G=T$, since $T$ is commutative, the action of $T\left(\hat{\mathcal{O}}_{x}\right)$ on $\operatorname{Gr}_{T, x}$ is trivial. Hence, for every $\lambda \in \Lambda$, the action of $T\left(\hat{\mathcal{O}}_{x}\right)$ on the corresponding point of $\operatorname{Gr}_{T, x}$ defines a multiplicative $A$-torsor on $T\left(\hat{\mathcal{O}}_{x}\right)$.

Since the elements of $A$ have orders prime to $\operatorname{char}(k)$, the above multiplicative $A$-torsor is pulled back from $T$, and by Kummer theory, the latter is given by a homomorphism

$$
\Lambda \rightarrow A(-1) .
$$


Thus, we have constructed a map

$$
\Lambda \rightarrow \operatorname{Hom}(\Lambda, A(-1))
$$

7.4.3. By unwinding the constructions, one shows that (7.9) equals one coming from the bilinear form attached to $\mathcal{G}$ and our chosen element $\lambda \in \Lambda$.

\subsection{Construction of the inverse map in Proposition 7.2.5.}

7.5.1. For a non-negative integer $n$, consider the prestack

$$
Z^{n}:=\mathfrak{L}^{+}(G) \backslash\left(\widetilde{\mathrm{Gr}}^{n} \underset{\operatorname{Ran}^{n}}{\times} \operatorname{Ran}\right),
$$

where $\operatorname{Ran} \rightarrow \operatorname{Ran}^{n}$ is the diagonal map.

It is easy to see that as $n$ varies, the prestacks (7.10) form a simplicial object in PreStk/Ran; denote it by $Z^{\bullet}$. Consider its geometric realization $\left|Z^{\bullet}\right|$, viewed as a prestack over Ran, equipped with a factorization structure.

By the construction in Sect. 7.3, a factorization $A$-gerbe on $\operatorname{Gr}_{G}$ gives rise to a 2 -gerbe on $\left|Z^{\bullet}\right|$ with respect to $A$, i.e., a map

equipped with a trivialization of its restriction to

$$
\left|Z^{\bullet}\right| \rightarrow B_{\text {et }}^{3}(A),
$$

$$
B_{\text {et }}\left(\mathfrak{L}^{+}(G)\right)=Z^{0} \rightarrow\left|Z^{\bullet}\right| .
$$

Moreover, the above 2-gerbe is naturally equipped with the factorization structure.

7.5.2. Note now that we have the (simplicial) identification between (7.10) and the Čech nerve of the map

$$
B_{\text {et }}\left(\mathfrak{L}^{+}(G)\right) \rightarrow B_{\text {et }}(\mathfrak{L}(G))
$$

Thus, we obtain a 2-gerbe on $B_{\text {et }}(\mathfrak{L}(G))$, equipped with a trivialization of its restriction to $B_{\text {et }}\left(\mathfrak{L}^{+}(G)\right)$, and equipped with a factorization structure.

The latter datum is equivalent to that of a multiplicative gerbe on $\mathfrak{L}(G)$, equipped with a (multiplicative) trivialization of its restriction to $\mathfrak{L}^{+}(G)$.

\section{Metaplectic geometric Satake}

In section we take $G$ to be reductive. We will define the metaplectic geometric Satake functor and formulate the "metaplectic vanishing conjecture" about the global Hecke action.

We continue to assume that $\operatorname{char}(k) \neq 2$.

8.1. The metaplectic spherical Hecke category. In this subsection we introduce the metaplectic spherical Hecke category, which is the recipient of the metaplectic geometric Satake functor.

8.1.1. Let $\mathcal{G}^{G}$ be a factorization $E^{\times \text {,tors }}$-gerbe on $\operatorname{Gr}_{G}$. We define the sheaf of categories $\left(\operatorname{Sph}_{\mathcal{G} G}\right) / \operatorname{Ran}$ as follows. For an affine test scheme $S$ and an $S$-point of Ran, we define the corresponding category by

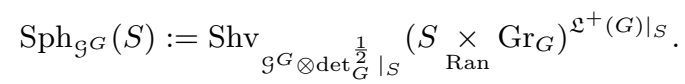

In the above formula, $\left.\mathfrak{L}^{+}(G)\right|_{S}$ denotes the value on $S$ of the factorization group-scheme $\mathfrak{L}^{+}(G)$. The superscript $\left.\mathfrak{L}^{+}(G)\right|_{S}$ indicates the equivariant category with respect to that group-scheme ${ }^{15}$. Note that the latter makes sense due to the equivariance structure on the gerbe $\left.\mathcal{G}^{G} \otimes \operatorname{det}_{G}^{\frac{1}{2}}\right|_{S}$ with respect to $\left.\mathfrak{L}^{+}(G)\right|_{S}$ that was constructed in Sect. 7.3.

By Proposition 7.2.5, we obtain that the operation of convolution product defines on $\left(\mathrm{Sph}_{\mathcal{G} G}\right) / \operatorname{Ran}$ a structure of sheaf of monoidal categories over Ran.

By construction, $\left(\mathrm{Sph}_{\mathcal{G} G}\right) /$ Ran carries a natural prefactorization structure, see Sect. 2.2.3.

\footnotetext{
${ }^{15}$ For a prestack $y$, a group-object $\mathcal{H}$ in PreStk $/ y$ and $Z \in$ PreStk $/ y$, equipped with an action of $\mathcal{H}$, the corresponding equivariant category of sheaves is defined by $\operatorname{Shv}(Z)^{\mathcal{H}}:=\operatorname{Shv}(\mathcal{H} \backslash Z)$.
} 
Remark 8.1.2. One can show that $\left(\mathrm{Sph}_{\mathcal{G} G}\right) / \mathrm{Ran}$, regarded as a sheaf of categories on Ran is in fact quasi-coherent (see Sect. 1.6.5 for what this means), and that the above prefactorization structure is actually a factorization structure.

8.1.3. Let $P$ be a parabolic subgroup of $G$ with Levi quotient $M$. Let us denote by $\mathcal{G}^{M}$ the factorization gerbe on $\mathrm{Gr}_{M}$ corresponding to $\mathcal{G}^{G}$.

The functor (5.5) naturally upgrades to a functor between sheaves of categories

$$
J_{M}^{G}:\left(\mathrm{Sph}_{\mathcal{G} G}\right) / \operatorname{Ran} \rightarrow\left(\mathrm{Sph}_{\mathcal{G} M}\right) / \operatorname{Ran} .
$$

By construction, (8.2) respects the factorization structure, i.e., it is a functor between factorization sheaves of categories.

Remark 8.1.4. We note that the functor (8.2) is not at all compatible with the monoidal structures!

8.2. The metaplectic geometric Satake functor. Metaplectic geometric Satake is a canonically defined functor between (pre)factorization sheaves of monoidal DG categories

$$
\text { Sat : } \operatorname{Fact}(\operatorname{Rep}(H))_{\mathcal{G}_{Z}} \rightarrow\left(\operatorname{Sph}_{\mathcal{G} G}\right) / \operatorname{Ran} .
$$

We will now explain how to obtain this functor from [Re, Theorem IV.8.3 $]^{16}$.

8.2.1. By Sect. 2.1.2, the datum of a functor (8.3) amounts to a compatible collection of functors

$$
\operatorname{Sat}(I): \operatorname{Fact}(\operatorname{Rep}(H))_{\mathcal{G}_{Z}}^{\epsilon}\left(X^{I}\right) \rightarrow\left(\operatorname{Sph}_{\mathcal{G} G}\right) / \operatorname{Ran}\left(X^{I}\right),
$$

where $I$ runs over the category of finite non-empty sets and surjective morphisms.

Both sides in (8.4) are equipped with t-structures; moreover one shows that Fact $(\operatorname{Rep}(H))_{\mathcal{G}_{Z}}^{\epsilon}\left(X^{I}\right)$ identifies with the derived category of its t-structure ${ }^{17}$, i.e., the canonical map of [Lu2, Theorem 1.3.3.2]

$$
D\left(\left(\operatorname{Fact}(\operatorname{Rep}(H))_{\mathcal{S}_{Z}}^{\epsilon}\left(X^{I}\right)\right)^{\bigodot}\right) \rightarrow \operatorname{Fact}(\operatorname{Rep}(H))_{\mathcal{S}_{Z}}^{\epsilon}\left(X^{I}\right)
$$

is an equivalence.

Now, [Re, Theorem IV.8.3] constructs an equivalence of abelian categories

$$
\left(\operatorname{Fact}(\operatorname{Rep}(H))_{\mathcal{G}_{Z}}^{\epsilon}\left(X^{I}\right)\right)^{\odot} \rightarrow\left(\left(\operatorname{Sph}_{\mathcal{G} G}\right) / \operatorname{Ran}\left(X^{I}\right)\right)^{\odot}
$$

Applying [Lu2, Theorem 1.3.3.2] again, we obtain a canonically defined functor

$$
D\left(\left(\operatorname{Fact}(\operatorname{Rep}(H))_{\mathcal{G}_{Z}}^{\epsilon}\left(X^{I}\right)\right)^{\complement}\right) \rightarrow\left(\operatorname{Sph}_{\mathcal{G} G}\right) / \operatorname{Ran}\left(X^{I}\right)
$$

thus giving rise to the desired functor (8.4).

The functoriality with respect to the finite sets $I$, as well as compatibility with factorization is built into the construction.

8.3. Example: metaplectic geometric Satake for tori. In this subsection we let $G=T$ be a torus.

\footnotetext{
${ }^{16}$ For a more detailed discussion on how to carry out this extension see [Ras2, Sect. 6], where the classical (i.e., non-metaplectic situation) is considered, but for this step, there is no difference between the two cases.

${ }^{17}$ Here, the derived category is understood as a DG category, see [Lu2, Sect. 1.3.2].
} 
8.3.1. Let $\Lambda^{\sharp} \subset \Lambda$ denote the kernel of $b$.

Direct image along the inclusion

$$
\mathrm{Gr}_{T^{\sharp}} \rightarrow \mathrm{Gr}_{T}
$$

is a fully faithful functor

$$
\operatorname{Shv}_{\mathcal{G}^{\sharp}}\left(\mathrm{Gr}_{T^{\sharp}}\right) / \operatorname{Ran} \rightarrow \operatorname{Shv}_{\mathcal{G}^{T}}\left(\mathrm{Gr}_{T}\right) / \operatorname{Ran},
$$

where we denote by $\mathcal{G}^{T^{\sharp}}$ the restriction of $\mathcal{G}^{T}$ along (8.6).

In this case, it follows from Sect. 7.4 that the forgetful functor

$$
\left(\operatorname{Sph}_{\mathcal{G}^{T}}\right)_{/ \operatorname{Ran}} \rightarrow \operatorname{Shv}_{\mathcal{G}^{T}}\left(\mathrm{Gr}_{T}\right) / \operatorname{Ran}
$$

factors through the essential image of (8.7), thereby giving rise to a functor

$$
\left(\mathrm{Sph}_{\mathcal{G}^{T}}\right)_{/ \operatorname{Ran}} \rightarrow \mathrm{Shv}_{\mathcal{G}^{\sharp}}\left(\mathrm{Gr}_{T^{\sharp}}\right) / \operatorname{Ran},
$$

compatible with the factorization structures.

8.3.2. Furthermore, since the action of $\mathfrak{L}^{+}(T)$ on $\mathrm{Gr}_{T}$ is trivial, the functor (8.8) admits a canonically defined right inverse

$$
\operatorname{Shv}_{\mathcal{G}^{T^{\sharp}}}\left(\mathrm{Gr}_{T^{\sharp}}\right) / \operatorname{Ran} \rightarrow\left(\operatorname{Sph}_{\mathcal{G}^{T}}\right) / \operatorname{Ran},
$$

which is monoidal and compatible with the factorization structures.

8.3.3. By Proposition 4.3.7(b), the factorization gerbe $\mathcal{G}^{T^{\sharp}}$ carries a canonical multiplicative structure. Recall the equivalence

$$
\operatorname{Fact}(\operatorname{Rep}(H))_{\mathcal{G}_{Z}} \simeq \operatorname{Shv}_{\mathcal{G}^{T \sharp}}\left(\operatorname{Gr}_{T^{\sharp}}\right) / \operatorname{Ran}
$$

of $(6.9)$.

The geometric Satake functor for $T$ is the composite of (8.10) and (8.9).

\subsection{Compatibility with Jacquet functors.}

8.4.1. A key feature of the assignment

$$
\mathcal{G}^{G} \rightsquigarrow \mathcal{G}^{\pi_{1, \mathrm{alg}} \otimes \mathbb{G}_{m}}
$$

of Sect. 6.2.1 is compatibility with parabolics in the following sense.

Note that for a parabolic $P$ of $G$ with Levi quotient $M$, the corresponding reductive group $M^{\sharp}$ identifies with the Levi subgroup of $G^{\sharp}$, attached to the same subset of the Dynkin diagram.

We have a canonical surjection

$$
\pi_{1, \operatorname{alg}}\left(M^{\sharp}\right) \rightarrow \pi_{1, \operatorname{alg}}\left(G^{\sharp}\right),
$$

and the corresponding map of factorization Grassmannians

$$
\mathrm{Gr}_{\pi_{1, \mathrm{alg}}\left(M^{\sharp}\right) \otimes \mathbb{G}_{m}} \rightarrow \mathrm{Gr}_{\pi_{1, \mathrm{alg}}\left(G^{\sharp}\right) \otimes \mathbb{G}_{m}} .
$$

Let $\mathcal{G}^{M}$ be the factorization gerbe on $\operatorname{Gr}_{M}$ that corresponds to $\mathcal{G}^{G}$ under the map of Sect. 5.1.4. Then the multiplicative gerbe $\mathcal{G}^{\pi_{1, \text { alg }}\left(M^{\sharp}\right) \otimes \mathbb{G}_{m}}$ on $\operatorname{Gr}_{\pi_{1, \text { alg }}\left(M^{\sharp}\right) \otimes \mathbb{G}_{m}}$ attached to $\mathcal{G}^{M}$ by Sect. 6.2.1 identifies with the pullback with respect to (8.12) of the multiplicative gerbe $\mathcal{G}^{\pi_{1, \mathrm{alg}}\left(G^{\sharp}\right) \otimes \mathbb{G}_{m}}$ on $\operatorname{Gr}_{\pi_{1, \text { alg }}\left(G^{\sharp}\right) \otimes \mathbb{G}_{m}}$ attached to $\mathcal{G}^{G}$. 
8.4.2. Let $M_{H}$ be the standard Levi quotient in $H$ corresponding to standard Levi $M^{\sharp}$ of $G^{\sharp}$. Corresponding to (8.11) we have the inclusion

$$
Z_{H} \rightarrow Z_{M_{H}}
$$

By the above, this inclusion is compatible with the corresponding datum of

$$
\epsilon: \pm 1 \rightarrow Z_{H}(E)^{\text {tors }} \text { and } \epsilon: \pm 1 \rightarrow Z_{M_{H}}(E)^{\text {tors }}
$$

and the corresponding $Z_{H}(E)^{\text {tors }}$ - and $Z_{M_{H}}(E)^{\text {tors }}$-gerbes on $X$ (we denote both by $\mathcal{G}_{Z}$ ).

Therefore, restriction along $M_{H} \rightarrow H$ defines a monoidal functor

$$
\operatorname{Res}_{M}^{G}: \operatorname{Fact}(\operatorname{Rep}(H))_{\mathcal{S}_{Z}}^{\epsilon} \rightarrow \operatorname{Fact}\left(\operatorname{Rep}\left(M_{H}\right)\right)_{\mathcal{S}_{Z}}^{\epsilon}
$$

compatible with the factorization structures.

8.4.3. The key feature of the monoidal functor (8.3) is that it makes the following diagram commute:

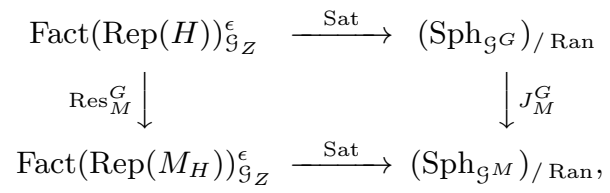

where $J_{M}^{G}$ is the Jacquet functor of (8.2).

8.5. Global Hecke action. In this subsection we will assume that $X$ is complete. We will define the notion of Hecke eigensheaf on $\operatorname{Bun}_{G}$ with respect to a given twisted local system.

8.5.1. Consider category of global sections of $\left(\mathrm{Sph}_{\mathcal{G} G}\right) / \operatorname{Ran}$ over Ran (see Sect. 1.6.8), denote it by

$$
\operatorname{Sph}_{\mathcal{G} G}(\operatorname{Ran}),
$$

and note that it identifies with

$$
\operatorname{Shv}_{\mathcal{G}^{G} \otimes \operatorname{det}_{G}^{\frac{1}{2}}}\left(\mathrm{Gr}_{G}\right)^{\mathfrak{L}^{+}(G)}
$$

As in [Ga5, Sect. 4.4], the monoidal structure on $\left(\mathrm{Sph}_{\mathcal{G} G}\right) / \mathrm{Ran}$, and the operation of union of finite sets, define a (non-unital) monoidal structure on $\operatorname{Sph}_{\mathcal{G} G}$ (Ran).

Moreover, the Hecke action defines a monoidal action of $\operatorname{Sph}_{\mathcal{G}^{G}}(\operatorname{Ran})$ on $\operatorname{Shv}_{\mathcal{G}^{G} \otimes \operatorname{det}_{G}^{\frac{1}{2}}}\left(\operatorname{Bun}_{G}\right)$, where by a slight abuse of notation we denote by the same symbols $\mathcal{G}^{G}$ and $\operatorname{det}_{G}^{\frac{1}{2}}$ the corresponding $E^{\times \text {,tors }}$ gerbes on $\operatorname{Bun}_{G}$, see Sect. 2.3.4.

8.5.2. Passing to global sections over Ran in (8.3), we obtain a monoidal functor

$$
\operatorname{Fact}(\operatorname{Rep}(H))_{\mathcal{G}_{Z}}^{\epsilon}(\operatorname{Ran}) \rightarrow \operatorname{Sph}_{\mathcal{G} G}(\operatorname{Ran})
$$

where we remind that $\operatorname{Fact}(\operatorname{Rep}(H))_{\mathcal{G}_{Z}}^{\epsilon}(\operatorname{Ran})$ denotes the monoidal category of global sections of $\operatorname{Fact}(\operatorname{Rep}(H))_{\mathcal{G}_{Z}}$.

Thus, we obtain a monoidal action of $\operatorname{Fact}(\operatorname{Rep}(H))_{\mathcal{G}_{Z}}^{\operatorname{Ran}}\left(\operatorname{Ran} \operatorname{Shv}_{{ }^{G}{ }^{G} \otimes \operatorname{det}_{G}^{\frac{1}{2}}}\left(\operatorname{Bun}_{G}\right)\right.$. 
8.5.3. Hecke eigensheaves. Let $\sigma$ be a twisted local system on $X$, as defined in Sect. 6.3.5. Recall (see Sect. 6.5.4) that $\sigma$ gives rise to a (symmetric) monoidal functor

$$
\operatorname{Ev}_{\sigma}: \operatorname{Fact}(\operatorname{Rep}(H))_{\mathcal{S}_{Z}}(\operatorname{Ran}) \rightarrow \operatorname{Vect},
$$

and hence, via the monoidal equivalence (6.8) to a monoidal functor

$$
\operatorname{Fact}(\operatorname{Rep}(H))_{\mathcal{G}_{Z}}^{\epsilon}(\operatorname{Ran}) \rightarrow \operatorname{Vect},
$$

which we will denote by the same symbol $\mathrm{Ev}_{\sigma}$.

We define the category of twisted Hecke eigensheaves with respect to $\sigma$ to be the DG category of functors of $\operatorname{Fact}(\operatorname{Rep}(H))_{\mathcal{G}_{Z}}^{\epsilon}(\operatorname{Ran})$-module categories

$$
\text { Vect } \rightarrow \operatorname{Shv}_{\mathcal{G}^{G} \otimes \operatorname{det}_{G}^{\frac{1}{2}}}\left(\operatorname{Bun}_{G}\right),
$$

where $\operatorname{Fact}(\operatorname{Rep}(H))_{\mathcal{G}_{Z}}^{\epsilon}(\operatorname{Ran})$ acts on Vect via $\operatorname{Ev}_{\sigma}$ and on $\operatorname{Shv}_{\mathcal{G}^{G} \otimes \operatorname{det}_{G}^{\frac{1}{2}}}\left(\operatorname{Bun}_{G}\right)$ as in Sect. 8.5.2.

8.6. The metaplectic vanishing conjecture. We continue to assume that $X$ is complete. In this subsection we will assume that $k$ has characteristic 0 , and that our sheaf theory is that of D-modules. Recall (see Sect. 6.6) that in this case we have the (derived) stack $\operatorname{LocSys}_{H}^{\mathcal{G}_{Z}}$.

We will state a conjecture to the effect that the (non-unital) monoidal category

$$
\mathrm{QCoh}\left(\operatorname{LocSys}_{H}^{\mathcal{G}_{Z}}\right)
$$

acts on the category

$$
\operatorname{Shv}_{\mathcal{G}^{G} \otimes \operatorname{det}_{G}^{\frac{1}{2}}}\left(\operatorname{Bun}_{G}\right)
$$

8.6.1. Recall (see Proposition 6.6.3) that we have a (symmetric) monoidal functor

$$
\operatorname{Loc}: \operatorname{Fact}(\operatorname{Rep}(H))_{\mathcal{G}_{Z}}(\operatorname{Ran}) \rightarrow \operatorname{QCoh}\left(\operatorname{LocSys}_{H}^{\mathfrak{S}_{Z}}\right)
$$

of (6.11) with a fully faithful right adjoint. Hence, by (6.8), we obtain a monoidal functor, denoted by the same symbol

$$
\operatorname{Loc}: \operatorname{Fact}(\operatorname{Rep}(H))_{\mathcal{G}_{Z}}^{\epsilon}(\operatorname{Ran}) \rightarrow \operatorname{QCoh}\left(\operatorname{LocSys}_{H}^{\mathcal{G}_{Z}}\right)
$$

also with a fully faithful right adjoint.

The following is an analog of [Ga5, Theorem 4.5.2] in the metaplectic case:

Conjecture 8.6.2. If an object of $\operatorname{Fact}(\operatorname{Rep}(H))_{\mathcal{G}_{Z}}^{\epsilon}(\operatorname{Ran})$ lies in the kernel of the functor Loc, then this object acts by zero on $\mathrm{Shv}_{\mathcal{G}^{G} \otimes \operatorname{det}_{G}^{\frac{1}{2}}}\left(\operatorname{Bun}_{G}\right)$.

This conjecture can be restated as follows:

Conjecture 8.6.3. The action of $\operatorname{Fact}(\operatorname{Rep}(H))_{\mathcal{S}_{Z}}^{\epsilon}(\operatorname{Ran})$ on $\mathrm{Shv}_{{ }_{\mathcal{G}^{G}} \otimes \operatorname{det}_{G}^{\frac{1}{2}}}\left(\operatorname{Bun}_{G}\right)$ (uniquely) factors through an action of QCoh $\left(\operatorname{LocSys}_{H}^{\mathcal{G}}\right)$.

Remark 8.6.4. Using Fourier-Mukai transform, one can show that Conjecture 8.6.2 holds when $G=T$ is a torus. 
8.6.5. Let us assume Conjecture 8.6.3, so that $\mathrm{Shv}_{\mathcal{G}^{G} \otimes \operatorname{det}_{G}^{\frac{1}{2}}}\left(\operatorname{Bun}_{G}\right)$ becomes a module category over $\mathrm{QCoh}\left(\operatorname{LocSys}_{H}^{\mathcal{G}_{Z}}\right)$.

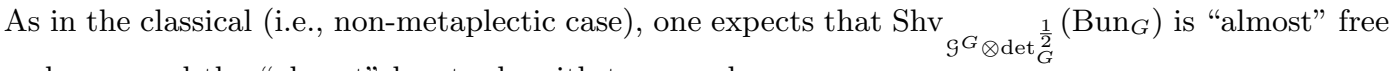
of rank one, and the "almost" has to do with temperedness.

More precisely, one expects that the metaplectic geometric Satake functor (8.3) extends to a derived metaplectic geometric Satake equivalence, generalizing [Ga5, Sects. 4.6 and 4.7], which one can use in order to define the tempered part of $\mathrm{Shv}_{\mathcal{G}^{G} \otimes \operatorname{det}_{G}^{\frac{1}{2}}}\left(\operatorname{Bun}_{G}\right)$, as in [AG, Sect. 12.8].

Now, one expects that the tempered subcategory of $\mathrm{Shv}_{{ }_{g^{G}} \otimes \operatorname{det}_{G}^{\frac{1}{2}}}\left(\mathrm{Bun}_{G}\right)$ is free of rank one as a module over QCoh $\left(\operatorname{LocSys}_{H}^{\mathcal{G}}\right)$.

However, it is not clear whether this module admits a distinguished generator.

8.6.6. Furthermore, one expects that the entire $\mathrm{Shv}_{{ }_{\mathcal{F}^{G}} \otimes \operatorname{det}_{G}^{\frac{1}{2}}}\left(\operatorname{Bun}_{G}\right)$ is non-canonically equivalent to the category IndCoh nilp $\left(\operatorname{LocSys}_{H}^{\mathcal{G} Z}\right)$, where we refer the reader to [AG, Sect. 11.1] for the IndCoh nilp notation.

8.6.7. When $G=T$ is a torus, we have

$$
\left.\operatorname{IndCoh} \operatorname{nilp}_{\text {nocSys }}{ }_{H}^{\mathcal{G}_{Z}}\right)=\mathrm{QCoh}\left(\operatorname{LocSys}_{H}^{\mathcal{G}_{Z}}\right) .
$$

In particular, the equivalence of Sect. 8.6.6 says that for each $\sigma \in \operatorname{LocSys}_{H}^{\mathcal{G}_{Z}}$, the corresponding category of Hecke eigensheaves is non-canonically equivalent to Vect. This equivalence can be made explicit as follows:

A point $\sigma \in \operatorname{LocSys}_{H}^{\mathcal{G}_{Z}}$ gives rise to a trivialization of the pullback of the gerbe $\mathcal{G}^{T}$ from Bun ${ }_{T}$ to Bun $_{T^{\sharp}}$. Hence, it gives rise to a central extension

$$
1 \rightarrow \mathbb{G}_{m} \rightarrow \text { Heis }_{\sigma} \rightarrow \text { Bun }_{\text {ker }(T \sharp T)} \rightarrow 1,
$$

which is easily seen to be of Heinsenberg type, i.e., corresponding to a non-degenerate symplectic form on $\operatorname{ker}\left(T^{\sharp} \rightarrow T\right)$ with values in $\mathbb{G}_{m}$.

The category of Hecke eigensheaves with respect to $\sigma$ is canonically equivalent to

$$
\left(\operatorname{Shv}_{\mathcal{G}^{T}}\left(\operatorname{Bun}_{T}\right)\right)^{\operatorname{Bun}_{T}^{\sharp},}
$$

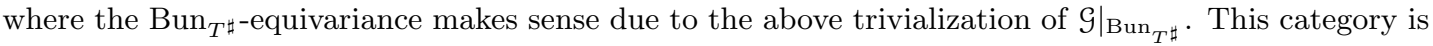
canonically equivalent to the category of representations of Heis $\sigma$, on which $\mathbb{G}_{m}$ acts by the standard character.

Since Heis ${ }_{\sigma}$ is of Heinsenberg type, the above category is non-canonically equivalent to Vect.

8.6.8. At the moment, we do not have a conjecture as to how to explicitly describe the category of Hecke eigensheaves in the tempered subcategory of $\mathrm{Shv}_{\mathcal{G}^{G} \otimes \operatorname{det}_{G}^{\frac{1}{2}}}\left(\operatorname{Bun}_{G}\right)$ with respect to a given $\sigma$ for a general reductive $G$.

\section{REFERENCES}

[AG] D. Arinkin and D. Gaitsgory, Singular support of coherent sheaves, and the geometric Langlands conjecture, Selecta Math. N.S. 21 (2015), 1-199.

[BD1] A. Beilinson and V. Drinfeld, Chiral algebras, AMS Colloquium Publications 51, AMS (2004)

[BD2] A. Beilinson and V. Drinfeld, Quantization of Hitchin's integrable system and Hecke eigensheaves, available at http://www.math.harvard.edu/ gaitsgde/grad2009/

[BrDe] J.-L. Brylinski and P. Deligne, Central extensions of reductive groups by $K_{2}$, Publ. Math. Inst. Hautes E?tudes Sci., 94 (2001), 5-85.

[DrSi] V. Drinfeld and C. Simpson, B-structures on G-bundles and local triviality, MRL 2 (1995), 823-829.

[FL] M. Finkelberg and S. Lysenko, Twisted geometric Satake equivalence, J. Inst. Math. Jussieu 9 (2010), $719-739$. 
[Ga1] D. Gaitsgory, Sheaves of categories and the notion of 1-affineness, in: Stacks and categories in geometry, topology, and algebra, 127-225, Contemp. Math. 643, AMS.

[Ga2] D. Gaitsgory, The Atiyah-Bott formula for the cohomology of the moduli space of bundles on a curve, arXiv: 1505.02331.

[Ga3] D. Gaitsgory, Contractibility of the space of rational maps, Invent. Math. 191 (2013), 91-196.

[Ga4] D. Gaitsgory, Quantum Langlands Correspondence, arXiv:1601.05279.

[Ga5] D. Gaitsgory, Outline of the proof of the geometric Langlands conjecture for GL(2), Astérisque 370 (2015), $1-112$.

[GL1] D. Gaitsgory and J. Lurie, Weil's conjecture for function fields-I, available from http://www.math.harvard.edu/ lurie.

[GL2] D. Gaitsgory and J. Lurie, Weil's conjecture for function fields, available from http://www.math.harvard.edu/ lurie.

[GR1] D. Gaitsgory and N. Rozenblyum, D-modules and crystals, Pure Appl. Math. Q. 10 (2014), $57-154$.

[GR2] D. Gaitsgory and N. Rozenblyum, A study in derived algebraic geometry Volume 1, AMS (2017).

[Lu1] J. Lurie, Higher Topos Theory, Princeton University Press (2009).

[Lu2] J. Lurie, Higher Algebra, available from http://www.math.harvard.edu/ lurie.

[Lus] G. Lusztig, Introduction to quantum groups, Progress in Mathematics 110, Birkhäuser Boston (1993).

[Re] R. Reich, Twisted geometric Satake equivalence via gerbes on the factorizable Grassmannian, Represent. Theory 16 (2012), 345-449.

[Ro] N. Rozenblyum, The local-to-gobal principle, Talk IV.1 at the workshop "Towards the proof of the geometric Langlands conjecture", available from https://sites.google.com/site/geometriclanglands2014/notes

[Sou] C. Soulé, K-théorie des anneaux dentiers de corps de nombres et cohomologie étale, Inv. Math. 55 (1979), 251-295.

[Ras1] S. Raskin, Chiral categories, available from http://math.mit.edu/ sraskin/

[Ras2] S. Raskin, Chiral principal series categories I: finite-dimensional calculations, available from http://math.mit.edu/ sraskin/

[We] M. Weissman, L-groups and parameters for covering groups, arXiv:1507.0104. 